Document downloaded from:

http://hdl.handle.net/10251/57541

This paper must be cited as:

Achary, S.N.; Errandonea, D.; Muñoz, A.; Rodríguez Hernández, P.;Manjón, F.J.; Kishna, P. S. R.; Patwe, S. J.; Grover, V. ; Tyagi, A. (2013). Experimental and theoretical investigations on the polymorphism and metastability of BiPO4. Dalton Transactions. 42:14999-15015. doi:10.1039/c3dt51823j.

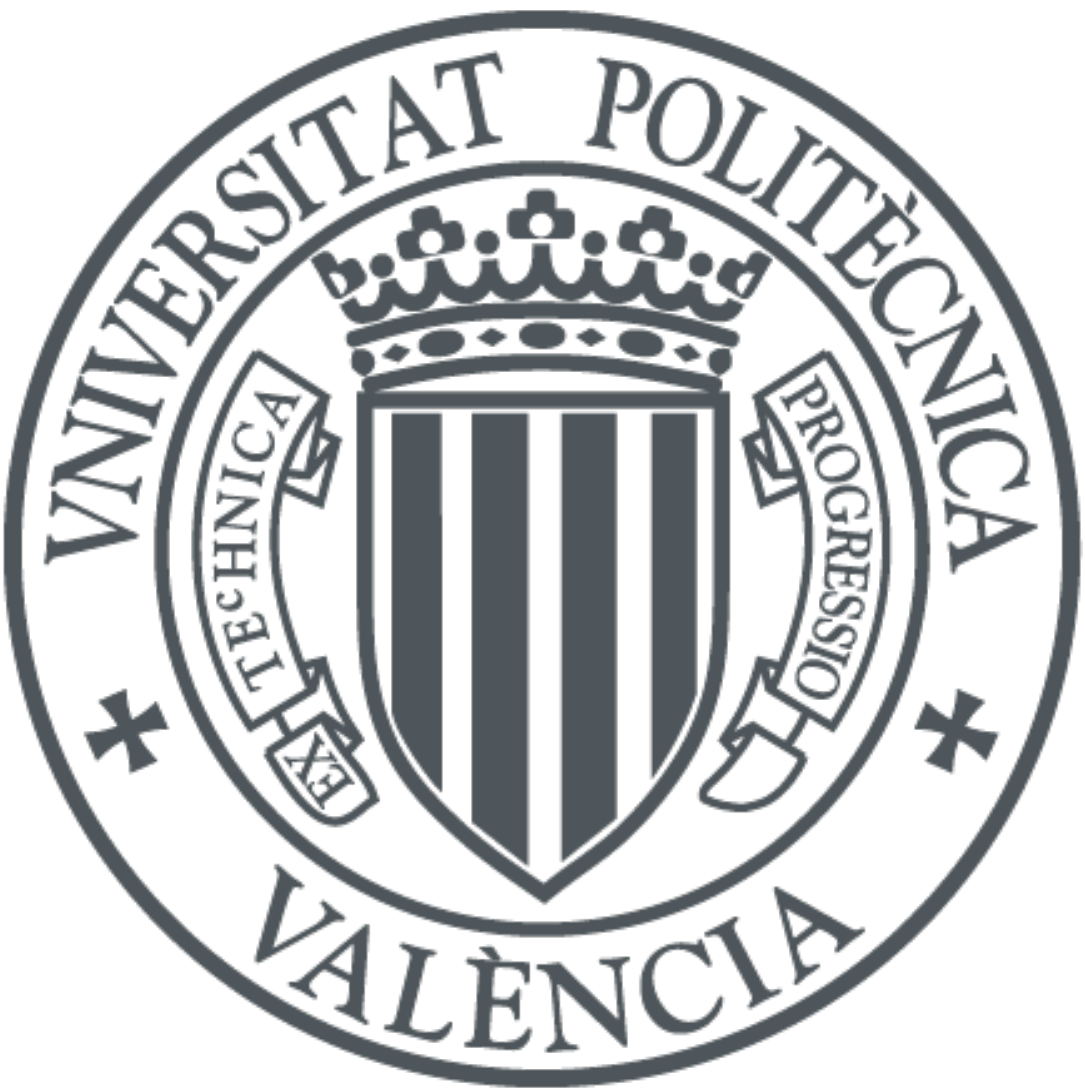

The final publication is available at

http://dx.doi.org/10.1039/c3dt51823j

Copyright Royal Society of Chemistry

Additional Information 


\title{
Experimental and theoretical investigations on polymorphism and metastability of $\mathrm{BiPO}_{4}$
}

\author{
S. N. Achary ${ }^{1 *}$, D. Errandonea ${ }^{2}$, A. Muñoz ${ }^{3}$, P. Rodríguez-Hernández ${ }^{3}$, F. J. Manjón ${ }^{4}$, \\ P. S. R. Krishna ${ }^{5}$, S. J. Patwe ${ }^{1}$, V. Grover ${ }^{1}$ and A. K. Tyagi ${ }^{1}$ \\ ${ }^{1}$ Chemistry Division, Bhabha Atomic Research Centre, Mumbai 400085, India \\ ${ }^{2}$ MALTA Consolider Team, Departamento de Física Aplicada-ICMUV, Universitad de Valencia, \\ Edificio de Investigación, c/Dr. Moliner 50, Burjassot, 46100 Valencia, Spain \\ ${ }^{3}$ Departamento de Física Fundamental II, Instituto de Materiales y Nanotecnología, MALTA \\ Consolider Team, Universidad de La Laguna, La Laguna 38205, Tenerife, Spain \\ ${ }^{4}$ Instituto de Diseño para la Fabricación y Producción Automatizada, MALTA Consolider \\ Team, Universitat Politècnica de València, 46022 València, Spain \\ ${ }^{5}$ Solid State Physics Division, Bhabha Atomic Research Centre, Mumbai 400085, India
}

\begin{abstract}
In this communication we report the metastabilty and energetic of phase transitions of three different polymorphs of $\mathrm{BiPO}_{4}$, namely trigonal (Phase-I, space group $\mathrm{P}_{1} 21$ ), monoclinic monazite-type (Phase-II, space group $\mathrm{P}_{1} / \mathrm{n}$ ) and $\mathrm{SbPO}_{4}$-type monoclinic (Phase-III, space group $\mathrm{P} 2{ }_{1} / \mathrm{m}$ ) from ambient and non ambient temperature powder XRD and neutron diffraction studies as well as $a b$ initio Density Functional Theory (DFT) calculations. The symmetry ambiguity between $\mathrm{P} 2_{1}$ and $\mathrm{P} 2_{1} / \mathrm{m}$ of the high temperature polymorph of $\mathrm{BiPO}_{4}$ has been resolved by neutron diffraction study. The structure and vibrational properties of these polymorphs of the three polymorphs have also been reported in detail. The total energy calculations have been used to understand the experimentally observed metastable behavior of trigonal and monazite-type $\mathrm{BiPO}_{4}$. Interestingly, all the three phases were found to coexist after heating a single phasic trigonal $\mathrm{BiPO}_{4}$ to $773 \mathrm{~K}$. The irreversible natures of these phase transitions have been explained on the concepts of interplay of structural distortion, molar volume and total energy.
\end{abstract}

Address for Correspondence

Dr. S. N. Achary,

Chemistry Division, Bhabha Atomic Research Centre, Mumbai - 400 085, India

Email: sachary@barc.gov.in; acharysn@rediffmail.com

Phone: 0091-22-2559 2328; Fax : 0091-22-2550 5151 
Lattice-dynamic calculations of the phonon modes were performed at the zone center $(\Gamma$ point) of the BZ. We used a direct force-constant approach (or supercell method) ${ }^{43}$ as it is conceptually simple. These calculations provide information about the symmetry of the modes and their polarization vectors which allowed us to identify the irreducible representations and the character of the phonon modes at the $\Gamma$ point. However, this method did not include the contribution from dipole-dipole interactions resulting the LO-TO splitting of infrared-active modes, due to the non-analyticity of this term at the $\Gamma$ point.

\section{Results and discussion}

\section{1. $X$-ray and neutron diffraction studies at ambient conditions}

The powder XRD patterns of the three phases of $\mathrm{BiPO}_{4}$, namely BiPO4-I, II and III (shown in Fig. 1) are in agreement with trigonal, monoclinic monazite-type (JCPDS-PDF-800209) and monoclinic high-temperature $\mathrm{SbPO}_{4}$ type (JCPDS-PDF-77-2208), respectively. The complete transformation from one phase to other and retention of the transformed phases is a unique example observed in $\mathrm{BiPO}_{4}$ compared to other orthophosphates of trivalent cations. It has been argued that that the water molecules in $\mathrm{BiPO}_{4}$ play a crucial role in stabilizing the trigonal structure of $\mathrm{BiPO}_{4}$-I. ${ }^{32}$ Such hydrated hexagonal forms have been reported for almost all the trivalent rare-earth cations and upon dehydration the structure is destabilized and transforms to other structure types. ${ }^{44}$ The comparable coherent neutron scattering lengths of various atoms and negative scattering length of the hydrogen $\left(b_{O}=0.580 \times 10^{-12} \mathrm{~cm}, b_{B i}=0.853 \times 10^{-12} \mathrm{~cm}, b_{P}=\right.$ $0.513 \times 10^{-12} \mathrm{~cm}, \mathrm{~b}_{\mathrm{H}}=-0.374 \times 10^{-12} \mathrm{~cm}$ ) were helpful to obtain accurate structural parameters by neutron diffraction compared to the x-ray diffraction. The detailed structural parameters of the three phases were obtained by Rietveld refinement of the powder neutron diffraction data. The initial structural models for the for the Rietveld refinements were taken from the structural data reported by Romero et al. ${ }^{32}$ (for trigonal $\mathrm{BiPO}_{4}$-I and monazite-type $\mathrm{BiPO}_{4}-\mathrm{II}$ ) and Masse and Duriff ${ }^{31}$ (for high-temperature $\mathrm{BiPO}_{4}$-III). Diffractometer zero and sample displacements parameters were taken from the neutron diffraction pattern of standard $\mathrm{Yb}_{2} \mathrm{O}_{3}$. The peaks of the neutron diffraction patterns were computed by using Pseudo-Voigt profile function. The backgrounds of the patterns were fitted with shifted Chebyschev polynomial functions. It can be 
evident. This observation is supported by first principle total energy calculations explained later in this manuscript. The different trends in variation of unit cell parameters of $\mathrm{BiPO}_{4}-\mathrm{I}$ are reflected in the variation of unit cell volume (Fig. 7). The variation of molar volume (V/Z) of $\mathrm{BiPO}_{4}$-I with temperature shows feeble decreasing trend up to $473 \mathrm{~K}$. This initial decreasing trend can be assigned to the contraction of the lattice due to the loss of water molecules from the hexagonal channel in the lattice. A subtle positive expansion behavior in between 473 and $673 \mathrm{~K}$ can be attributed thermal expansion behavior of anhydrous trigonal $\mathrm{BiPO}_{4}$ (Phase-I). In a condition, the trigonal, monazite-type and $\mathrm{SbPO}_{4}$-type phase can exist with stoichiometry as $\mathrm{BiPO}_{4}$ and thus they can be regarded as polymorphic transition.

Detailed analyses of the refined structural parameters of the different polymorphs suggest that the $\mathrm{PO}_{4}$ tetrahedra are regular in the monazite-type $\left(\mathrm{BiPO}_{4}\right.$-II) and $\mathrm{SbPO}_{4}$-type $\left(\mathrm{BiPO}_{4}\right.$-III) phases compared to those the trigonal phase. The polyhedral characters of the three phases were calculated by IVTON ${ }^{52,53}$ and they are given in Table 4. It can be mentioned here that the $\mathrm{PO}_{4}$ tetrahedra in trigonal phase do not act like a rigid unit as commonly observed in most phosphates. The average bond lengths of $\mathrm{P}-\mathrm{O}$ of trigonal phase are also longer compared to other two phases. Similar analyses of coordination polyhedra around $\mathrm{Bi}$ in the three phases indicate that the $\mathrm{BiO}_{8}$ or $\mathrm{BiO}_{9}$ units of the $\mathrm{BiPO}_{4}$ phases are distorted and asymmetric. Further the volume distortion ${ }^{53}$ (relative difference between the volume of polyhedra and volume of ideal polyhedra expected for a same radius of circumscribed sphere) and eccentricity (deviation of central atom position from the ideal centre of the coordination polyhedron) of the $\mathrm{BiO}_{\mathrm{n}}$ polyhedra are larger in trigonal phase compared to the other two phases. The details of polyhedral analyses of the high temperature structures are given in Electronic Supplementary Information (ESI-I) ${ }^{\dagger}$. The larger eccentricity and asymmetry of the trigonal phase is minimized by the additional bonding interaction from the $\mathrm{H}_{2} \mathrm{O}$ molecules in the lattice. It is observed that the average $\mathrm{Bi}-\mathrm{O}$ bond lengths and distortion in $\mathrm{BiO}_{8}$ polyhedra increase with the increase in temperature. The increasing trend is significant for trigonal (I) and monazite (II) type phases compared to the $\mathrm{SbPO}_{4}$ type phase (III). This result suggests that the $\mathrm{SbPO}_{4}$ type phase is the stable phase for $\mathrm{BiPO}_{4}$ at ambient condition. The relatively expanded lattice can be attributed to the higher stability of $\mathrm{SbPO}_{4}$-type phase. In the next section we further study the stability of these phases by means of $a b$-initio theoretical calculations. 


\section{Conclusions}

In summary, the detailed analyses of the powder X-ray and neutron diffraction data as well as IR and Raman studies on three polymorphs of $\mathrm{BiPO}_{4}$ revealed accurate structural parameters and local distortions parameters around $\mathrm{Bi}^{3+}$ and $\mathrm{P}^{5+}$. Further the structural parameters and vibrational frequencies were supported by $a b$ initio calculations based on DFT. The irreversible trigonal-monazite- $\mathrm{SbPO}_{4}$-type structural transitions are explained from the molar volume and total energy of the three phases. Highly distorted structural arrangement around $\mathrm{Bi}^{3+}$ and $\mathrm{P}^{5+}$ has a very limited stability and is only stabilized by water molecules in the trigonal phase. It is revealed that structural transition from trigonal to monazite or $\mathrm{SbPO}_{4}$ type structures is accompanied by a significant reduction in volume. DFT calculations revealed that the monazite and $\mathrm{SbPO}_{4}$-type $\mathrm{BiPO}_{4}$ have almost similar total energy and hence a competing stability. Further it is observed that monazite type phase shows larger lattice thermal expansion compared to the $\mathrm{SbPO}_{4}$-type phase and thus it is truly a metastable phase.

\section{Acknowledgements}

This study was supported by the Spanish government MEC under Grants No: MAT2010-21270C04-01/04, by MALTA Consolider Ingenio 2010 project (CSD2007-00045), and by the Vicerrectorado de Investigación y Desarrollo of the Universidad Politécnica de Valencia (UPV2011-0914 PAID-05-11 and UPV2011-0966 PAID-06-11). S.N.A. acknowledges the support provided by Universitat de Valencia during his visit to it. A.M. and P.R-H. acknowledge computing time provided by Red Española de Supercomputación (RES) and MALTA-Cluster.

†Electronic Supplementary Information (ESI) available: [(ESI-1). Further details of crystal structural data of the BiPO4-I, II and III can be obtained from the Fachinformationszentrum Karlsruhe, Abt. PROKA, 76344 Eggenstein-Leo-poldshafen, Germany (fax +49-7247-808-666; E-mail: crysdata@fiz-karlsruhe.de) on quoting the depository numbers CSD 426230 to 426255 (26 structures)]. See DOI: 10.1039/b000000x 


\section{Tables}

Table 1: Refined unit cell parameters for different polymorphs of $\mathrm{BiPO}_{4}$ at ambient temperature (300K). (Calculated unit cell parameters are also included for comparison)

Table-2: Refined and calculated structural parameters of $\mathrm{BiPO}_{4}$ at $300 \mathrm{~K}((\mathrm{a})$. Trigonal (I), (b). Monazite-type (II) and (c). SbPO 4 -type (III)).

Table 3: The typical bond lengths and polyhedral parameters in different phases of $\mathrm{BiPO}_{4}$.

Table 4: Calculated and observed Infrared modes of different $\mathrm{BiPO}_{4}$ phases. ((a). Trigonal (I), (b). Monazite-type (II) and (c). SbPO4-type (III).)

Table 5: Calculated and observed Raman modes of $\mathrm{BiPO}_{4}$ phases. ((a). Trigonal (I), (b). Monazite-type (II) and (c). SbPO 4 -type (III)). 
Table 3: The typical bond lengths and polyhedral parameters in different phases of $\mathrm{BiPO}_{4}$.

\begin{tabular}{|c|c|c|c|c|c|}
\hline \multicolumn{2}{|c|}{$\mathrm{BIPO}_{4}-\mathrm{I}$ (Trigonal) } & \multicolumn{2}{|c|}{$\mathrm{BIPO}_{4}$-II (Monazite-type) } & \multicolumn{2}{|c|}{$\mathrm{BIPO}_{4}$-III $\left(\mathrm{SbPO}_{4}\right.$-type $)$} \\
\hline $\mathrm{Bi}-\mathrm{O} 1$ & $2.817(8)(\AA)$ & $\mathrm{Bi}-\mathrm{O} 1$ & $2.580(6)(\AA)$ & $\mathrm{Bi}-\mathrm{O} 1$ & $2.149(4)(\AA)$ \\
\hline $\mathrm{Bi}-\mathrm{O} 1$ & $2.296(8)$ & $\mathrm{Bi}-\mathrm{O} 1$ & $2.492(5)$ & $\mathrm{Bi}-\mathrm{O} 1$ & $2.880(4)$ \\
\hline $\mathrm{Bi}-\mathrm{O} 1$ & $2.817(7)$ & $\mathrm{Bi}-\mathrm{O} 2$ & $3.020(5)$ & $\mathrm{Bi}-\mathrm{O} 2$ & $2.896(4)$ \\
\hline $\mathrm{Bi}-\mathrm{O} 1$ & $2.296(12)$ & $\mathrm{Bi}-\mathrm{O} 2$ & $2.399(6)$ & $\mathrm{Bi}-\mathrm{O} 2$ & $2.330(3)$ \\
\hline $\mathrm{Bi}-\mathrm{O} 2$ & $2.378(7)$ & $\mathrm{Bi}-\mathrm{O} 2$ & $2.700(5)$ & $\mathrm{Bi}-\mathrm{O} 3$ & $2.357(3)$ \\
\hline $\mathrm{Bi} 1-\mathrm{O} 2$ & $2.490(9)$ & $\mathrm{Bi}-\mathrm{O} 3$ & $2.361(6)$ & $\mathrm{Bi}-\mathrm{O} 3$ & $2.526(3)$ \\
\hline $\mathrm{Bi}-\mathrm{O} 2$ & $2.490(7)$ & $\mathrm{Bi}-\mathrm{O} 3$ & $2.394(6)$ & $\mathrm{Bi}-\mathrm{O} 3$ & $2.526(3)$ \\
\hline \multirow[t]{2}{*}{$\mathrm{Bi}-\mathrm{O} 2$} & $2.378(8)$ & $\mathrm{Bi}-\mathrm{O} 4$ & $2.469(5))$ & $\mathrm{Bi}-\mathrm{O} 3$ & $2.357(3)$ \\
\hline & & $\mathrm{Bi}-\mathrm{O} 4$ & $2.426(6)$ & & \\
\hline CN: & $6+2$ & $\mathrm{CN}$ & $8+1$ & $\mathrm{CN}$ & 8 \\
\hline$<\mathrm{Bi}-\mathrm{O}>$ & 2.495(3) ( $)$ & $<\mathrm{Bi}-\mathrm{O}>_{9}$ & $2.538(2)(\AA)$ & $<\mathrm{Bi}-\mathrm{O}>$ & $2.503(1)(\AA)$ \\
\hline \multirow[t]{3}{*}{ Distt. } & $62.976 \times 10^{-4}$ & Distt. & $60.598 \times 10^{-4}$ & Distt. & $98.915 \times 10^{-4}$ \\
\hline & & $<\mathrm{Bi}-\mathrm{O}>_{8}$ & $2.478(2)(\AA)$ & & \\
\hline & & Distt. & $18.238 \times 10^{-4}$ & & \\
\hline $\mathrm{V}_{\mathrm{BiO} 8}\left(\AA^{3}\right)$ & 29.06 & $\mathrm{~V}_{\mathrm{BiO} 8}\left(\AA^{3}\right)$ & 26.26 & $\mathrm{~V}_{\mathrm{BiO} 8}\left(\AA^{3}\right)$ & 27.28 \\
\hline Distt. & 0.1491 & Distt. & 0.0684 & Distt. & 0.0819 \\
\hline Sphericity & 0.844 & Sphericity & 0.941 & Sphericity & 0.910 \\
\hline \multirow[t]{5}{*}{ Eccentricity } & 0.346 & Eccentricity & 0.196 & Eccentricity & 0.449 \\
\hline & & $\mathrm{V}_{\mathrm{BiO}}\left(\AA^{3}\right)$ & 31.95 & & \\
\hline & & Distt. & 0.0557 & & \\
\hline & & Sphericity & 0.853 & & \\
\hline & & Eccentricity & 0.308 & & \\
\hline $\mathrm{P}-\mathrm{O} 1$ & $1.523(12)(\AA)$ & $\mathrm{P}-\mathrm{O} 1$ & $1.550(7)(\AA)$ & P - O1 & $1.554(4)(\AA)$ \\
\hline P - O1 & $1.523(9)$ & $\mathrm{P}-\mathrm{O} 2$ & $1.567(7)$ & $\mathrm{P}-\mathrm{O} 2$ & $1.552(4)$ \\
\hline $\mathrm{P}-\mathrm{O} 2$ & 1.592(9) & $\mathrm{P}-\mathrm{O} 3$ & $1.539(6)$ & $\mathrm{P}-\mathrm{O} 3$ & $1.526(3)$ \\
\hline $\mathrm{P}-\mathrm{O} 2$ & $1.592(10)$ & $\mathrm{P}-\mathrm{O} 4$ & $1.509(8)$ & $\mathrm{P}-\mathrm{O} 3$ & $1.526(3)$ \\
\hline$<\mathrm{P}-\mathrm{O}>_{4}$ & $1.558(5)(\AA)$ & $<\mathrm{P}-\mathrm{O}>_{4}$ & $1.541(4)(\AA)$ & $<\mathrm{P}-\mathrm{O}>$ & $1.540(2)(\AA)$ \\
\hline Distt. & $4.895 \times 10^{-4}$ & Distt. & $1.839 \times 10^{-4}$ & Distt. & $0.757 \times 10^{-4}$ \\
\hline $\mathrm{V}_{\mathrm{PO} 4}\left(\AA^{3}\right)$ & 1.923 & $\mathrm{~V}_{\mathrm{PO} 4}\left(\AA^{3}\right)$ & 1.862 & $\mathrm{~V}_{\mathrm{PO} 4}\left(\AA^{3}\right)$ & 1.861 \\
\hline Distt. & 0.0128 & Distt. & 0.0094 & Distt. & 0.0070 \\
\hline Sphericity & 1 & Sphericity & 1 & Sphericity & 1 \\
\hline Eccentricity & 0.042 & Eccentricity & 0.066 & Eccentricity & 0.116 \\
\hline
\end{tabular}


(b). Monazite-type $\left(\mathrm{BiPO}_{4}-\mathrm{II}\right)$

\begin{tabular}{|c|c|c|c|c|c|}
\hline Mode & $\operatorname{LDA} \omega\left(\mathrm{cm}^{-1}\right)$ & Mode & PBESol $\omega\left(\mathrm{cm}^{-1}\right)$ & Mode & Exper $\omega\left(\mathrm{cm}^{-1}\right)$ \\
\hline $\operatorname{Ag}(\mathrm{R})$ & 64.378 & $\operatorname{Ag}(\mathrm{R})$ & 58.209 & $\mathrm{Ag}$ & 50,9 \\
\hline $\operatorname{Ag}(R)$ & 74.285 & $\operatorname{Ag}(\mathrm{R})$ & 68.383 & $\mathrm{Ag}$ & 59,8 \\
\hline $\mathrm{Bg}(\mathrm{R})$ & 75.286 & $\mathrm{Bg}(\mathrm{R})$ & 73.287 & $\mathrm{Bg}$ & 69,9 \\
\hline $\mathrm{Bg}(\mathrm{R})$ & 93.699 & $\mathrm{Bg}(\mathrm{R})$ & 89.332 & $\mathrm{Bg}$ & 89,6 \\
\hline $\operatorname{Ag}(R)$ & 97.335 & $\operatorname{Ag}(\mathrm{R})$ & 95.337 & Ag & 97,3 \\
\hline \multirow[t]{2}{*}{$\mathrm{Bg}(\mathrm{R})$} & 106.908 & $\mathrm{Bg}(\mathrm{R})$ & 102.642 & $\mathrm{Bg}$ & 108,6 \\
\hline & & $\operatorname{Ag}(R)$ & 132.697 & $\mathrm{Bg}$ & 131,1 \\
\hline $\mathrm{Bg}(\mathrm{R})$ & 134.561 & $\mathrm{Bg}(\mathrm{R})$ & 134.732 & $\mathrm{Bg}$ & 135,5 \\
\hline $\operatorname{Ag}(R)$ & 138.597 & & & & \\
\hline $\operatorname{Ag}(\mathrm{R})$ & 166.950 & $\operatorname{Ag}(\mathrm{R})$ & 165.688 & $\mathrm{Ag}$ & 169,9 \\
\hline $\mathrm{Bg}(\mathrm{R})$ & 172.921 & $\mathrm{Bg}(\mathrm{R})$ & 167.790 & $\mathrm{Bg}+\mathrm{Ag}$ & 176,7 \\
\hline $\operatorname{Ag}(R)$ & 172.787 & $\operatorname{Ag}(\mathrm{R})$ & 168.424 & & \\
\hline \multirow[t]{2}{*}{$A g(R)+B g(R)$} & 192.601 & $\operatorname{Ag}(\mathrm{R})$ & 184.002 & $\mathrm{Ag}+\mathrm{Bg}$ & 182,7 \\
\hline & & $\mathrm{Bg}(\mathrm{R})$ & 185.236 & & \\
\hline $\mathrm{Bg}(\mathrm{R})$ & 212.115 & $\mathrm{Bg}(\mathrm{R})$ & 203.883 & $\mathrm{Bg}$ & 207,4 \\
\hline $\mathrm{Bg}(\mathrm{R})$ & 235.931 & $\operatorname{Bg}(\mathrm{R})$ & 226.199 & $\mathrm{Bg}$ & 229,8 \\
\hline $\operatorname{Ag}(\mathrm{R})$ & 240.434 & $\operatorname{Ag}(\mathrm{R})$ & 231.903 & $\mathrm{Ag}$ & 237,1 \\
\hline $\mathrm{Bg}(\mathrm{R})$ & 279.095 & $\mathrm{Bg}(\mathrm{R})$ & 271.332 & $\mathrm{Bg}$ & 272,5 \\
\hline $\mathrm{Ag}(\mathrm{R})$ & 286.133 & $\operatorname{Ag}(\mathrm{R})$ & 278.671 & $\mathrm{Ag}$ & 283,5 \\
\hline $\mathrm{Bg}(\mathrm{R})$ & 375.729 & $\operatorname{Bg}(\mathrm{R})$ & 368.303 & Bg & 388,4 \\
\hline $\operatorname{Ag}(R)$ & 390.439 & $\operatorname{Ag}(\mathrm{R})$ & 384.549 & $\mathrm{Ag}$ & 407,4 \\
\hline $\mathrm{Ag}(\mathrm{R})$ & 445.077 & $\operatorname{Ag}(\mathrm{R})$ & 438.688 & $\mathrm{Ag}$ & 457,0 \\
\hline $\mathrm{Bg}(\mathrm{R})$ & 477.833 & $\operatorname{Bg}(\mathrm{R})$ & 469.945 & $\mathrm{Bg}$ & 463,5 \\
\hline $\mathrm{Ag}(\mathrm{R})$ & 503.051 & $\operatorname{Ag}(\mathrm{R})$ & 497.999 & Ag & 495,9 \\
\hline $\operatorname{Ag}(R)$ & 531.404 & $\operatorname{Ag}(\mathrm{R})$ & 527.220 & $\mathrm{Ag}$ & 522,7 \\
\hline $\mathrm{Bg}(\mathrm{R})$ & 532.638 & $\mathrm{Bg}(\mathrm{R})$ & 527.287 & $\mathrm{Bg}$ & 557,1 \\
\hline $\mathrm{Bg}(\mathrm{R})$ & 547.749 & $\mathrm{Bg}(\mathrm{R})$ & 540.930 & $\mathrm{Bg}$ & 572,6 \\
\hline $\operatorname{Ag}(\mathrm{R})$ & 573.867 & $\operatorname{Ag}(\mathrm{R})$ & 568.083 & $\mathrm{Ag}$ & 598,3 \\
\hline $\mathrm{Bg}(\mathrm{R})$ & 580.405 & $\mathrm{Bg}(\mathrm{R})$ & 573.921 & $\mathrm{Bg}$ & 604,5 \\
\hline $\mathrm{Ag}(\mathrm{R})$ & 933.184 & $\operatorname{Ag}(\mathrm{R})$ & 911.936 & $\mathrm{Ag}+\mathrm{Bg}$ & 926,3 \\
\hline $\mathrm{Bg}(\mathrm{R})$ & 933.685 & $\mathrm{Bg}(\mathrm{R})$ & 915.772 & & \\
\hline $\operatorname{Ag}(\mathrm{R})$ & 954.933 & $\operatorname{Ag}(\mathrm{R})$ & 935.286 & $\mathrm{Ag}$ & 947,8 \\
\hline $\operatorname{Ag}(\mathrm{R})$ & 973.412 & $\operatorname{Ag}(\mathrm{R})$ & 956.502 & $\mathrm{Ag}$ & 969,5 \\
\hline $\mathrm{Bg}(\mathrm{R})$ & 982,552 & $\mathrm{Bg}(\mathrm{R})$ & 962.272 & $\mathrm{Bg}$ & 981,0 \\
\hline $\mathrm{Bg}(\mathrm{R})$ & 1001.799 & $\mathrm{Bg}(\mathrm{R})$ & 985.256 & $\mathrm{Bg}$ & 1021,0 \\
\hline $\operatorname{Ag}(\mathrm{R})$ & 1027.383 & $\operatorname{Ag}(R)$ & 1010.141 & $\mathrm{Ag}$ & 1039,4 \\
\hline $\mathrm{Bg}(\mathrm{R})$ & 1035.389 & $\mathrm{Bg}(\mathrm{R})$ & 1016.612 & $\mathrm{Bg}$ & 1050,2 \\
\hline
\end{tabular}




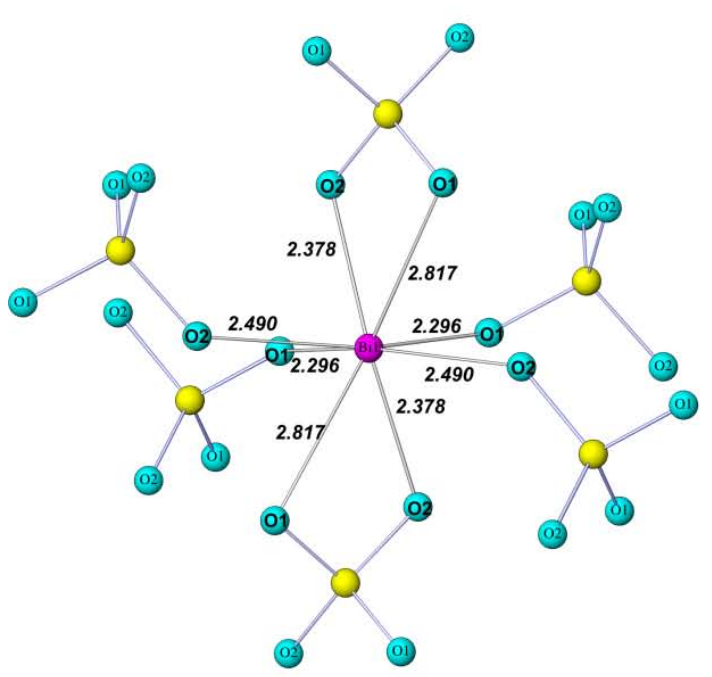

(a)

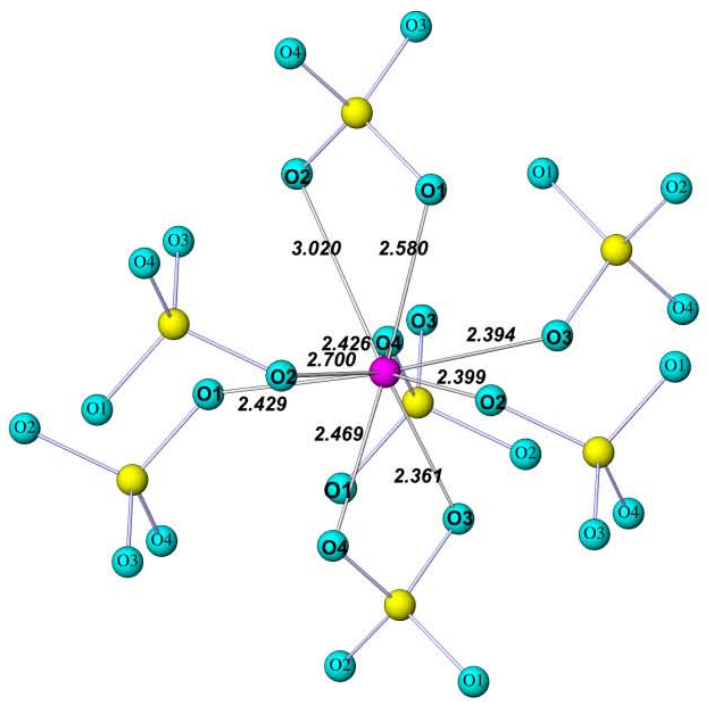

(b)

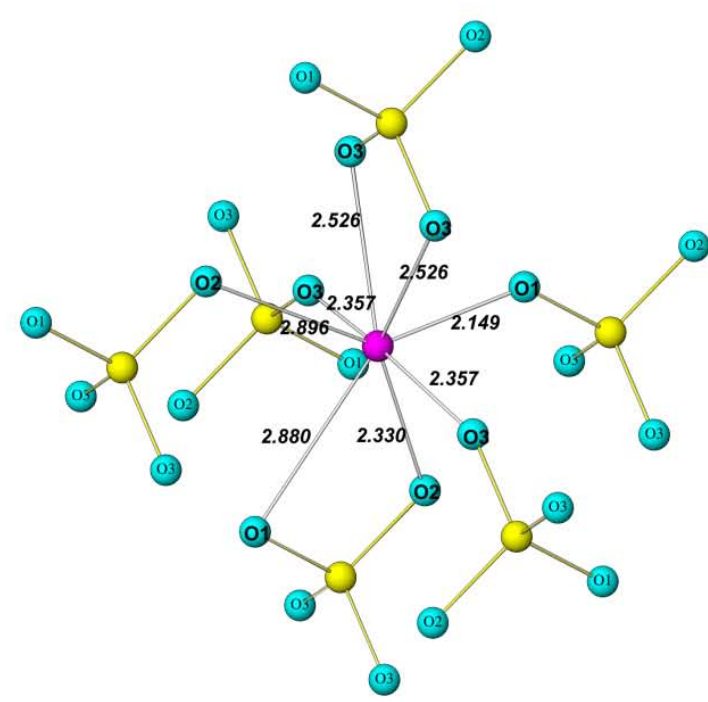

(c)

Fig. 4: Typical coordination around $\mathrm{Bi}^{3+}$ in $\mathrm{BiPO}_{4}$ phases, (a). Trigonal (I), (b). Monazite type (II) and (c). SbPO 4 type (III) 

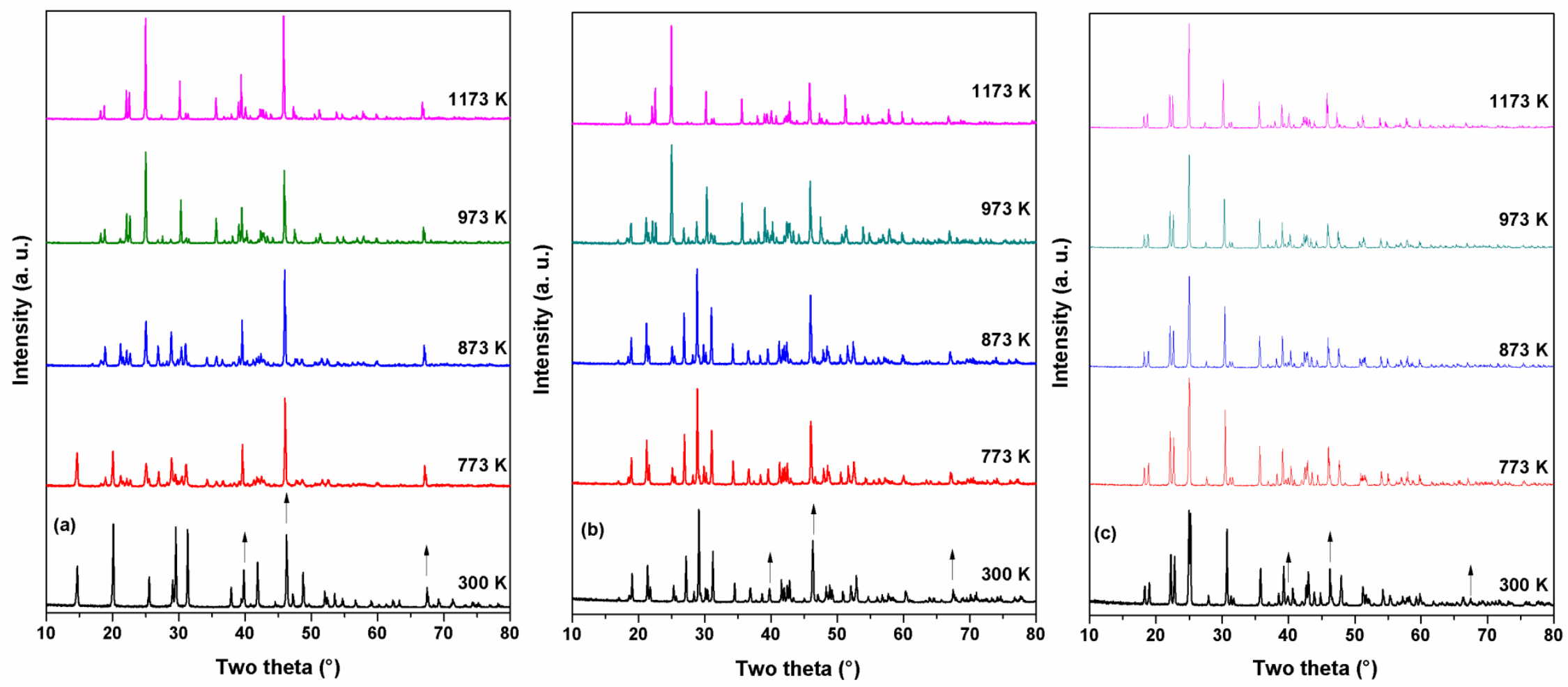

Fig. 5: Powder XRD patterns of $\mathrm{BiPO}_{4}$ phases at representative temperatures. (a). Trigonal (I), (b). Monazite-type (II) and (c). SbPO ${ }_{4-}$ type (III) ( $\uparrow$ indicate platinum sample holder base peaks) 


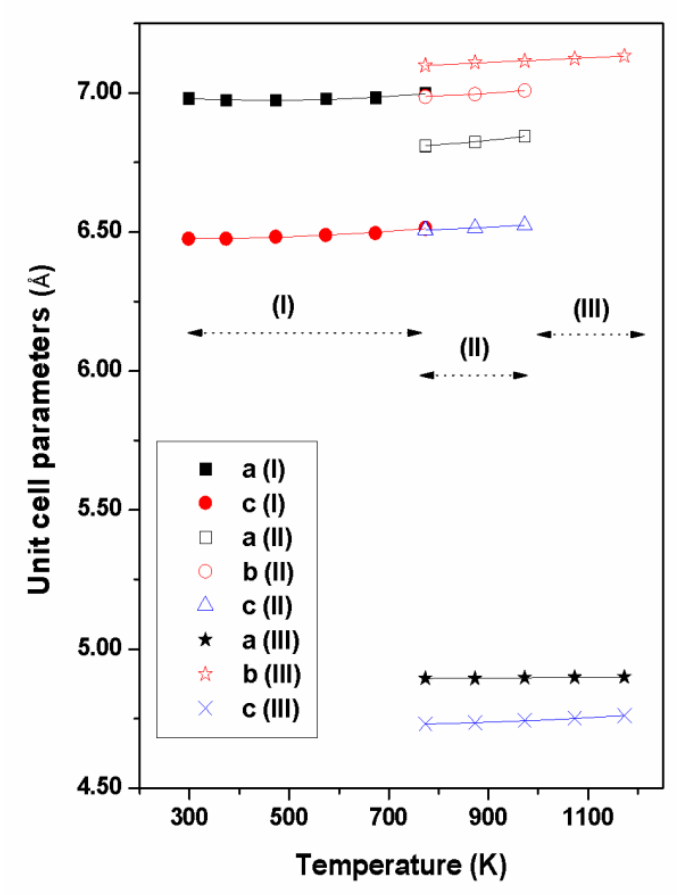

(a)

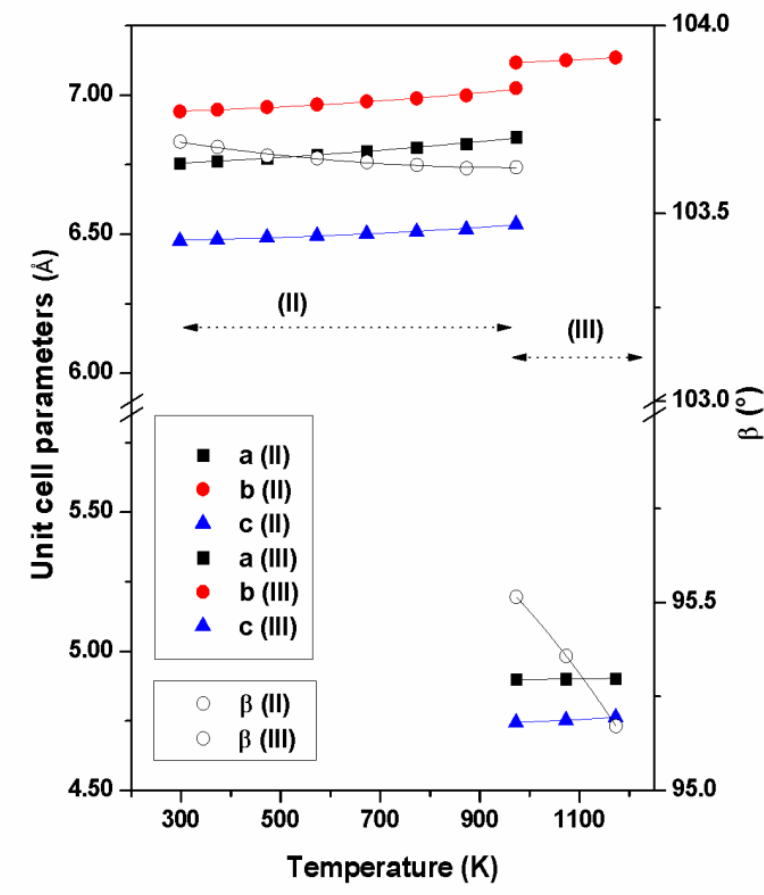

(b)

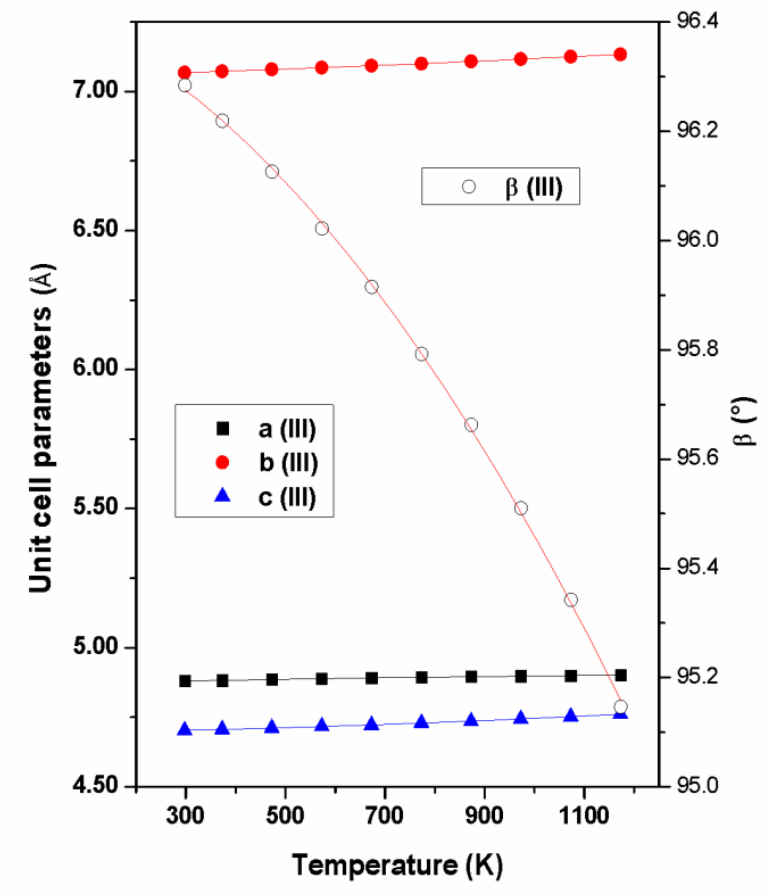

(c)

Fig. 6: Variation of unit cell parameters of $\mathrm{BiPO}_{4}$ with temperature. (a). Trigonal (I), (b). Monazite-type (II) and (c). SbPO 4 -type (III). 


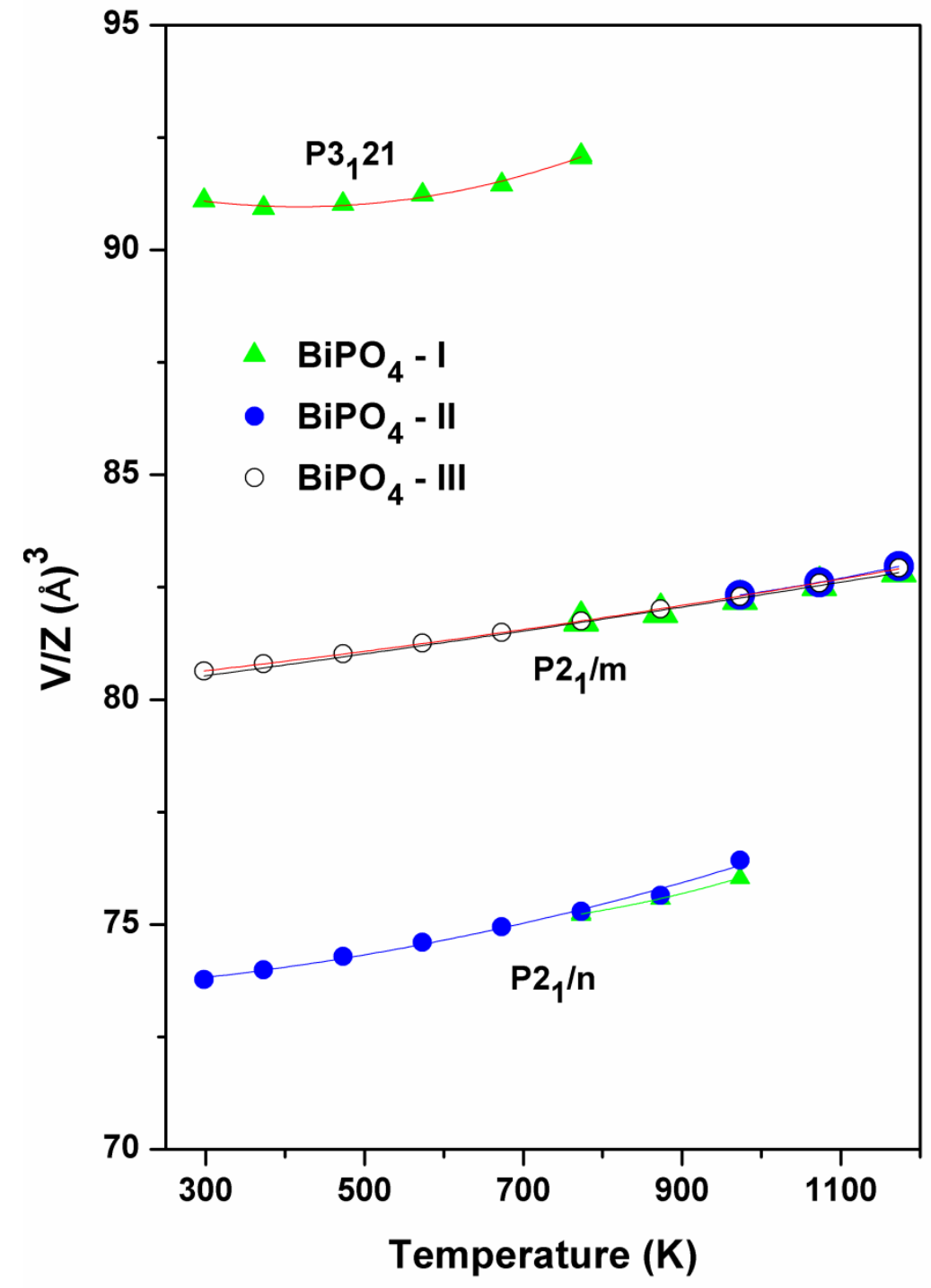

Fig. 7: Variation of molar volume of $\mathrm{BiPO}_{4}$ phases with temperature. 

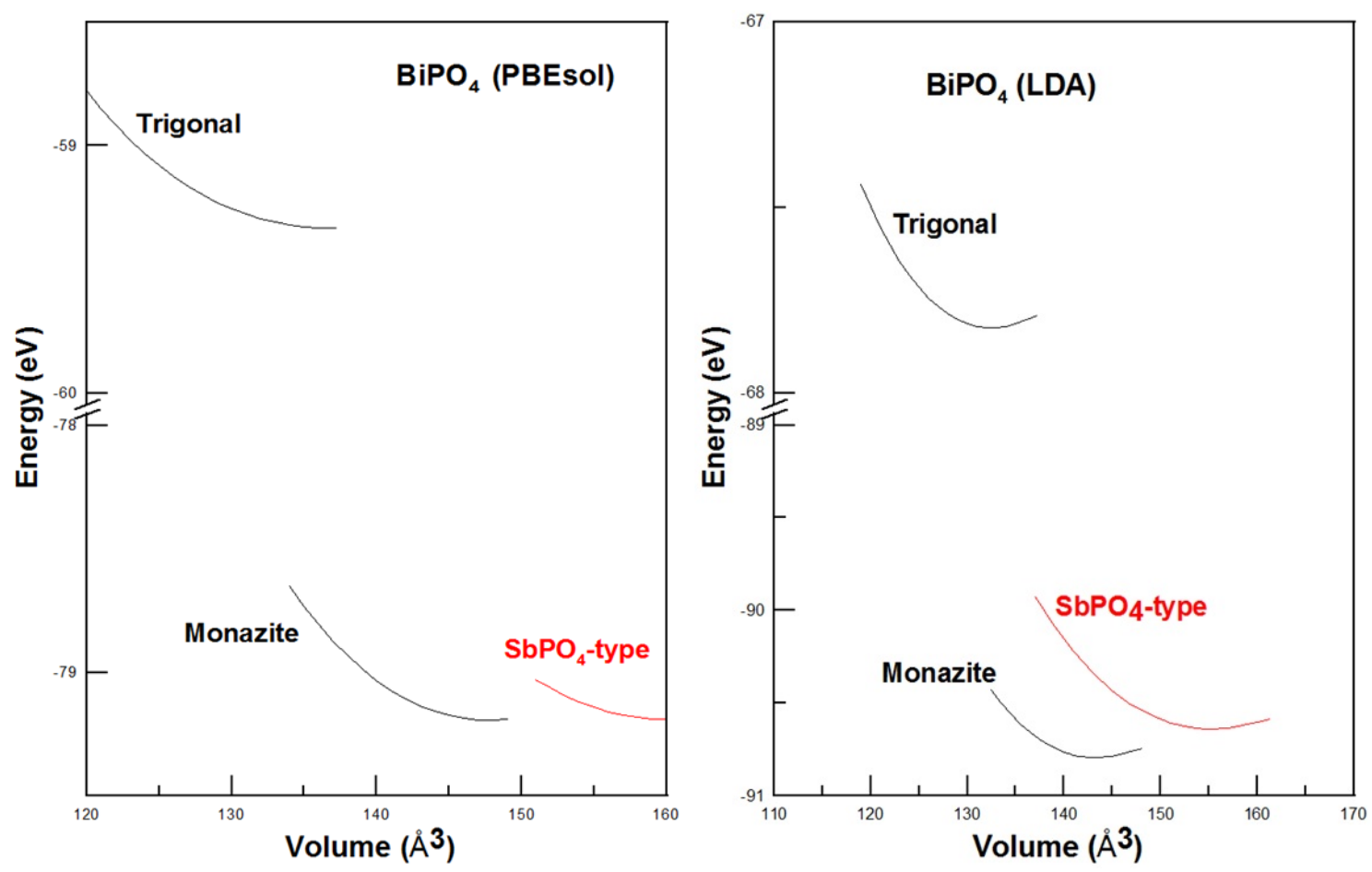

Fig. 8: Total energy as calculated by LDA and PBESol approach. 

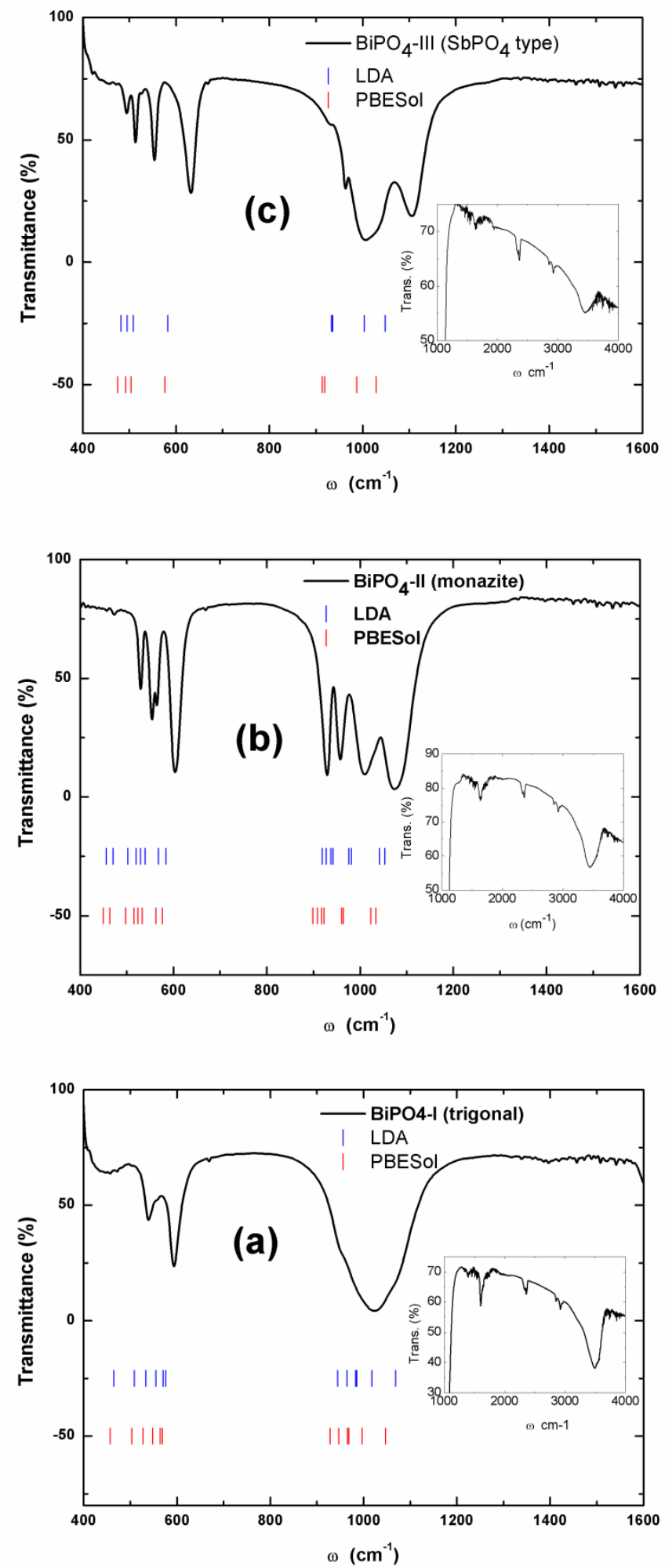

Fig. 9: Typical FTIR spectra of $\mathrm{BiPO}_{4}$ phases. (a). Trigonal (I), (b). Monazite-type (II) and (c). SbPO 4 -type (III). Vertical ticks indicate the calculated frequencies. 

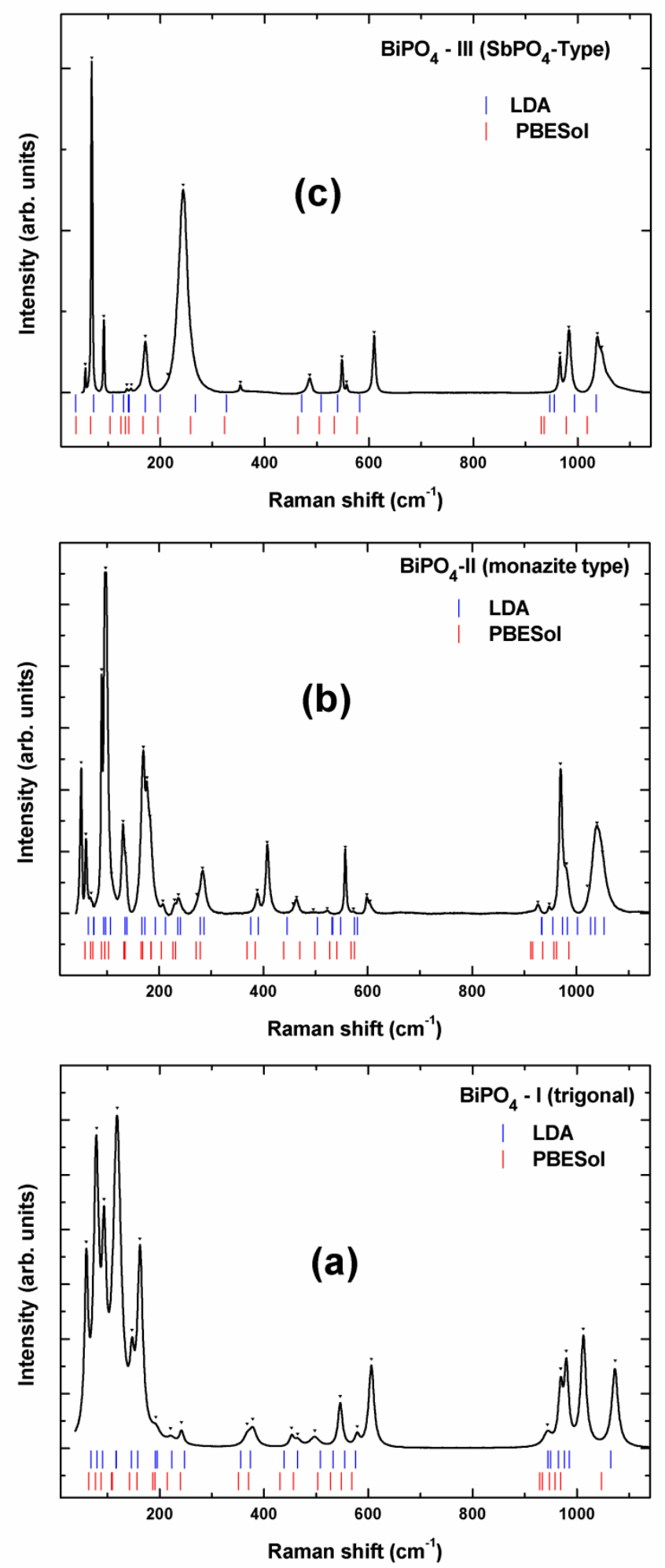

Fig. 10: Typical Raman spectra of $\mathrm{BiPO}_{4}$ phases. (a). Trigonal (I), (b). Monazite-type (II) and (c). SbPO 4 -type (III). Vertical ticks indicate the calculated frequencies. 
( $v_{4}$ bands) $\mathrm{cm}^{-1}$. Additional new bands at 669, $632 \mathrm{~cm}^{-1}$ might be arising from the $v_{3}$ and $v_{1}$ modes of $\mathrm{PO}_{4}$ units.

As regards Raman scattering measurements of $\mathrm{BiPO}_{4}$ polymorphs presented in Fig. 10, it must be emphasized here that Raman data of $\mathrm{BiPO}_{4}$ already reported in the literature are obscure in its interpretation, though they have been often used to characterize the $\mathrm{BiPO}_{4}$ phases. ${ }^{16-19,}{ }^{32}$, 58, 59 In most cases, the high-frequency Raman modes of free $\mathrm{PO}_{4}$ units were used to characterize the different phases, but a complete mode analysis has not been undertaken to our knowledge. Here we present a detailed symmetry analysis and computational study which has allowed us to identify almost all the Raman modes of the $\mathrm{BiPO}_{4}$ phases (see Table 5(a-c)). In the present study, about 20 modes could be clearly identified in the Raman spectra of $\mathrm{BiPO}_{4}-\mathrm{I}$. As expected, internal vibrational modes of $\mathrm{PO}_{4}$ units appear at high frequencies, while those due to the internal modes of the $\mathrm{BiO}_{8}$ units are observed at low frequency. The intense $\mathrm{A}_{1}\left(118.7 \mathrm{~cm}^{-1}\right.$, strong and sharp) and $\mathrm{E}$ (241.7 $\mathrm{cm}^{-1}$, strong and broad) modes are due to the internal modes of $\mathrm{BiO}_{8}$ polyhedra in $\mathrm{BiPO}_{4}$-I. ${ }^{60}$ The five modes observed at 944, 969, 980, 1012 and $1073 \mathrm{~cm}^{-1}$ are assigned to the symmetric and asymmetric stretching frequencies of $\mathrm{P}-\mathrm{O}$ bonds of $\mathrm{PO}_{4}$ units of the trigonal phase. Similar comparison with the earlier reported Raman modes of $\mathrm{PO}_{4}$ groups, the bands observed at 498, 546, 579 and $606 \mathrm{~cm}^{-1}$ can be assigned to the bending (v4) modes and bands at $379,453,465 \mathrm{~cm}^{-1}$ can be assigned to the (v2) bending modes of $\mathrm{PO}_{4}$ units. The stretching modes of bonds in $\mathrm{PO}_{4}$ units in monazite (II) phase are also quite similar to those observed for trigonal (I) phase except that for some splitting. This can be attributed to highly dissimilar bond lengths in phases-I and II as described in the explanation of IR results. Similar split bands are also observed at lower frequencies due to the internal modes of the $\mathrm{BiO}_{\mathrm{n}}$ units of phase II and III. In particular the phase II and III display an additional band around $400 \mathrm{~cm}^{-1}$ compared to the trigonal (I) phase. Earlier, such band was observed by Geng et al. ${ }^{58}$ and Frost et al. ${ }^{60}$ and had been assigned to the $\mathrm{Bi}-\mathrm{O}$ stretching frequency of $\mathrm{BiO}_{\mathrm{n}}$ polyhedra. This intensity and shift in the frequencies can be assigned to the average $\mathrm{Bi}-\mathrm{O}$ bond length and compactness of the polyhedra as the structure changes from trigonal to monazite-type or $\mathrm{SbPO}_{4}$-type structures. 


\section{Conclusions}

In summary, the detailed analyses of the powder X-ray and neutron diffraction data as well as IR and Raman studies on three polymorphs of $\mathrm{BiPO}_{4}$ revealed accurate structural parameters and local distortions parameters around $\mathrm{Bi}^{3+}$ and $\mathrm{P}^{5+}$. Further the structural parameters and vibrational frequencies were supported by $a b$ initio calculations based on DFT. The irreversible trigonal-monazite- $\mathrm{SbPO}_{4}$-type structural transitions are explained from the molar volume and total energy of the three phases. Highly distorted structural arrangement around $\mathrm{Bi}^{3+}$ and $\mathrm{P}^{5+}$ has a very limited stability and is only stabilized by water molecules in the trigonal phase. It is revealed that structural transition from trigonal to monazite or $\mathrm{SbPO}_{4}$ type structures is accompanied by a significant reduction in volume. DFT calculations revealed that the monazite and $\mathrm{SbPO}_{4}$-type $\mathrm{BiPO}_{4}$ have almost similar total energy and hence a competing stability. Further it is observed that monazite type phase shows larger lattice thermal expansion compared to the $\mathrm{SbPO}_{4}$-type phase and thus it is truly a metastable phase.

\section{Acknowledgements}

This study was supported by the Spanish government MEC under Grants No: MAT2010-21270C04-01/04, by MALTA Consolider Ingenio 2010 project (CSD2007-00045), and by the Vicerrectorado de Investigación y Desarrollo of the Universidad Politécnica de Valencia (UPV2011-0914 PAID-05-11 and UPV2011-0966 PAID-06-11). S.N.A. acknowledges the support provided by Universitat de Valencia during his visit to it. A.M. and P.R-H. acknowledge computing time provided by Red Española de Supercomputación (RES) and MALTA-Cluster.

†Electronic Supplementary Information (ESI) available: [(ESI-1). Further details of crystal structural data of the BiPO4-I, II and III can be obtained from the Fachinformationszentrum Karlsruhe, Abt. PROKA, 76344 Eggenstein-Leo-poldshafen, Germany (fax +49-7247-808-666; E-mail: crysdata@fiz-karlsruhe.de) on quoting the depository numbers CSD 426230 to 426255 (26 structures)]. See DOI: 10.1039/b000000x 


\section{References}

1. L. A. Boatner and B. C. Sales, in "Radioactive Waste Forms for the Future” 1988, p. 495. Eds. W. Lutze and R. C. Ewing, North-Holland, Amsterdam.

2. L. Bois, M. J. Guitter, F. Crrot, P. Trocellier, and M. Gautier-Soyer, J. Nucl. Mater. 2001, 297, 129.

3. N. Dacheux, A. C. Thomas, V. Brandel, and M. Genet, J. Nucl. Mater. 1998, 257, 108.

4. N. Clavier, N. Dacheux, and N. Podor, Inorg. Chem. 2006, 45, 220.

5. $\quad$ N. Clavier, N. Dacheux, C. Wallez, and M. Quarton, J. Nucl. Mater. 2006, 352, 209.

6. $\quad$ K. R. ELaud and F. A. Hummel, J. Am. Ceram. Soc. 1971, 54, 296.

7. O. Muller and R. Roy, The Major Ternary Structural Families (Springer, Berlin, 1974).

8. M. J. Kohn, J. Rakovan, and J. M. Hughes, Phosphates: Geochemical, Geobiological, and Materials Importance, vol. 48, p. 87-123. (Ed), Reviews in Mineralogy and Geochemistry, Mineralogical Society of America and the Geochemical Society, Chantilly, Virginia.

9. D. Errandonea and F. J. Manjon, Prog. Mater. Sci. 2008, 53, 711.

10. O. Fukunaga and S. Yamaoka, Phys. Chem. Miner 1979, 5, 167.

11. E. H. Oelkers and J. -M. Montel, Elements 2008, 4, 113.

12. G. J. McCarthy, W. B. White, R. Roy, B. E. Scheetz, S. Komarneni, S. K. Smith, and D. M. Roy, Nature 1978, 273, 316.

13. G. J. McCarthy, W. B. White, and D. E. Pfoertsch, Mater. Res. Bull. 1978, 13, 1239.

14. I. -S. Cho, G. K. Choi, J. -S. An, J. -R. Kim, and K. S. Hong, Mater. Res. Bull. 2009, 44, 173.

15. N. Kitamura, K. Amezawa, Y. Tomii, and N. Yamamotoa, Sol. State Ionics 2003, 161,162.

16. C. Pan, D. Li, X. Ma, Y. Chen, and Y. Zhu, Catal. Sci. Tech. 2011, 1, 1399.

17. C. Pan, J. Xu, Y. Chen, and Y. Zhu, Appl. Catal. B: Environmental 2012, 115, 314.

18. M. Zhao, G. Li, J. Zheng, L. Li, H. Wang, and L. Yang, Cryst. Engg 2011, 13, 6251.

19. P. Arunkumar, C. Jayajothi, D. Jeyakumar, and N. Lakshminarasimhan, RSC Adv 2012, 2, 1477.

20. V. G. Alekseev, I. P. Gorelov, and M. V. Kornilov, J. Anal. Chem. 20005, 5, 1055. 
21. I. -S. Cho, J. R. Kim, D. W. Kim, and K. S. Hong, J. Electroceram 2006, 16, 379.

22. Z. Holgye and R. Poliak, J. Radioanal. Nucl. Chem. 1991, 153, 267.

23. Z. Holgye, J. Radioanal. Nucl. Chem 1998, 12, 227.

24. C. Pan and Y. Zhu, Environ. Sci. Technol 2010, 44, 5570.

25. C. Pan and Y. Zhu, Y. J. Mater. Chem. 2011, 21, 4235.

26. R. C. L. Mooney-Slater, Z. Krist. 1972, 117, 371.

27. G. Chiari and G. Ferraris, Acta Cryst. B 1982, 38, 2331.

28. M. Roming and C. Feldmann, J. Mater. Sci 2009, 44, 1412.

29. Y. -F. Lin, H. -W. Chang, S. -Y. Lu, and C. W. Liu, J. Phys. Chem. C 2007, 111, 18538.

30. I.-S. Cho, J.-R. Kim, D.-W. Kim, and K. S. Hong, J. Electroceram 2006, 16, 379.

31. R. Masse and A. C. Durif, C. R. acad of Sci. Paris, 1985, 300, 349.

32. B. Romero, S. Bruque, M. A. G. Aranda, and J. E. Iglesias, Inorg. Chem. 1994, 33, 1869.

33. B. S. Naidu, B. Vishwanadh, V. Sudarsan, and R. K. Vatsa, Dalton Trans 2012, 41, 3194.

34. M. Zhao, L. Li, J. Zheng, L. Yang, and G. Li, Inorg. Chem. 2013, 52, 807.

35. A. C. Larson and R. B. van Dreele, GSAS: General Structure Analysis System. Los Alamos National Laboratory, Report LA-UR 86-748, 2000.

36. J. Rodriguez-Carvajal, Fullprof 2000: A Program for Rietveld, Profile Matching and Integrated Intensity Refinements for X-ray and Neutron Data. Version 1.6, Laboratoire Leon Brillouin, Gif sur Yvette, France, 2000.

37. G. Kresse and J. Furthmuller, Phys. Rev. B 1996, 54, 11169.

38. G. Kresse and D. Joubert, Phys. Rev. B 1999, 59, 1758.

39. P. E. Blochl, Phys.Rev. B 1994, 50, 17953.

40. J. P. Perdew and A. Zunger, Phys. Rev. B, 1981, 23, 5048.

41. J. P. Perdew, K. Burke, and M. Ernzerhof, Phys. Rev. Lett. 1996, 77, 3865.

42. A. Mujica, A. Rubio, A. Muñoz, and R. J. Needs, Rev. Mod. Phys. 2003, 75, 863.

43. K. Parlinski, computer code PHONON [http://wolf.ifj.edu.pl/phonon/].

44. F. Xue, H. Li, Y. Zhu, S. Xiong, X. Zhang, T. Wang, X. Liang, and Y. Qian, J. Solid State Chem. 2009, 182, 1396.

45. $\quad$ S. J. Patwe, S. N. Achary, and A. K. Tyagi, J. Mater. Res., 2009, 24, 3551.

46. S. N. Achary, S. J. Patwe, M. D. Mathews, and A. K Tyagi, J. Phys. Chem. Solids, 2006, 67, 774. 
47. S. J. Patwe, S. N. Achary, and A. K. Tyagi, Am. Mineralogist, 2009, 94, 98.

48. R. Lacomba-Perales, D. Errandonea, Y. Meng, and M. Bettinelli, Phys. Rev. B 2010, 81, 064113.

49. V. Panchal, S. López-Moreno, D. Santamaŕia-Pérez, D. Errandonea, D. J. Manóon, P. Rodŕrguez-Hernandez, A. Mũnoz, S. N. Achary, and A. K. Tyagi, Phys. Rev. B 2011, 84, 024111.

50. D. Errandonea, R. S. Kumar, S. N. Achary, and A. K. Tyagi, Phys. Rev. B 2011, 84, 224121.

51. L. Gracia, A. Beltran, D. Errandonea, and J. Andres, Inorg. Chem. 2012, 51, 1751.

52. T. B. Zunic and L. Vickovic, J. Appl. Cryst. 1996, 29, 305.

53. $\quad$ E. Makovicky and T. B. Zunic, Acta Cryst. B 1998, 54, 766.

54. M. E. Poloznikova and V. V. Fomichev, Usp. Khim., 1994, 63, 419.

55. L. Baia, R. Stefan, W. Kiefer, J. Popp, and S. Simon, J. Non-Cryst. Solids, 2002, 303, 379.

56. S. D. Ross, Inorganic Infrared and Raman Spectra (McGraw-Hill, London, 1972).

57. K. Nakamoto, Infrared and Raman spectra of Inorganic and Coordination Compounds (Wiley, New York, 1986).

58. J. Geng, W. H. Hou, Y. N. Lv, J. J. Zhu, and H. Y. Chen, Inorg. Chem. 2005, 44, 8503.

59. G. M. Begun, G. W. Beall, L. A. Boatner, and W. J. Gregor, J. Raman Spect. 1981, 11, 273.

60. R. L. Frost, M. L. Weier, K. L. Erickson, O. Carmody, and S. J. Mills, J. Raman Spect. 2004, 35, 1047. 


\section{Figure Captions}

Fig. 1: Powder XRD patters of different phases of $\mathrm{BiPO}_{4}$ at ambient temperature $(\lambda=1.5417 \AA)$.

Fig. 2: Rietveld refinement plots of powder neutron diffraction data of $\mathrm{BiPO}_{4}$ phases, (a).

Trigonal (I), (b). Monazite-type (II) and (c). SbPO 4 -type (III). $(\lambda=1.249 \AA$ ).

Fig. 3: Crystal structure of $\mathrm{BiPO}_{4}$ phases, (a). Trigonal (I), (b). Monazite-type (II) and (c). SbPO 4 -type (III). ( $\mathrm{BiO}_{8}$ (in a and c), $\mathrm{BiO}_{9}$ (in b) and $\mathrm{PO}_{4}$ units are shown). (isolated spheres are O3(of water molecules in $\mathrm{BiPO}_{4}-\mathrm{I}$ ).

Fig. 4: Typical coordination around $\mathrm{Bi}^{3+}$ in $\mathrm{BiPO}_{4}$ phases, (a). Trigonal (I), (b). Monazite type (II) and (c). $\mathrm{SbPO}_{4}$ type (III)

Fig. 5: Powder XRD patterns of $\mathrm{BiPO}_{4}$ phases at representative temperatures. (a). Trigonal (I), (b). Monazite-type (II) and (c). $\mathrm{SbPO}_{4}$-type (III) ( $\uparrow$ indicate platinum sample holder base peaks)

Fig. 6: Variation of unit cell parameters of $\mathrm{BiPO}_{4}$ with temperature. (a). Trigonal (I), (b). Monazite-type (II) and (c). SbPO 4 -type (III).

Fig. 7: Variation of molar volume of $\mathrm{BiPO}_{4}$ phases with temperature.

Fig. 8: Total energy as calculated by LDA and PBESol approach.

Fig. 9: Typical FTIR spectra of $\mathrm{BiPO}_{4}$ phases. (a). Trigonal (I), (b). Monazite-type (II) and (c). $\mathrm{SbPO}_{4}$-type (III). Vertical ticks indicate the calculated frequencies.

Fig. 10: Typical Raman spectra of $\mathrm{BiPO}_{4}$ phases. (a). Trigonal (I), (b). Monazite-type (II) and (c). $\mathrm{SbPO}_{4}$-type (III). Vertical ticks indicate the calculated frequencies. 


\section{Tables}

Table 1: Refined unit cell parameters for different polymorphs of $\mathrm{BiPO}_{4}$ at ambient temperature (300K). (Calculated unit cell parameters are also included for comparison)

Table-2: Refined and calculated structural parameters of $\mathrm{BiPO}_{4}$ at $300 \mathrm{~K}((\mathrm{a})$. Trigonal (I), (b). Monazite-type (II) and (c). SbPO 4 -type (III)).

Table 3: The typical bond lengths and polyhedral parameters in different phases of $\mathrm{BiPO}_{4}$.

Table 4: Calculated and observed Infrared modes of different $\mathrm{BiPO}_{4}$ phases. ((a). Trigonal (I), (b). Monazite-type (II) and (c). SbPO4-type (III).)

Table 5: Calculated and observed Raman modes of $\mathrm{BiPO}_{4}$ phases. ((a). Trigonal (I), (b). Monazite-type (II) and (c). SbPO 4 -type (III)). 
Table 1: Refined unit cell parameters for different polymorphs of $\mathrm{BiPO}_{4}$ at ambient temperature (300K). (Calculated unit cell parameters are also included for comparison)

\begin{tabular}{|c|c|c|c|c|c|c|c|c|c|c|c|c|}
\hline & \multicolumn{4}{|c|}{$\mathrm{BIPO}_{4}-\mathrm{I} \mathrm{BiPO}_{4} 0.67 \mathrm{H}_{2} \mathrm{O}$ (trigonal) } & \multicolumn{4}{|c|}{$\mathrm{BIPO}_{4}$-II BiPO 4 (Monazite-type) } & \multicolumn{4}{|c|}{$\mathrm{BIPO}_{4}$-III BiPO ${ }_{4}\left(\mathrm{SbPO}_{4}\right.$-type $)$} \\
\hline & \multicolumn{4}{|c|}{ Space group: $\mathrm{P} 3121$ (No. 152) } & \multicolumn{4}{|c|}{ Space group: $\mathrm{P} 2_{1} / \mathrm{n}$ (No. 14) } & \multicolumn{4}{|c|}{ Space group: $\mathrm{P} 2_{1} / \mathrm{m}$ (No. 11$)$} \\
\hline & ND & XRD & LDA & PBESol & ND & XRD & LDA & PBESol & ND & XRD & LDA & PBESol \\
\hline $\mathrm{a}(\AA)$ & $6.9793(5)$ & $6.9813(2)$ & 6.9151 & 6.9882 & $6.7561(4)$ & $6.7552(1)$ & 6.6658 & 6.7549 & $4.8807(2)$ & $4.8804(1)$ & 4.7375 & 4.7809 \\
\hline $\mathrm{b}(\AA)$ & & & & & 6.9408(4), & $6.9417(2)$ & 6.8869 & 6.9551 & 7.0674(3) & $7.0684(2)$ & 7.0726 & 7.1590 \\
\hline c $(\AA)$ & $6.4743(7)$ & $6.4751(2)$ & 6.3798 & 6.4314 & $6.4764(3)$ & $6.4772(2)$ & 6.4148 & 6.4700 & $4.7023(2)$ & 4.7033(1) & 4.6387 & 4.7011 \\
\hline$\beta\left(^{\circ}\right)$ & & & & & 103.695(5) & 103.691(2) & 103.79 & 103.95 & $96.303(4)$ & $96.285(3)$ & 95.91 & 96.07 \\
\hline $\mathrm{V}\left(\AA^{3}\right)$ & 273.11(3) & 273.31(1) & 264.20 & 272.00 & $295.06(2$ & $295.10(1)$ & 286.00 & 295.00 & $161.22(1)$ & $161.27(1)$ & 154.6 & 160.00 \\
\hline $\mathrm{Z}$ & 3 & & & & 4 & & & & 4 & & & \\
\hline $\mathrm{V} / \mathrm{Z}\left(\AA^{3}\right)$ & 91.04 & & & & 73.77 & & & & 80.61 & & & \\
\hline $\mathrm{R}_{\mathrm{p}}, \mathrm{R}_{\mathrm{wp}}$ & $\begin{array}{l}0.0164 \\
0.0216\end{array}$ & & & & $\begin{array}{l}0.0276 \\
0.0351\end{array}$ & & & & $\begin{array}{l}0.0385 \\
0.0517\end{array}$ & & & \\
\hline$\chi^{2}$ & 0.096 & & & & 1.073 & & & & 2.027 & & & \\
\hline $\mathrm{R}_{\mathrm{F}}^{2}$ & 0.0813 & & & & 0.0578 & & & & 0.0428 & & & \\
\hline
\end{tabular}


Table-2: Refined and calculated structural parameters of $\mathrm{BiPO}_{4}$ at $300 \mathrm{~K}((\mathrm{a})$. Trigonal (I), (b). Monazite-type (II) and (c). SbPO4-type (III)).

(a). $\mathrm{BiPO}_{4}$-I. (Trigonal)

\begin{tabular}{lllllll}
\hline & wyc & $\boldsymbol{x}$ & $\boldsymbol{y}$ & $\boldsymbol{z}$ & $\boldsymbol{o c c}$ & Uiso $\AA^{2}$ \\
\hline $\mathrm{Bi}$ & $3 b$ & $\mathbf{0 . 4 7 0 3 ( 9 )}$ & $\mathbf{0}$ & $\mathbf{5 / 8}$ & $\mathbf{1}$ & $\mathbf{0 . 0 0 7 7 ( 1 3 )}$ \\
& & 0.47727 & 0.00000 & 0.83333 & & \\
$\mathrm{P}$ & $3 a$ & 0.48136 & 0.00000 & 0.83333 & & $\mathbf{0 . 0 0 9 3 ( 1 7 )}$ \\
& & $\mathbf{0 . 4 4 0 1 ( 1 5 )}$ & $\mathbf{0}$ & $\mathbf{1} / \mathbf{3}$ & $\mathbf{1}$ & \\
$\mathrm{O} 1$ & & 0.44011 & 0.00000 & 0.33333 & & \\
& $6 c$ & 0.44194 & 0.00000 & 0.33333 & & $\mathbf{0 . 0 2 3 1 ( 1 8 )}$ \\
& & $\mathbf{0 . 3 9 3 9 ( 1 2 )}$ & $\mathbf{0 . 1 5 6 9 ( 1 5 )}$ & $\mathbf{0 . 4 5 9 2 ( 1 0 )}$ & $\mathbf{1}$ & \\
$\mathrm{O} 2$ & & 0.60625 & 0.15651 & 0.19788 & & $\mathbf{0 . 0 1 5 2 ( 1 7 )}$ \\
& $6 c$ & 0.60569 & 0.15485 & 0.19843 & & \\
$\mathrm{O} 3(\mathrm{w})$ & & $\mathbf{0 . 6 2 7 0 ( 1 2 )}$ & $\mathbf{0 . 1 3 2 4 ( 1 2 )}$ & $\mathbf{0 . 1 6 5 2 ( 1 1 )}$ & $\mathbf{1}$ & \\
& & 0.49845 & 0.12767 & 0.49385 & & $\mathbf{0 . 0 1 1 ( 5 )}$ \\
& & 0.49651 & 0.12678 & 0.49308 & & \\
\hline
\end{tabular}

$\mathbf{1}^{\text {st }}$ row : Refined parameters from powder neuron diffraction data

$2^{\text {nd }}$ row : $\operatorname{LDA}\left(a=b=6.91509 \AA, c=6.37979 \AA, \gamma=120^{\circ}\right)$

$3^{\text {rd }}$ row : PBESol. $\left(\mathrm{a}=\mathrm{b}=6.98821 \AA, \mathrm{c}=6.43141 \AA, \gamma=120^{\circ}\right)$

(Calculated Structures at 0 pressure and $0 \mathrm{~K}$ )

* Note that in the trigonal structure we neglected the presence of the $\mathrm{H}_{2} \mathrm{O}$ molecule in calculations. 
(b). $\mathrm{BiPO}_{4}$-II (Monazite-type)

\begin{tabular}{|c|c|c|c|c|c|c|}
\hline & wyc & $x$ & $y$ & $Z$ & OCC & Uiso $\left(\AA^{2}\right)$ \\
\hline \multirow[t]{3}{*}{$\mathrm{Bi}$} & $4 e$ & $0.2871(5)$ & $0.1447(5)$ & $0.0859(5)$ & 1 & $0.0092(6)$ \\
\hline & & 0.2824 & 0.1462 & 0.0906 & & \\
\hline & & 0.28394 & 0.14513 & 0.08817 & & \\
\hline \multirow[t]{3}{*}{$\mathrm{P}$} & $4 e$ & $0.2966(8)$ & $0.1615(5)$ & $0.6163(7)$ & 1 & $0.0073(9)$ \\
\hline & & 0.2995 & 0.1622 & 0.6141 & & \\
\hline & & 0.29933 & 0.16215 & 0.61291 & & \\
\hline \multirow[t]{3}{*}{ O1 } & $4 e$ & $0.2617(6)$ & $0.0003(6)$ & $0.4459(8)$ & 1 & $0.0110(9)$ \\
\hline & & 0.2594 & 0.0041 & 0.4411 & & \\
\hline & & 0.25990 & 0.00537 & 0.44010 & & \\
\hline \multirow[t]{3}{*}{$\mathrm{O} 2$} & $4 e$ & $0.3771(6)$ & $0.3410(7)$ & $0.5143(6)$ & 1 & $0.0067(7)$ \\
\hline & & 0.3799 & 0.3422 & 0.5148 & & \\
\hline & & 0.37939 & 0.34210 & 0.51574 & & \\
\hline \multirow[t]{3}{*}{ O3 } & $4 e$ & $0.4618(6)$ & $0.1029(7)$ & $0.8136(7)$ & 1 & $0.0025(8)$ \\
\hline & & 0.4664 & 0.1027 & 0.8189 & & \\
\hline & & 0.46480 & 0.10182 & 0.81782 & & \\
\hline \multirow[t]{3}{*}{$\mathrm{O} 4$} & $4 e$ & $0.1161(7)$ & $0.2011(7)$ & $0.7097(7)$ & 1 & $0.0067(9)$ \\
\hline & & 0.1144 & 0.2059 & 0.7113 & & \\
\hline & & 0.11546 & 0.20505 & 0.70932 & & \\
\hline
\end{tabular}

$\mathbf{1}^{\text {st }}$ row : Refined parameters from powder neuron diffraction data

$2^{\text {nd }}$ row : $L D A(\mathrm{P} 21 / \mathrm{n}$, setting 7$\left.) \mathrm{a}=6.6658 \AA, \mathrm{b}=6.8869 \AA, \mathrm{c}=6.4148 \AA, \beta=103.79^{\circ}\right)$

$3^{\text {rd }}$ row : PBESol. $(P 21 / n$, setting 7$) a=6.7549 \AA, b=6.9551 \AA, c=6.4700 \AA, \beta=103.95^{\circ}$ )

(Calculated structures at 0 pressure and $0 \mathrm{~K}$ ) 
(c). $\mathrm{BiPO}_{4}$-III (SbPO 4 -type)

\begin{tabular}{|c|c|c|c|c|c|c|}
\hline & wyc & $x$ & $y$ & $\mathbf{Z}$ & occ & Uiso $\left(\AA^{2) \#}\right.$ \\
\hline \multirow[t]{3}{*}{$\mathrm{Bi}$} & $2 \mathrm{e}$ & $0.1424(4)$ & 0.2500 & $0.1671(5)$ & 1 & 0.00875 \\
\hline & & 0.12747 & 0.25000 & 0.15446 & & \\
\hline & & 0.13160 & 0.25000 & 0.14926 & & \\
\hline \multirow[t]{3}{*}{$\mathrm{P}$} & $2 \mathrm{e}$ & $0.3733(7)$ & 0.7500 & $0.3027(7)$ & 1 & 0.00403 \\
\hline & & 0.63319 & 0.25000 & 0.67447 & & \\
\hline & & 0.63746 & 0.25000 & 0.67464 & & \\
\hline \multirow[t]{3}{*}{$\mathrm{O} 1$} & $2 e$ & $0.6761(5)$ & 0.7500 & $0.2291(7)$ & 1 & 0.01239 \\
\hline & & 0.31724 & 0.25000 & 0.72805 & & \\
\hline & & 0.32318 & 0.25000 & 0.72855 & & \\
\hline \multirow[t]{3}{*}{$\mathrm{O} 2$} & $2 \mathrm{e}$ & $0.6013(6)$ & 0.2500 & $0.3655(6)$ & 1 & 0.01373 \\
\hline & & 0.63648 & 0.25000 & 0.34423 & & \\
\hline & & 0.64067 & 0.25000 & 0.34750 & & \\
\hline \multirow[t]{3}{*}{$\mathrm{O} 3$} & $4 \mathrm{f}$ & $0.2102(4)$ & $0.5802(4)$ & $0.1750(5)$ & 1 & 0.01231 \\
\hline & & 0.20392 & 0.57963 & 0.18037 & & \\
\hline & & 0.20006 & 0.58078 & 0.18053 & & \\
\hline
\end{tabular}

$1^{\text {st }}$ row : Refined parameters from powder neuron diffraction data

$2^{\text {nd }}$ row : $L D A\left(\mathrm{P} 2_{1} / \mathrm{m}, \mathrm{a}=4.73755 \AA, \mathrm{b}=7.07260 \AA, \mathrm{c}=4.63868 \AA, \beta=95.91397^{\circ}\right)$

$3^{\text {rd }}$ row : PBASol. $\left(\mathrm{P} 2{ }_{1} / \mathrm{m}, \mathrm{a}=4.78092 \AA, \mathrm{b}=7.15898 \AA, \mathrm{c}=4.70108 \AA, \beta=96.06833^{\circ}\right)$

(Calculated Structures at 0 pressure and $0 \mathrm{~K}$ )

\# Anisotropic thermal parameters for $\mathrm{BiPO}_{4}\left(\mathrm{SbPO}_{4}\right.$-type $)$

\begin{tabular}{lllllll}
\hline & $\boldsymbol{U}_{1 \mathbf{1 1}}\left(\AA^{2}\right)$ & $\boldsymbol{U}_{\mathbf{1 2}}\left(\AA^{2}\right)$ & $\boldsymbol{U}_{\mathbf{1 3}}\left(\AA^{2}\right)$ & $\boldsymbol{U}_{\mathbf{2 2}}\left(\AA^{2}\right)$ & $\boldsymbol{U}_{23}\left(\AA^{2}\right)$ & $\boldsymbol{U}_{33}\left(\AA^{2}\right)$ \\
\hline $\mathrm{Bi}$ & $0.0129(13)$ & 0.0 & $0.0003(9)$ & $0.0022(13)$ & 0.0 & $0.0110(11)$ \\
$\mathrm{P}$ & $0.0051(16)$ & 0.0 & $-0.0018(13)$ & $0.0064(15)$ & 0.0 & $0.0001(17)$ \\
$\mathrm{O} 1$ & $0.0014(14)$ & 0.0 & $-0.0011(13)$ & $0.0262(17)$ & 0.0 & $0.0092(19)$ \\
$\mathrm{O} 2$ & $0.0059(16)$ & 0.0 & $0.0042(15)$ & $0.0228(19)$ & 0.0 & $0.0132(19)$ \\
$\mathrm{O} 3$ & $0.0134(12)$ & $0.0012(9)$ & $-0.0037(9)$ & $0.0101(12)$ & $-0.0050(10)$ & $0.0124(11)$ \\
\hline
\end{tabular}


Table 3: The typical bond lengths and polyhedral parameters in different phases of $\mathrm{BiPO}_{4}$.

\begin{tabular}{|c|c|c|c|c|c|}
\hline \multicolumn{2}{|c|}{$\mathrm{BIPO}_{4}-\mathrm{I}$ (Trigonal) } & \multicolumn{2}{|c|}{$\mathrm{BIPO}_{4}$-II (Monazite-type) } & \multicolumn{2}{|c|}{$\mathrm{BIPO}_{4}$-III $\left(\mathrm{SbPO}_{4}\right.$-type $)$} \\
\hline $\mathrm{Bi}-\mathrm{O} 1$ & $2.817(8)(\AA)$ & $\mathrm{Bi}-\mathrm{O} 1$ & $2.580(6)(\AA)$ & $\mathrm{Bi}-\mathrm{O} 1$ & $2.149(4)(\AA)$ \\
\hline $\mathrm{Bi}-\mathrm{O} 1$ & $2.296(8)$ & $\mathrm{Bi}-\mathrm{O} 1$ & $2.492(5)$ & $\mathrm{Bi}-\mathrm{O} 1$ & $2.880(4)$ \\
\hline $\mathrm{Bi}-\mathrm{O} 1$ & $2.817(7)$ & $\mathrm{Bi}-\mathrm{O} 2$ & $3.020(5)$ & $\mathrm{Bi}-\mathrm{O} 2$ & $2.896(4)$ \\
\hline $\mathrm{Bi}-\mathrm{O} 1$ & $2.296(12)$ & $\mathrm{Bi}-\mathrm{O} 2$ & $2.399(6)$ & $\mathrm{Bi}-\mathrm{O} 2$ & $2.330(3)$ \\
\hline $\mathrm{Bi}-\mathrm{O} 2$ & $2.378(7)$ & $\mathrm{Bi}-\mathrm{O} 2$ & $2.700(5)$ & $\mathrm{Bi}-\mathrm{O} 3$ & $2.357(3)$ \\
\hline $\mathrm{Bi} 1-\mathrm{O} 2$ & $2.490(9)$ & $\mathrm{Bi}-\mathrm{O} 3$ & $2.361(6)$ & $\mathrm{Bi}-\mathrm{O} 3$ & $2.526(3)$ \\
\hline $\mathrm{Bi}-\mathrm{O} 2$ & $2.490(7)$ & $\mathrm{Bi}-\mathrm{O} 3$ & $2.394(6)$ & $\mathrm{Bi}-\mathrm{O} 3$ & $2.526(3)$ \\
\hline \multirow[t]{2}{*}{$\mathrm{Bi}-\mathrm{O} 2$} & $2.378(8)$ & $\mathrm{Bi}-\mathrm{O} 4$ & $2.469(5))$ & $\mathrm{Bi}-\mathrm{O} 3$ & $2.357(3)$ \\
\hline & & $\mathrm{Bi}-\mathrm{O} 4$ & $2.426(6)$ & & \\
\hline CN: & $6+2$ & $\mathrm{CN}$ & $8+1$ & $\mathrm{CN}$ & 8 \\
\hline$<\mathrm{Bi}-\mathrm{O}>$ & 2.495(3) ( $)$ & $<\mathrm{Bi}-\mathrm{O}>_{9}$ & $2.538(2)(\AA)$ & $<\mathrm{Bi}-\mathrm{O}>$ & $2.503(1)(\AA)$ \\
\hline \multirow[t]{3}{*}{ Distt. } & $62.976 \times 10^{-4}$ & Distt. & $60.598 \times 10^{-4}$ & Distt. & $98.915 \times 10^{-4}$ \\
\hline & & $<\mathrm{Bi}-\mathrm{O}>_{8}$ & $2.478(2)(\AA)$ & & \\
\hline & & Distt. & $18.238 \times 10^{-4}$ & & \\
\hline $\mathrm{V}_{\mathrm{BiO} 8}\left(\AA^{3}\right)$ & 29.06 & $\mathrm{~V}_{\mathrm{BiO} 8}\left(\AA^{3}\right)$ & 26.26 & $\mathrm{~V}_{\mathrm{BiO} 8}\left(\AA^{3}\right)$ & 27.28 \\
\hline Distt. & 0.1491 & Distt. & 0.0684 & Distt. & 0.0819 \\
\hline Sphericity & 0.844 & Sphericity & 0.941 & Sphericity & 0.910 \\
\hline \multirow[t]{5}{*}{ Eccentricity } & 0.346 & Eccentricity & 0.196 & Eccentricity & 0.449 \\
\hline & & $\mathrm{V}_{\mathrm{BiO}}\left(\AA^{3}\right)$ & 31.95 & & \\
\hline & & Distt. & 0.0557 & & \\
\hline & & Sphericity & 0.853 & & \\
\hline & & Eccentricity & 0.308 & & \\
\hline $\mathrm{P}-\mathrm{O} 1$ & $1.523(12)(\AA)$ & $\mathrm{P}-\mathrm{O} 1$ & $1.550(7)(\AA)$ & P - O1 & $1.554(4)(\AA)$ \\
\hline P - O1 & $1.523(9)$ & $\mathrm{P}-\mathrm{O} 2$ & $1.567(7)$ & $\mathrm{P}-\mathrm{O} 2$ & $1.552(4)$ \\
\hline $\mathrm{P}-\mathrm{O} 2$ & 1.592(9) & $\mathrm{P}-\mathrm{O} 3$ & $1.539(6)$ & $\mathrm{P}-\mathrm{O} 3$ & $1.526(3)$ \\
\hline $\mathrm{P}-\mathrm{O} 2$ & $1.592(10)$ & $\mathrm{P}-\mathrm{O} 4$ & $1.509(8)$ & $\mathrm{P}-\mathrm{O} 3$ & $1.526(3)$ \\
\hline$<\mathrm{P}-\mathrm{O}>_{4}$ & $1.558(5)(\AA)$ & $<\mathrm{P}-\mathrm{O}>_{4}$ & $1.541(4)(\AA)$ & $<\mathrm{P}-\mathrm{O}>$ & $1.540(2)(\AA)$ \\
\hline Distt. & $4.895 \times 10^{-4}$ & Distt. & $1.839 \times 10^{-4}$ & Distt. & $0.757 \times 10^{-4}$ \\
\hline $\mathrm{V}_{\mathrm{PO} 4}\left(\AA^{3}\right)$ & 1.923 & $\mathrm{~V}_{\mathrm{PO} 4}\left(\AA^{3}\right)$ & 1.862 & $\mathrm{~V}_{\mathrm{PO} 4}\left(\AA^{3}\right)$ & 1.861 \\
\hline Distt. & 0.0128 & Distt. & 0.0094 & Distt. & 0.0070 \\
\hline Sphericity & 1 & Sphericity & 1 & Sphericity & 1 \\
\hline Eccentricity & 0.042 & Eccentricity & 0.066 & Eccentricity & 0.116 \\
\hline
\end{tabular}


Table 4: Calculated and observed Infrared modes of different $\mathrm{BiPO}_{4}$ phases. ((a). Trigonal (I), (b). Monazite-type (II) and (c). SbPO4-type (III).)

(a). Trigonal $\left(\mathrm{BiPO}_{4}-\mathrm{I}\right)$

\begin{tabular}{|c|c|c|c|c|c|}
\hline Mode & $\operatorname{LDA} \omega\left(\mathrm{cm}^{-1}\right)$ & Mode & PBESol $\omega\left(\mathrm{cm}^{-1}\right)$ & Mode & Exp. $\omega\left(\mathrm{cm}^{-1}\right)$ \\
\hline $\mathrm{E}(\mathrm{RI})$ & 68.414 & $\mathrm{E}(\mathrm{RI})$ & 64.4806 & & \\
\hline A2(I) & 81.590 & A2(I) & 78.057 & & \\
\hline $\mathrm{E}(\mathrm{RI})$ & 90.897 & $\mathrm{E}(\mathrm{RI})$ & 87.931 & & \\
\hline A2(I) & 104.940 & A2(I) & 106.544 & & \\
\hline $\mathrm{E}(\mathrm{RI})$ & 116.314 & $\mathrm{E}(\mathrm{RI})$ & 107.578 & & \\
\hline $\mathrm{E}(\mathrm{RI})$ & 146.135 & A2(I) & 139.669 & & \\
\hline A2(I) & 147.536 & $\mathrm{E}(\mathrm{RI})$ & 142.2376 & & \\
\hline $\mathrm{E}(\mathrm{RI})$ & 157.944 & $\mathrm{E}(\mathrm{RI})$ & 156.448 & & \\
\hline $\mathrm{E}(\mathrm{RI})$ & 191.967 & $\mathrm{E}(\mathrm{RI})$ & 187.203 & & \\
\hline A2(I) & 205.210 & A2(I) & 195.776 & & \\
\hline $\mathrm{E}(\mathrm{RI})$ & 223.289 & $\mathrm{E}(\mathrm{RI})$ & 214.890 & & \\
\hline $\mathrm{E}(\mathrm{RI})$ & 247.906 & $\mathrm{E}(\mathrm{RI})$ & 240.042 & & \\
\hline A2(I) & 251.242 & A2(I) & 241.844 & & \\
\hline $\mathrm{E}(\mathrm{RI})$ & 355.281 & $\mathrm{E}(\mathrm{RI})$ & 351.190 & & \\
\hline $\mathrm{E}(\mathrm{RI})$ & 464.691 & $\mathrm{E}(\mathrm{RI})$ & 456.668 & $\mathrm{E}(\mathrm{RI})$ & $443 b$ \\
\hline $\mathrm{E}(\mathrm{RI})$ & 508.088 & $\mathrm{E}(\mathrm{RI})$ & 503.035 & & \\
\hline A2(I) & 533.472 & A2(I) & 526.886 & A2(I) & 539s \\
\hline $\mathrm{E}(\mathrm{RI})$ & 554.754 & $\mathrm{E}(\mathrm{RI})$ & 548.035 & $\mathrm{E}(\mathrm{RI})$ & 553w \\
\hline A2(I) & 570.131 & A2(I) & 563.846 & A2(I) & $560 w$ \\
\hline $\mathrm{E}(\mathrm{RI})$ & 575.401 & $\mathrm{E}(\mathrm{RI})$ & 568.750 & $\mathrm{E}(\mathrm{RI})$ & $594 s$ \\
\hline $\mathrm{E}(\mathrm{RI})$ & 944.692 & $\mathrm{E}(\mathrm{RI})$ & 928.447 & & \\
\hline $\mathrm{E}(\mathrm{RI})$ & 964.640 & $\mathrm{E}(\mathrm{RI})$ & 946.861 & $\mathrm{E}(\mathrm{RI})$ & $949 s$ \\
\hline A2(I) & 982.452 & A2(I) & 964.907 & & \\
\hline $\mathrm{E}(\mathrm{RI})$ & 985.354 & $\mathrm{E}(\mathrm{RI})$ & 968.176 & $\mathrm{E}(\mathrm{RI})$ & $957 \mathrm{~s}$ \\
\hline A2(I) & 1017.977 & A2(I) & 996.597 & & \\
\hline \multirow[t]{2}{*}{$\mathrm{E}(\mathrm{RI})$} & 1068.679 & $\mathrm{E}(\mathrm{RI})$ & 1047.101 & $\mathrm{E}(\mathrm{RI})$ & $1023 \mathrm{~s}$ \\
\hline & & & & & $1065 \mathrm{~s}$ \\
\hline
\end{tabular}


(b). Monazite-type ( $\left.\mathrm{BiPO}_{4}-\mathrm{II}\right)$

\begin{tabular}{|c|c|c|c|c|c|}
\hline Mode & LDA $\omega\left(\mathrm{cm}^{-1}\right)$ & Mode & PBESol $\omega\left(\mathrm{cm}^{-1}\right)$ & Mode & Exp. $\omega\left(\mathrm{cm}^{-1}\right)$ \\
\hline $\mathrm{Au}(\mathrm{I})$ & 71.850 & $\mathrm{Au}(\mathrm{I})$ & 65.915 & & \\
\hline $\mathrm{Bu}(\mathrm{I})$ & 83.258 & $\mathrm{Bu}(\mathrm{I})$ & 75.555 & & \\
\hline $\mathrm{Au}(\mathrm{I})$ & 86.527 & $\mathrm{Au}(\mathrm{I})$ & 83.395 & & \\
\hline $\mathrm{Bu}(\mathrm{I})$ & 112.812 & $\mathrm{Bu}(\mathrm{I})$ & 102.976 & & \\
\hline $\mathrm{Au}(\mathrm{I})$ & 124.053 & $\mathrm{Au}(\mathrm{I})$ & 112.116 & & \\
\hline $\mathrm{Bu}(\mathrm{I})$ & 154.208 & $\mathrm{Bu}(\mathrm{I})$ & 147.041 & & \\
\hline \multirow[t]{2}{*}{$\mathrm{Au}(\mathrm{I})$} & 157.143 & $\mathrm{Au}(\mathrm{I})$ & 154.447 & & \\
\hline & & $\mathrm{Bu}(\mathrm{I})$ & 172.493 & & \\
\hline $\mathrm{Au}(\mathrm{I})$ & 179.859 & $\mathrm{Au}(\mathrm{I})$ & 174.495 & & \\
\hline $\mathrm{Bu}(\mathrm{I})$ & 180.059 & & & & \\
\hline $\mathrm{Bu}(\mathrm{I})$ & 194.436 & $\mathrm{Bu}(\mathrm{I})$ & 188.305 & & \\
\hline $\mathrm{Au}(\mathrm{I})$ & 208.746 & $\mathrm{Au}(\mathrm{I})$ & 203.716 & & \\
\hline $\mathrm{Bu}(\mathrm{I})$ & 217.652 & $\mathrm{Bu}(\mathrm{I})$ & 213.757 & & \\
\hline \multirow[t]{2}{*}{$\mathrm{Au}(\mathrm{I})$} & 236.999 & $\mathrm{Au}(\mathrm{I})$ & 227.967 & & \\
\hline & & $\mathrm{Au}(\mathrm{I})$ & 254.186 & & \\
\hline $\mathrm{Bu}(\mathrm{I})$ & 260.915 & $\mathrm{Bu}(\mathrm{I})$ & 256.421 & & \\
\hline $\mathrm{Au}(\mathrm{I})$ & 262.483 & & & & \\
\hline $\mathrm{Bu}(\mathrm{I})$ & 366.722 & $\mathrm{Bu}(\mathrm{I})$ & 359.430 & & \\
\hline $\mathrm{Au}(\mathrm{I})$ & 368.957 & $\mathrm{Au}(\mathrm{I})$ & 363.934 & & \\
\hline $\mathrm{Au}(\mathrm{I})$ & 455.618 & $\mathrm{Au}(\mathrm{I})$ & 449.496 & & \\
\hline $\mathrm{Bu}(\mathrm{I})$ & 470.295 & $\mathrm{Bu}(\mathrm{I})$ & 463.373 & $\mathrm{Bu}(\mathrm{I})+\mathrm{Bu}(\mathrm{I})$ & $473 w$ \\
\hline $\mathrm{Au}(\mathrm{I})$ & 502.284 & $\mathrm{Au}(\mathrm{I})$ & 497.932 & & \\
\hline $\mathrm{Bu}(\mathrm{I})$ & 519.996 & $\mathrm{Bu}(\mathrm{I})$ & 514.844 & & \\
\hline $\mathrm{Au}(\mathrm{I})$ & 528.802 & $\mathrm{Au}(\mathrm{I})$ & 523.784 & $\mathrm{Au}(\mathrm{I})$ & $529 \mathrm{~s}$ \\
\hline $\mathrm{Bu}(\mathrm{I})$ & 539.276 & $\mathrm{Bu}(\mathrm{I})$ & 533.091 & $\mathrm{Bu}(\mathrm{I})$ & $554 \mathrm{~s}$ \\
\hline $\mathrm{Bu}(\mathrm{I})$ & 567.996 & $\mathrm{Bu}(\mathrm{I})$ & 562.579 & $\mathrm{Bu}(\mathrm{I})$ & $564 s$ \\
\hline $\mathrm{Au}(\mathrm{I})$ & 583.440 & $\mathrm{Au}(\mathrm{I})$ & 576.756 & $\operatorname{Au}(\mathrm{I})$ & $604 s$ \\
\hline $\mathrm{Bu}(\mathrm{I})$ & 919.141 & $\mathrm{Bu}(\mathrm{I})$ & 898.993 & $\mathrm{Bu}(\mathrm{I})$ & $876 w$ \\
\hline $\mathrm{Au}(\mathrm{I})$ & 927.614 & $\mathrm{Au}(\mathrm{I})$ & 908.867 & & \\
\hline $\mathrm{Au}(\mathrm{I})$ & 937.154 & $\mathrm{Au}(\mathrm{I})$ & 917.306 & & \\
\hline $\mathrm{Bu}(\mathrm{I})$ & 941.690 & $\mathrm{Bu}(\mathrm{I})$ & 922.276 & $\mathrm{Bu}(\mathrm{I})$ & $930 \mathrm{~s}$ \\
\hline $\mathrm{Bu}(\mathrm{I})$ & 975.847 & $\mathrm{Bu}(\mathrm{I})$ & 960.671 & $\mathrm{Bu}(\mathrm{I})$ & $958 s$ \\
\hline $\mathrm{Au}(\mathrm{I})$ & 981.151 & $\mathrm{Au}(\mathrm{I})$ & 963.974 & & \\
\hline $\mathrm{Bu}(\mathrm{I})$ & 1041.426 & $\mathrm{Bu}(\mathrm{I})$ & 1023.317 & $\mathrm{Bu}(\mathrm{I})$ & $1011 \mathrm{~s}$ \\
\hline \multirow[t]{2}{*}{$\operatorname{Au}(\mathrm{I})$} & 1052.968 & $\mathrm{Au}(\mathrm{I})$ & 1033.925 & $\mathrm{Au}(\mathrm{I})$ & $1031 \mathrm{~s}$ \\
\hline & & & & & $1076 \mathrm{~s}$ \\
\hline
\end{tabular}


(c). $\mathrm{SbPO}_{4}$-type $\left(\mathrm{BiPO}_{4}\right.$-III).)

\begin{tabular}{|c|c|c|c|c|c|}
\hline Mode & $\operatorname{LDA} \omega\left(\mathrm{cm}^{-1}\right)$ & Mode & PBESol $\omega\left(\mathrm{cm}^{-1}\right)$ & Mode & $\operatorname{Exp} . \omega\left(\mathrm{cm}^{-1}\right)$ \\
\hline $\mathrm{Bu}(\mathrm{I})$ & 120.184 & $\mathrm{Bu}(\mathrm{I})$ & 109.080 & & \\
\hline $\mathrm{Au}(\mathrm{I})$ & 126.955 & $\mathrm{Au}(\mathrm{I})$ & 113.516 & & \\
\hline $\mathrm{Bu}(\mathrm{I})$ & 153.507 & $\mathrm{Bu}(\mathrm{I})$ & 149.909 & & \\
\hline $\mathrm{Au}(\mathrm{I})$ & 185.196 & $\mathrm{Au}(\mathrm{I})$ & 182.7673 & & \\
\hline $\mathrm{Bu}(\mathrm{I})$ & 201.340 & $\mathrm{Bu}(\mathrm{I})$ & 196.410 & & \\
\hline $\mathrm{Au}(\mathrm{I})$ & 209.880 & $\mathrm{Au}(\mathrm{I})$ & 203.616 & & \\
\hline $\mathrm{Au}(\mathrm{I})$ & 322.725 & $\mathrm{Au}(\mathrm{I})$ & 320.468 & & \\
\hline & & & & & $\begin{array}{l}421 w \\
434 w \\
451 w \\
457 w\end{array}$ \\
\hline $\mathrm{Bu}(\mathrm{I})$ & 481.869 & $\mathrm{Bu}(\mathrm{I})$ & 474.848 & $\mathrm{Bu}(\mathrm{I})$ & $472 w$ \\
\hline $\mathrm{Au}(\mathrm{I})$ & 494.545 & $\mathrm{Au}(\mathrm{I})$ & 491.860 & $\mathrm{Au}(\mathrm{I})$ & $494 s$ \\
\hline $\mathrm{Bu}(\mathrm{I})$ & 508.088 & $\mathrm{Bu}(\mathrm{I})$ & 503.702 & $\mathrm{Bu}(\mathrm{I})$ & $\begin{array}{l}513 \mathrm{~s} \\
527 \mathrm{w}\end{array}$ \\
\hline $\mathrm{Bu}(\mathrm{I})$ & 582.373 & $\mathrm{Bu}(\mathrm{I})$ & 576.222 & $\mathrm{Bu}(\mathrm{I})$ & $\begin{array}{l}554 \mathrm{~s} \\
630 \mathrm{~s}\end{array}$ \\
\hline $\mathrm{Au}(\mathrm{I})$ & 933.651 & $\mathrm{Au}(\mathrm{I})$ & 913.670 & & \\
\hline $\mathrm{Bu}(\mathrm{I})$ & 936.120 & $\mathrm{Bu}(\mathrm{I})$ & 918.707 & $\begin{array}{l}\mathrm{Bu}(\mathrm{I}) \\
\mathrm{Bu}(\mathrm{I})\end{array}$ & $\begin{array}{l}928 w \\
963 s\end{array}$ \\
\hline $\mathrm{Bu}(\mathrm{I})$ & 1003.333 & $\mathrm{Bu}(\mathrm{I})$ & 987.624 & $\mathrm{Bu}(\mathrm{I})$ & $1005 s$ \\
\hline $\mathrm{Bu}(\mathrm{I})$ & 1048.631 & $\mathrm{Bu}(\mathrm{I})$ & 1029.521 & $\mathrm{Bu}(\mathrm{I})$ & $\begin{array}{l}1029 s \\
1105 s\end{array}$ \\
\hline
\end{tabular}


Table 5: Calculated and observed Raman modes of $\mathrm{BiPO}_{4}$ phases. ((a). Trigonal (I), (b). Monazite-type (II) and (c). SbPO 4 -type (III)).

(a). Trigonal $\left(\mathrm{BiPO}_{4}-\mathrm{I}\right)$,

\begin{tabular}{|c|c|c|c|c|c|}
\hline Mode & LDA - $\omega\left(\mathrm{cm}^{-1}\right)$ & Mode & PBESol $\omega\left(\mathrm{cm}^{-1}\right)$ & Modes & Exper. - $\omega\left(\mathrm{cm}^{-1}\right)$ \\
\hline $\mathrm{E}(\mathrm{RI})$ & 68.414 & $\mathrm{E}(\mathrm{RI})$ & 64.480 & & \\
\hline A1(R) & 79.922 & $\mathrm{~A} 1(\mathrm{R})$ & 77.223 & & \\
\hline $\mathrm{E}(\mathrm{RI})$ & 90.897 & $\mathrm{E}(\mathrm{RI})$ & 87.931 & $\mathrm{E}(\mathrm{RI})$ & 93.6 \\
\hline $\mathrm{E}(\mathrm{RI})$ & 116.314 & $\mathrm{E}(\mathrm{RI})$ & 107.578 & & \\
\hline A1(R) & 117.148 & $\mathrm{~A} 1(\mathrm{R})$ & 109.780 & $\mathrm{~A} 1(\mathrm{R})$ & 118.7 \\
\hline $\mathrm{E}(\mathrm{RI})$ & 146.135 & $\mathrm{E}(\mathrm{RI})$ & 142.237 & $\mathrm{E}(\mathrm{RI})$ & 146.9 \\
\hline $\mathrm{E}(\mathrm{RI})$ & 157.944 & $\mathrm{E}(\mathrm{RI})$ & 156.448 & $\mathrm{E}(\mathrm{RI})$ & 162.2 \\
\hline $\mathrm{E}(\mathrm{RI})$ & 191.967 & $\mathrm{E}(\mathrm{RI})$ & 187.203 & $\mathrm{E}(\mathrm{RI})$ & 192.6 \\
\hline A1(R) & 195.303 & $\mathrm{~A} 1(\mathrm{R})$ & 191.273 & & \\
\hline $\mathrm{E}(\mathrm{RI})$ & 223.289 & $\mathrm{E}(\mathrm{RI})$ & 214.890 & $\mathrm{E}(\mathrm{RI})$ & 220.8 \\
\hline $\mathrm{E}(\mathrm{RI})$ & 247.906 & $\mathrm{E}(\mathrm{RI})$ & 240.042 & $\mathrm{E}(\mathrm{RI})$ & 241.7 \\
\hline $\mathrm{E}(\mathrm{RI})$ & 355.281 & $\mathrm{E}(\mathrm{RI})$ & 351.190 & $\mathrm{E}(\mathrm{RI})$ & 367.9 \\
\hline A1(R) & 373.494 & $\mathrm{~A} 1(\mathrm{R})$ & 370.705 & $\mathrm{~A} 1(\mathrm{R})$ & 378.8 \\
\hline A1(R) & 438.506 & $\mathrm{~A} 1(\mathrm{R})$ & 430.515 & $\mathrm{~A} 1(\mathrm{R})$ & 452.8 \\
\hline $\mathrm{E}(\mathrm{RI})$ & 464.691 & $\mathrm{E}(\mathrm{RI})$ & 456.668 & $\mathrm{E}(\mathrm{RI})$ & 464.7 \\
\hline $\mathrm{E}(\mathrm{RI})$ & 508.088 & $\mathrm{E}(\mathrm{RI})$ & 503.035 & $\mathrm{E}(\mathrm{RI})$ & 497.5 \\
\hline A1(R) & 532.938 & $\mathrm{~A} 1(\mathrm{R})$ & 527.186 & $\mathrm{~A} 1(\mathrm{R})$ & 546.4 \\
\hline $\mathrm{E}(\mathrm{RI})$ & 554.754 & $\mathrm{E}(\mathrm{RI})$ & 548.035 & $\mathrm{E}(\mathrm{RI})$ & 579 \\
\hline $\mathrm{E}(\mathrm{RI})$ & 575.401 & $\mathrm{E}(\mathrm{RI})$ & 568.750 & $\mathrm{E}(\mathrm{RI})$ & 606.2 \\
\hline $\mathrm{E}(\mathrm{RI})$ & 944.692 & $\mathrm{E}(\mathrm{RI})$ & 928.447 & $\mathrm{E}(\mathrm{RI})$ & 943.7 \\
\hline A1(R) & 949.162 & A1(R) & 933.217 & & \\
\hline $\mathrm{E}(\mathrm{RI})$ & 964.640 & $\mathrm{E}(\mathrm{RI})$ & 946.861 & $\mathrm{E}(\mathrm{RI})$ & 968.8 \\
\hline A1(R) & 975.881 & A1(R) & 957.735 & $\mathrm{~A} 1(\mathrm{R})$ & 979.7 \\
\hline $\mathrm{E}(\mathrm{RI})$ & 985.354 & $\mathrm{E}(\mathrm{RI})$ & 968.176 & $\mathrm{E}(\mathrm{RI})$ & 1012 \\
\hline $\mathrm{E}(\mathrm{RI})$ & 1068.679 & $\mathrm{E}(\mathrm{RI})$ & 1047.101 & $\mathrm{E}(\mathrm{RI})$ & 1073.4 \\
\hline
\end{tabular}


(b). Monazite-type $\left(\mathrm{BiPO}_{4}-\mathrm{II}\right)$

\begin{tabular}{|c|c|c|c|c|c|}
\hline Mode & $\operatorname{LDA} \omega\left(\mathrm{cm}^{-1}\right)$ & Mode & PBESol $\omega\left(\mathrm{cm}^{-1}\right)$ & Mode & Exper $\omega\left(\mathrm{cm}^{-1}\right)$ \\
\hline $\operatorname{Ag}(\mathrm{R})$ & 64.378 & $\operatorname{Ag}(\mathrm{R})$ & 58.209 & $\mathrm{Ag}$ & 50,9 \\
\hline $\operatorname{Ag}(R)$ & 74.285 & $\operatorname{Ag}(\mathrm{R})$ & 68.383 & $\mathrm{Ag}$ & 59,8 \\
\hline $\mathrm{Bg}(\mathrm{R})$ & 75.286 & $\mathrm{Bg}(\mathrm{R})$ & 73.287 & $\mathrm{Bg}$ & 69,9 \\
\hline $\mathrm{Bg}(\mathrm{R})$ & 93.699 & $\mathrm{Bg}(\mathrm{R})$ & 89.332 & $\mathrm{Bg}$ & 89,6 \\
\hline $\operatorname{Ag}(R)$ & 97.335 & $\operatorname{Ag}(\mathrm{R})$ & 95.337 & Ag & 97,3 \\
\hline \multirow[t]{2}{*}{$\mathrm{Bg}(\mathrm{R})$} & 106.908 & $\mathrm{Bg}(\mathrm{R})$ & 102.642 & $\mathrm{Bg}$ & 108,6 \\
\hline & & $\operatorname{Ag}(R)$ & 132.697 & $\mathrm{Bg}$ & 131,1 \\
\hline $\mathrm{Bg}(\mathrm{R})$ & 134.561 & $\mathrm{Bg}(\mathrm{R})$ & 134.732 & $\mathrm{Bg}$ & 135,5 \\
\hline $\operatorname{Ag}(R)$ & 138.597 & & & & \\
\hline $\operatorname{Ag}(\mathrm{R})$ & 166.950 & $\operatorname{Ag}(\mathrm{R})$ & 165.688 & $\mathrm{Ag}$ & 169,9 \\
\hline $\mathrm{Bg}(\mathrm{R})$ & 172.921 & $\mathrm{Bg}(\mathrm{R})$ & 167.790 & $\mathrm{Bg}+\mathrm{Ag}$ & 176,7 \\
\hline $\operatorname{Ag}(R)$ & 172.787 & $\operatorname{Ag}(\mathrm{R})$ & 168.424 & & \\
\hline \multirow[t]{2}{*}{$A g(R)+B g(R)$} & 192.601 & $\operatorname{Ag}(\mathrm{R})$ & 184.002 & $\mathrm{Ag}+\mathrm{Bg}$ & 182,7 \\
\hline & & $\mathrm{Bg}(\mathrm{R})$ & 185.236 & & \\
\hline $\mathrm{Bg}(\mathrm{R})$ & 212.115 & $\mathrm{Bg}(\mathrm{R})$ & 203.883 & $\mathrm{Bg}$ & 207,4 \\
\hline $\mathrm{Bg}(\mathrm{R})$ & 235.931 & $\operatorname{Bg}(\mathrm{R})$ & 226.199 & $\mathrm{Bg}$ & 229,8 \\
\hline $\operatorname{Ag}(\mathrm{R})$ & 240.434 & $\operatorname{Ag}(\mathrm{R})$ & 231.903 & $\mathrm{Ag}$ & 237,1 \\
\hline $\mathrm{Bg}(\mathrm{R})$ & 279.095 & $\mathrm{Bg}(\mathrm{R})$ & 271.332 & $\mathrm{Bg}$ & 272,5 \\
\hline $\mathrm{Ag}(\mathrm{R})$ & 286.133 & $\operatorname{Ag}(\mathrm{R})$ & 278.671 & $\mathrm{Ag}$ & 283,5 \\
\hline $\mathrm{Bg}(\mathrm{R})$ & 375.729 & $\operatorname{Bg}(\mathrm{R})$ & 368.303 & Bg & 388,4 \\
\hline $\operatorname{Ag}(R)$ & 390.439 & $\operatorname{Ag}(\mathrm{R})$ & 384.549 & $\mathrm{Ag}$ & 407,4 \\
\hline $\mathrm{Ag}(\mathrm{R})$ & 445.077 & $\operatorname{Ag}(\mathrm{R})$ & 438.688 & $\mathrm{Ag}$ & 457,0 \\
\hline $\mathrm{Bg}(\mathrm{R})$ & 477.833 & $\operatorname{Bg}(\mathrm{R})$ & 469.945 & $\mathrm{Bg}$ & 463,5 \\
\hline $\mathrm{Ag}(\mathrm{R})$ & 503.051 & $\operatorname{Ag}(\mathrm{R})$ & 497.999 & Ag & 495,9 \\
\hline $\operatorname{Ag}(R)$ & 531.404 & $\operatorname{Ag}(\mathrm{R})$ & 527.220 & $\mathrm{Ag}$ & 522,7 \\
\hline $\mathrm{Bg}(\mathrm{R})$ & 532.638 & $\mathrm{Bg}(\mathrm{R})$ & 527.287 & $\mathrm{Bg}$ & 557,1 \\
\hline $\mathrm{Bg}(\mathrm{R})$ & 547.749 & $\mathrm{Bg}(\mathrm{R})$ & 540.930 & $\mathrm{Bg}$ & 572,6 \\
\hline $\operatorname{Ag}(\mathrm{R})$ & 573.867 & $\operatorname{Ag}(\mathrm{R})$ & 568.083 & $\mathrm{Ag}$ & 598,3 \\
\hline $\mathrm{Bg}(\mathrm{R})$ & 580.405 & $\mathrm{Bg}(\mathrm{R})$ & 573.921 & $\mathrm{Bg}$ & 604,5 \\
\hline $\mathrm{Ag}(\mathrm{R})$ & 933.184 & $\operatorname{Ag}(\mathrm{R})$ & 911.936 & $\mathrm{Ag}+\mathrm{Bg}$ & 926,3 \\
\hline $\mathrm{Bg}(\mathrm{R})$ & 933.685 & $\mathrm{Bg}(\mathrm{R})$ & 915.772 & & \\
\hline $\operatorname{Ag}(\mathrm{R})$ & 954.933 & $\operatorname{Ag}(\mathrm{R})$ & 935.286 & $\mathrm{Ag}$ & 947,8 \\
\hline $\operatorname{Ag}(\mathrm{R})$ & 973.412 & $\operatorname{Ag}(\mathrm{R})$ & 956.502 & $\mathrm{Ag}$ & 969,5 \\
\hline $\mathrm{Bg}(\mathrm{R})$ & 982,552 & $\mathrm{Bg}(\mathrm{R})$ & 962.272 & $\mathrm{Bg}$ & 981,0 \\
\hline $\mathrm{Bg}(\mathrm{R})$ & 1001.799 & $\mathrm{Bg}(\mathrm{R})$ & 985.256 & $\mathrm{Bg}$ & 1021,0 \\
\hline $\operatorname{Ag}(\mathrm{R})$ & 1027.383 & $\operatorname{Ag}(R)$ & 1010.141 & $\mathrm{Ag}$ & 1039,4 \\
\hline $\mathrm{Bg}(\mathrm{R})$ & 1035.389 & $\mathrm{Bg}(\mathrm{R})$ & 1016.612 & $\mathrm{Bg}$ & 1050,2 \\
\hline
\end{tabular}


(c). $\mathrm{SbPO}_{4}$-type $\left(\mathrm{BiPO}_{4}\right.$-III).)

\begin{tabular}{|c|c|c|c|c|c|}
\hline Mode & $\begin{array}{c}\text { LDA } \\
\omega\left(\mathrm{cm}^{-1}\right)\end{array}$ & Mode & $\begin{array}{l}\text { PBESol } \\
\omega\left(\mathrm{cm}^{-1}\right)\end{array}$ & Modes & $\begin{array}{l}\text { Exper. } \\
\omega\left(\mathrm{cm}^{-1}\right)\end{array}$ \\
\hline $\operatorname{Ag}(\mathrm{R})$ & 37.793 & $\mathrm{Ag}(\mathrm{R})$ & 38.294 & $\operatorname{Ag}(\mathrm{R})$ & 56.2 \\
\hline $\mathrm{Ag}(\mathrm{R})$ & 72.050 & $\operatorname{Ag}(\mathrm{R})$ & 66.315 & $\mathrm{Ag}(\mathrm{R})$ & 68.8 \\
\hline $\mathrm{Bg}(\mathrm{R})$ & 108.843 & $\mathrm{Bg}(\mathrm{R})$ & 103.842 & $\mathrm{Bg}(\mathrm{R})$ & 91.7 \\
\hline $\mathrm{Ag}(\mathrm{R})$ & 129.424 & $\mathrm{Ag}(\mathrm{R})$ & 124.624 & & \\
\hline $\mathrm{Bg}(\mathrm{R})$ & 138.763 & $\mathrm{Ag}(\mathrm{R})$ & 132.830 & $\operatorname{Ag}(\mathrm{R})$ & 135.7 \\
\hline $\mathrm{Ag}(\mathrm{R})$ & 140.465 & $\mathrm{Bg}(\mathrm{R})$ & 139.835 & $\mathrm{Bg}(\mathrm{R})$ & 144.5 \\
\hline $\mathrm{Bg}(\mathrm{R})$ & 171.553 & $\mathrm{Bg}(\mathrm{R})$ & 166.922 & $\mathrm{Bg}(\mathrm{R})$ & 171.2 \\
\hline $\mathrm{Ag}(\mathrm{R})$ & 200.240 & $\mathrm{Ag}(\mathrm{R})$ & 195.710 & $\mathrm{Ag}(\mathrm{R})$ & 214.2 \\
\hline $\mathrm{Bg}(\mathrm{R})$ & 267.420 & $\mathrm{Bg}(\mathrm{R})$ & 258.022 & $\mathrm{Bg}(\mathrm{R})$ & 244 \\
\hline $\mathrm{Bg}(\mathrm{R})$ & 326.328 & $\mathrm{Bg}(\mathrm{R})$ & 322.936 & $\mathrm{Bg}(\mathrm{R})$ & 353.6 \\
\hline $\mathrm{Ag}(\mathrm{R})$ & 470.762 & $\mathrm{Ag}(\mathrm{R})$ & 463.573 & $\mathrm{Ag}(\mathrm{R})$ & 486.4 \\
\hline $\mathrm{Ag}(\mathrm{R})$ & 508.221 & $\operatorname{Ag}(\mathrm{R})$ & 504.403 & $\mathrm{Ag}(\mathrm{R})$ & 548.3 \\
\hline $\mathrm{Bg}(\mathrm{R})$ & 539.310 & $\mathrm{Bg}(\mathrm{R})$ & 533.724 & $\mathrm{Bg}(\mathrm{R})$ & 557.1 \\
\hline $\mathrm{Ag}(\mathrm{R})$ & 582.139 & $\mathrm{Ag}(\mathrm{R})$ & 576.856 & $\mathrm{Ag}(\mathrm{R})$ & 609.9 \\
\hline $\mathrm{Ag}(\mathrm{R})$ & 946.694 & $\mathrm{Ag}(\mathrm{R})$ & 929.982 & $\mathrm{Ag}(\mathrm{R})$ & 966.1 \\
\hline $\mathrm{Bg}(\mathrm{R})$ & 955.200 & $\mathrm{Bg}(\mathrm{R})$ & 936.019 & $\mathrm{Bg}(\mathrm{R})$ & 982.9 \\
\hline $\mathrm{Ag}(\mathrm{R})$ & 994.327 & $\mathrm{Ag}(\mathrm{R})$ & 978.050 & $\mathrm{Ag}(\mathrm{R})$ & 1037.8 \\
\hline $\mathrm{Ag}(\mathrm{R})$ & 1035.589 & $\mathrm{Ag}(\mathrm{R})$ & 1017.946 & $\mathrm{Ag}(\mathrm{R})$ & 1046 \\
\hline
\end{tabular}




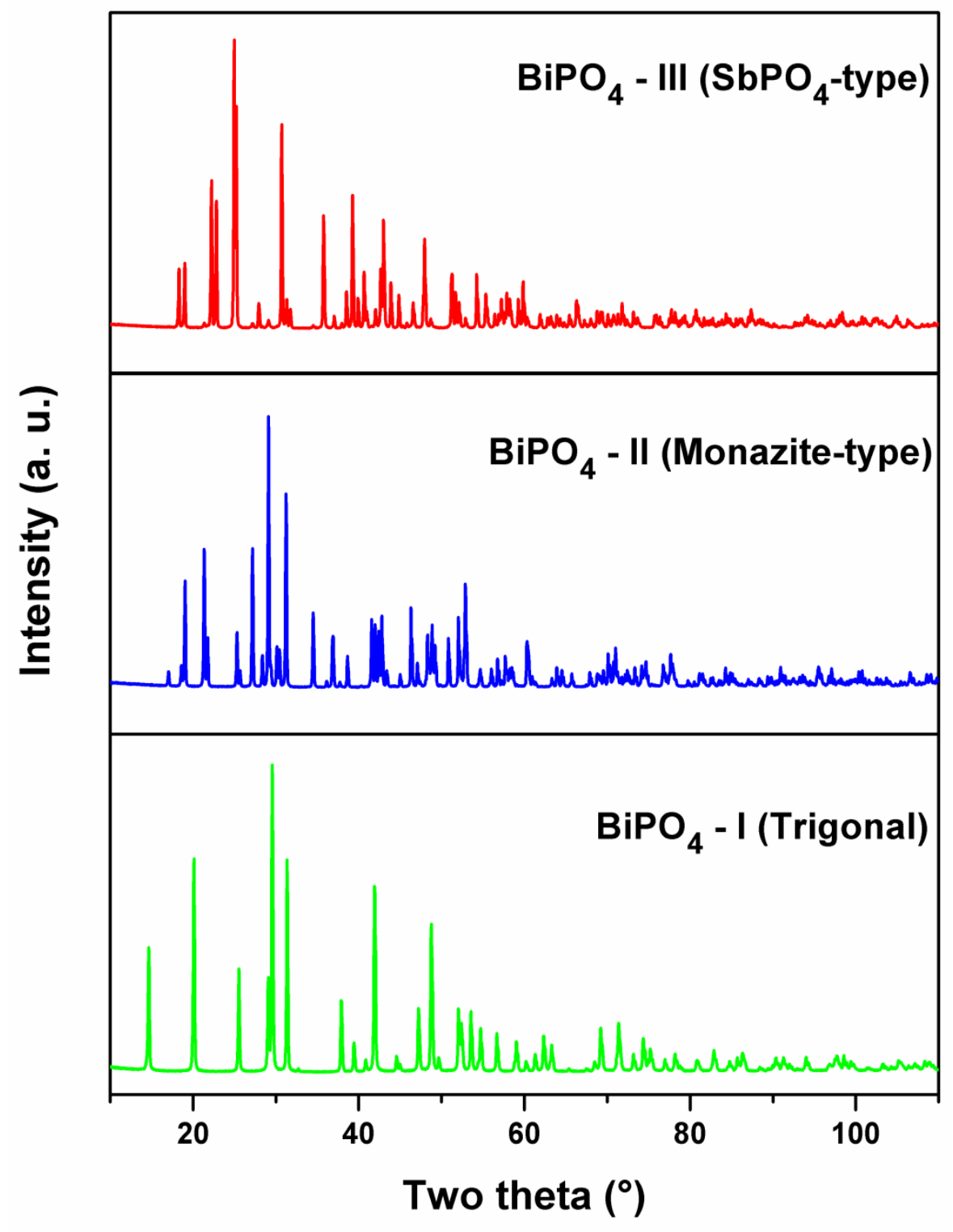

Fig. 1: Powder XRD patters of different phases of $\mathrm{BiPO}_{4}$ at ambient temperature $(\lambda=1.5417 \AA)$. 

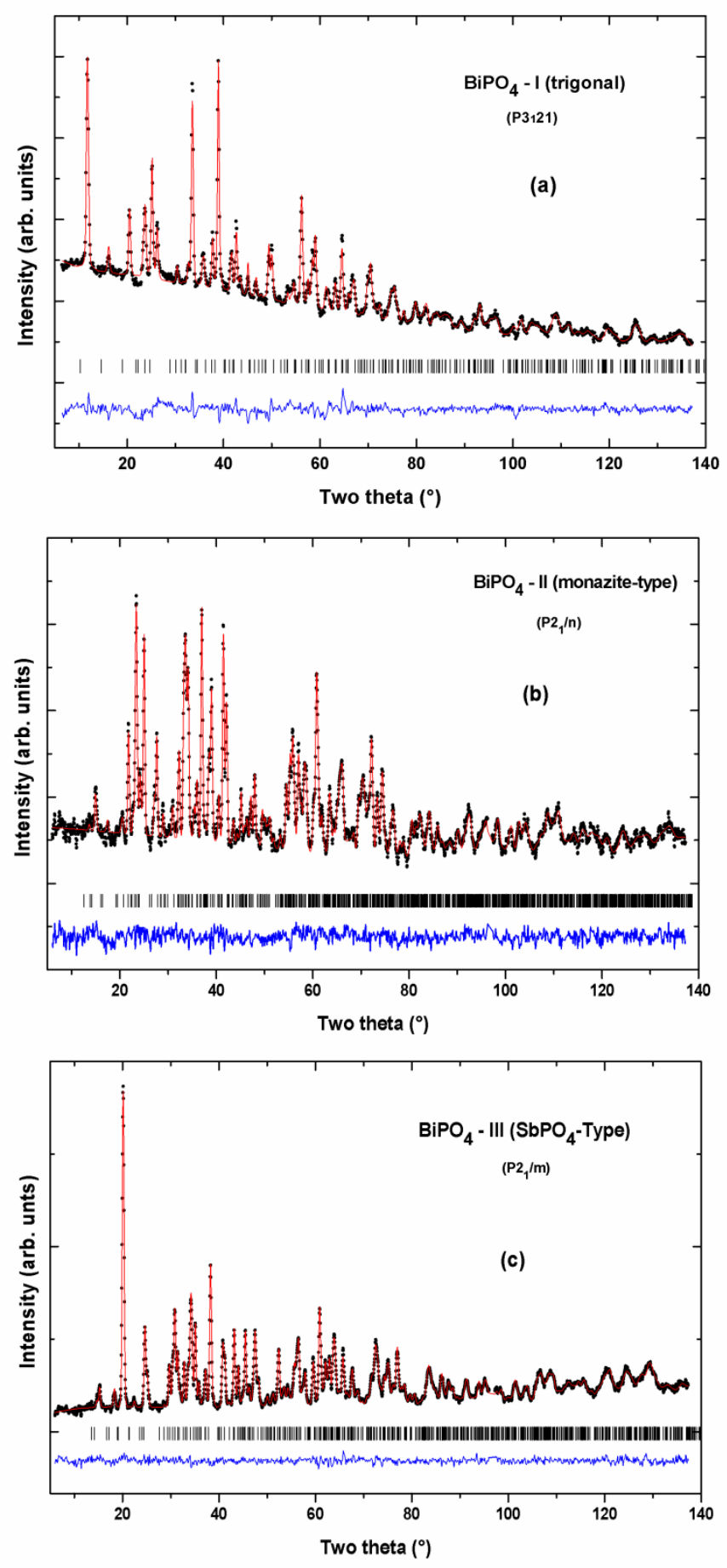

Fig. 2: Rietveld refinement plots of powder neutron diffraction data of $\mathrm{BiPO}_{4}$ phases, (a). Trigonal (I), (b). Monazite-type (II) and (c). SbPO 4 -type (III). ( $\lambda=1.249 \AA$ ). 


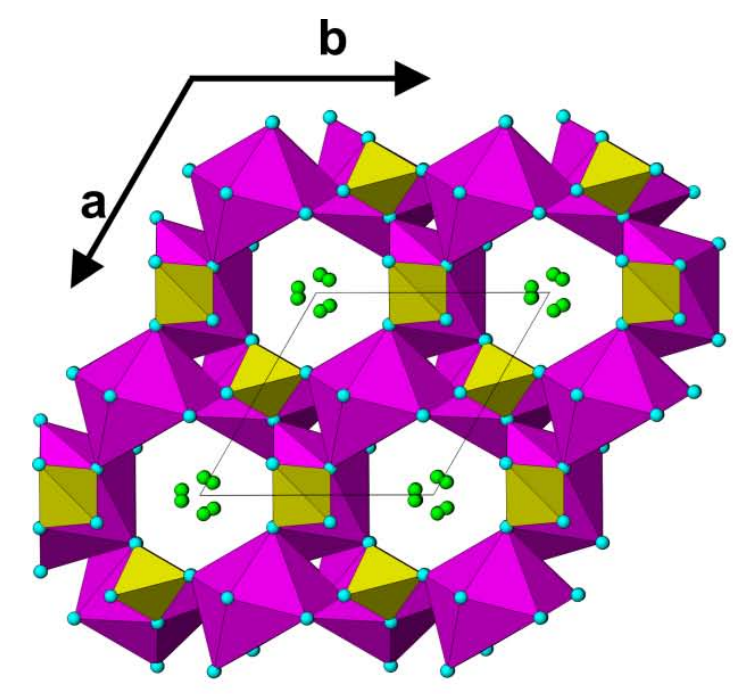

( a )

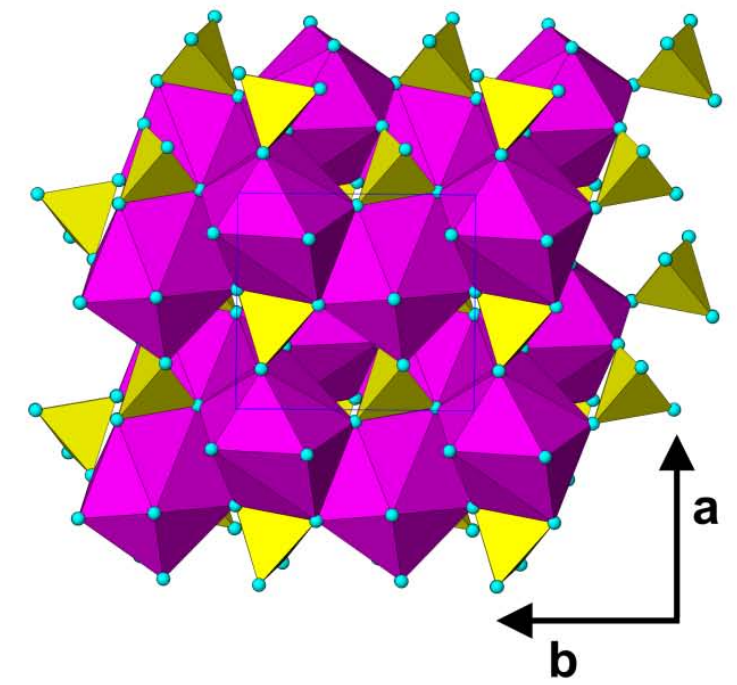

(b)

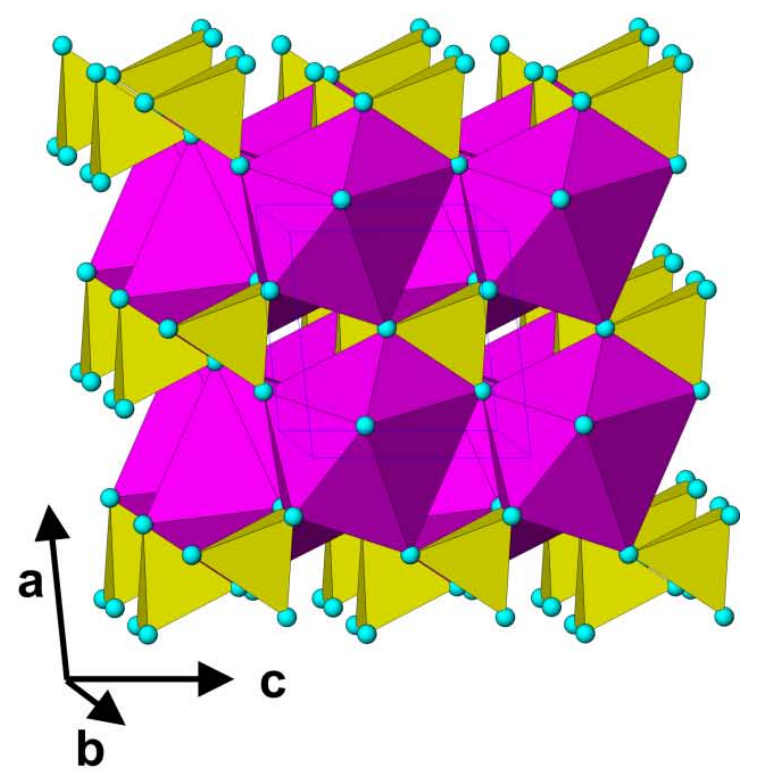

(c)

Fig. 3: Crystal structure of $\mathrm{BiPO}_{4}$ phases, (a). Trigonal (I), (b). Monazite-type (II) and (c). $\mathrm{SbPO}_{4}$-type (III). (BiO 8 (in a and c), BiO9 (in b) and $\mathrm{PO}_{4}$ units are shown). (isolated spheres are $\mathrm{O} 3$ (of water molecules in $\mathrm{BiPO}_{4}-\mathrm{I}$ ). 


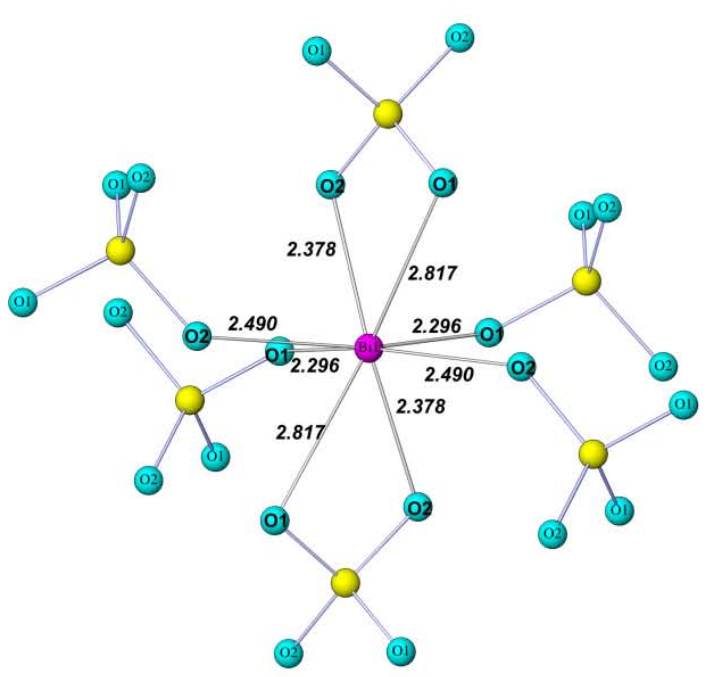

(a)

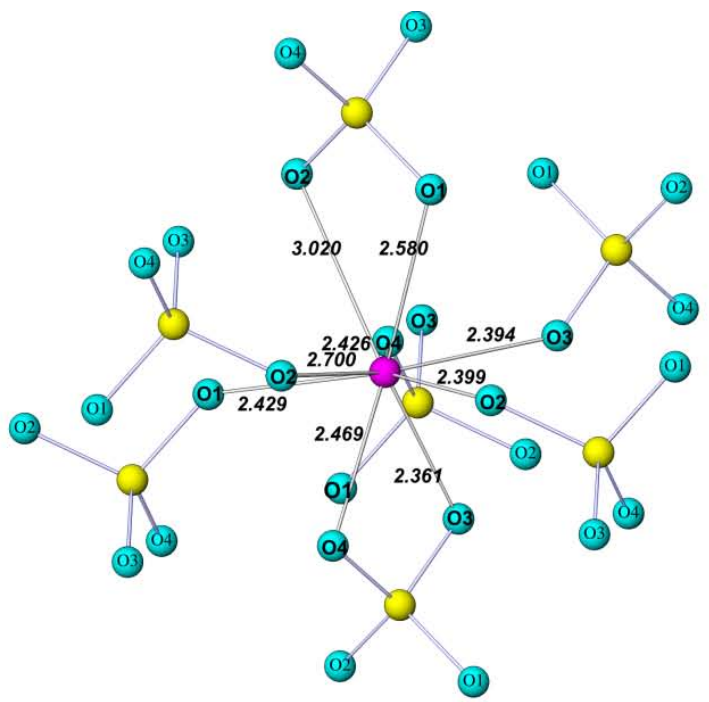

(b)

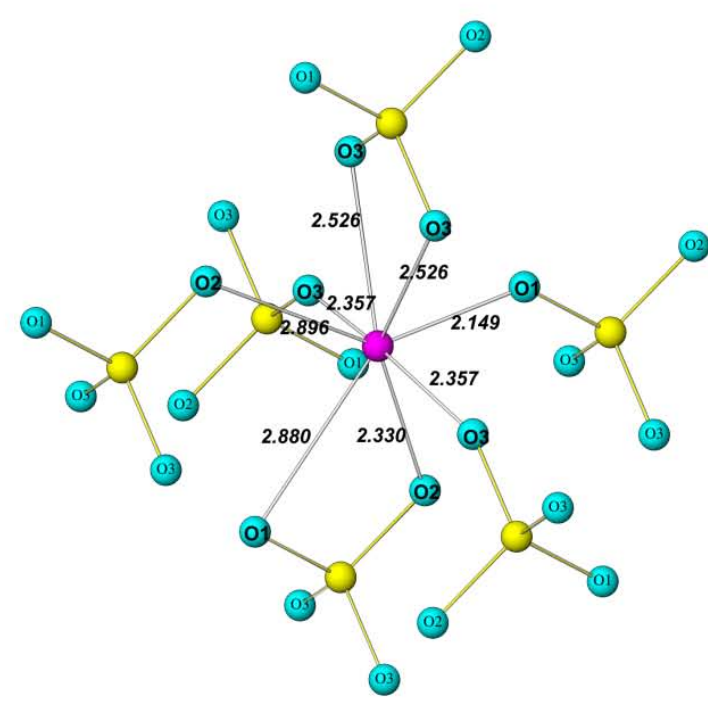

(c)

Fig. 4: Typical coordination around $\mathrm{Bi}^{3+}$ in $\mathrm{BiPO}_{4}$ phases, (a). Trigonal (I), (b). Monazite type (II) and (c). SbPO 4 type (III) 

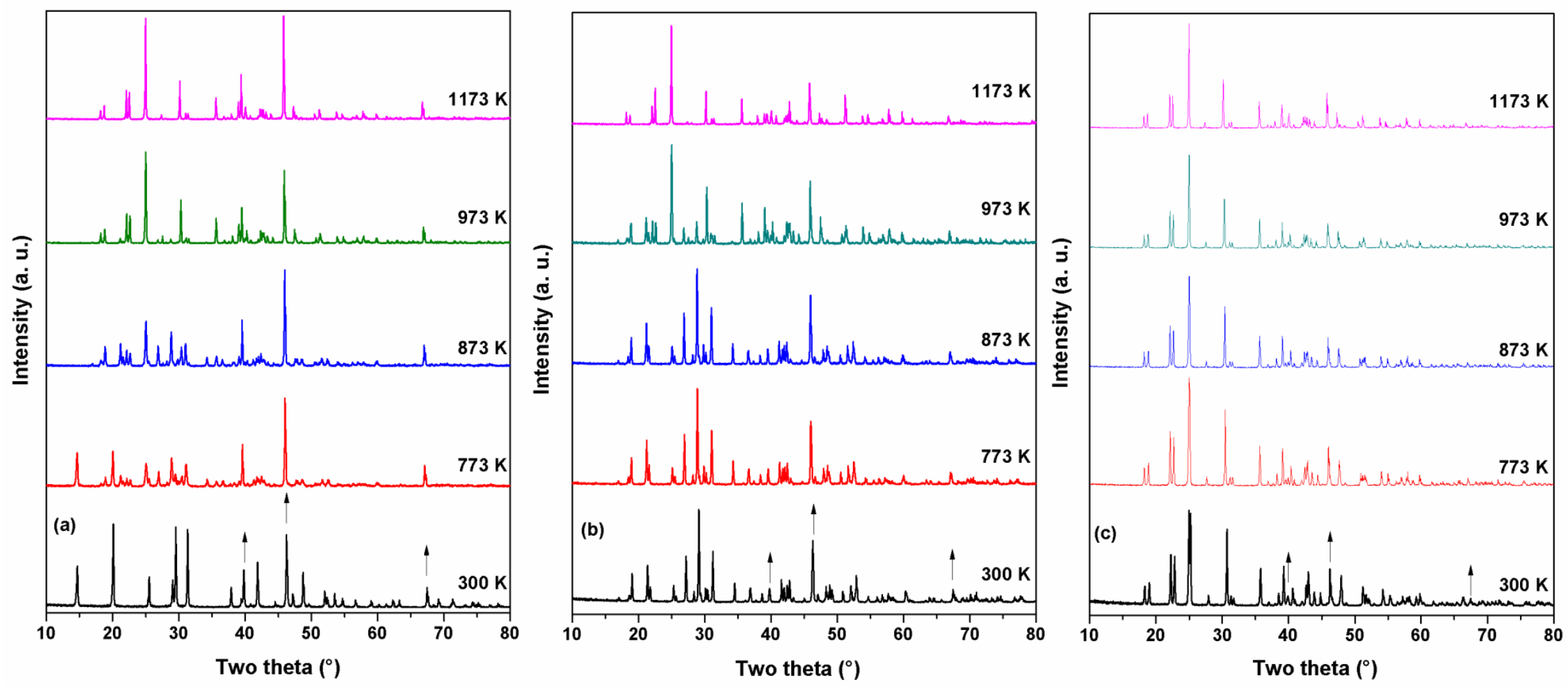

Fig. 5: Powder XRD patterns of $\mathrm{BiPO}_{4}$ phases at representative temperatures. (a). Trigonal (I), (b). Monazite-type (II) and (c). SbPO ${ }_{4-}$ type (III) ( $\uparrow$ indicate platinum sample holder base peaks) 


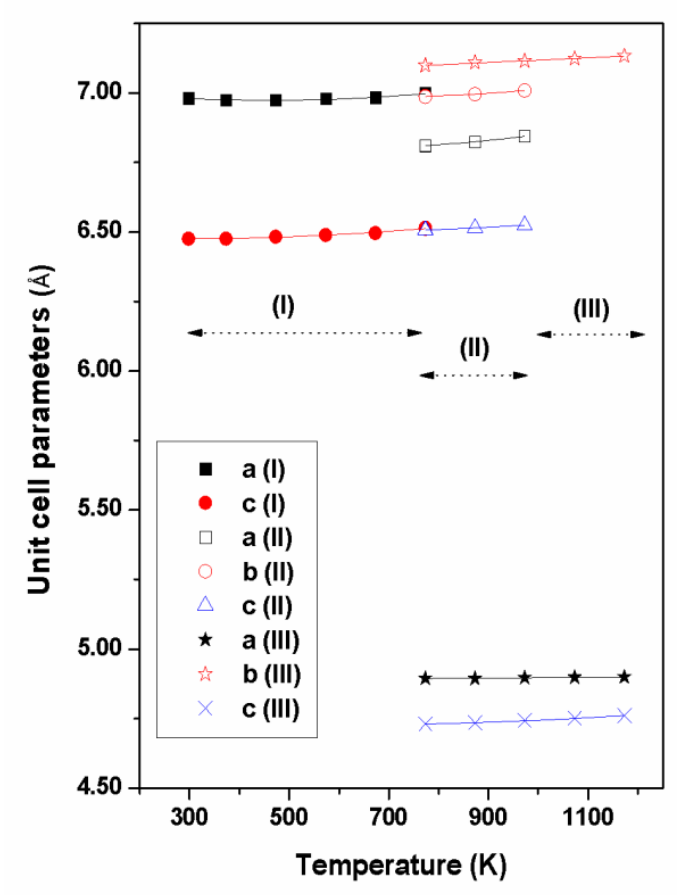

(a)

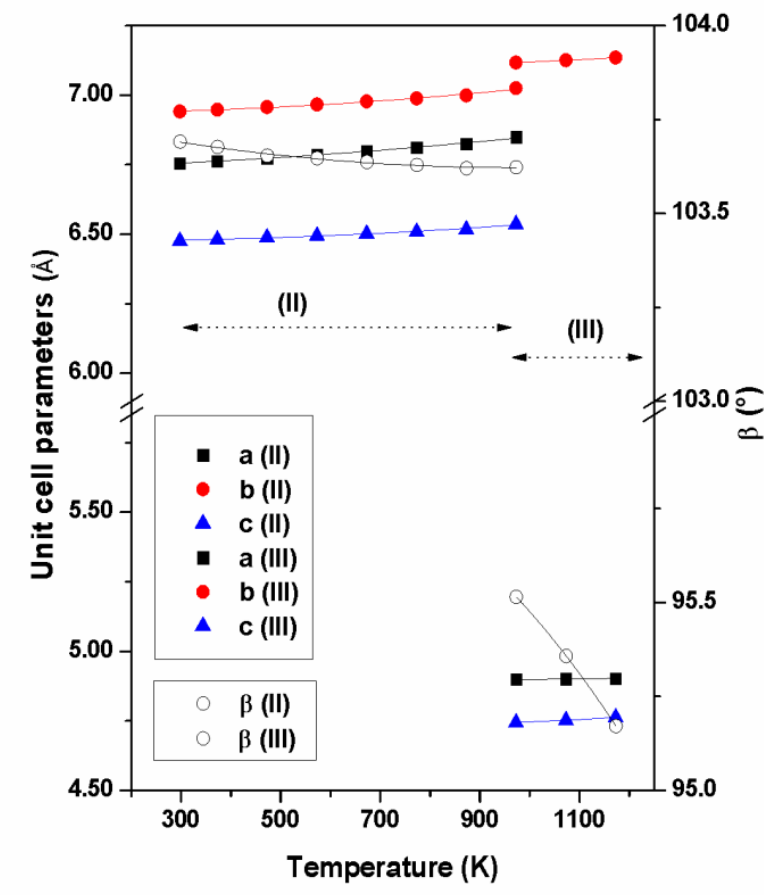

(b)

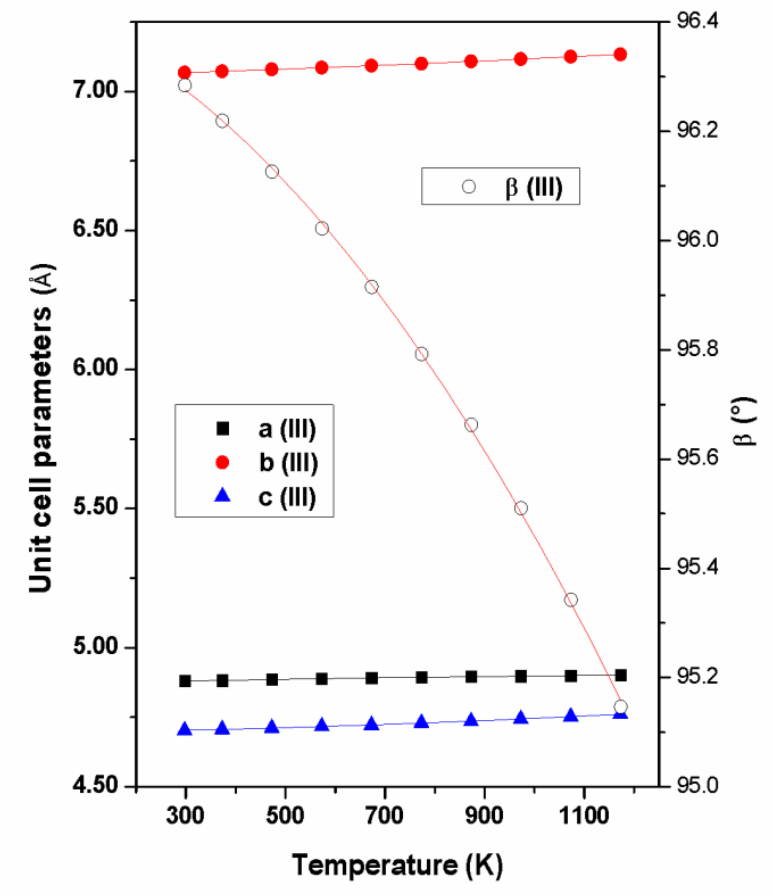

(c)

Fig. 6: Variation of unit cell parameters of $\mathrm{BiPO}_{4}$ with temperature. (a). Trigonal (I), (b). Monazite-type (II) and (c). SbPO 4 -type (III). 


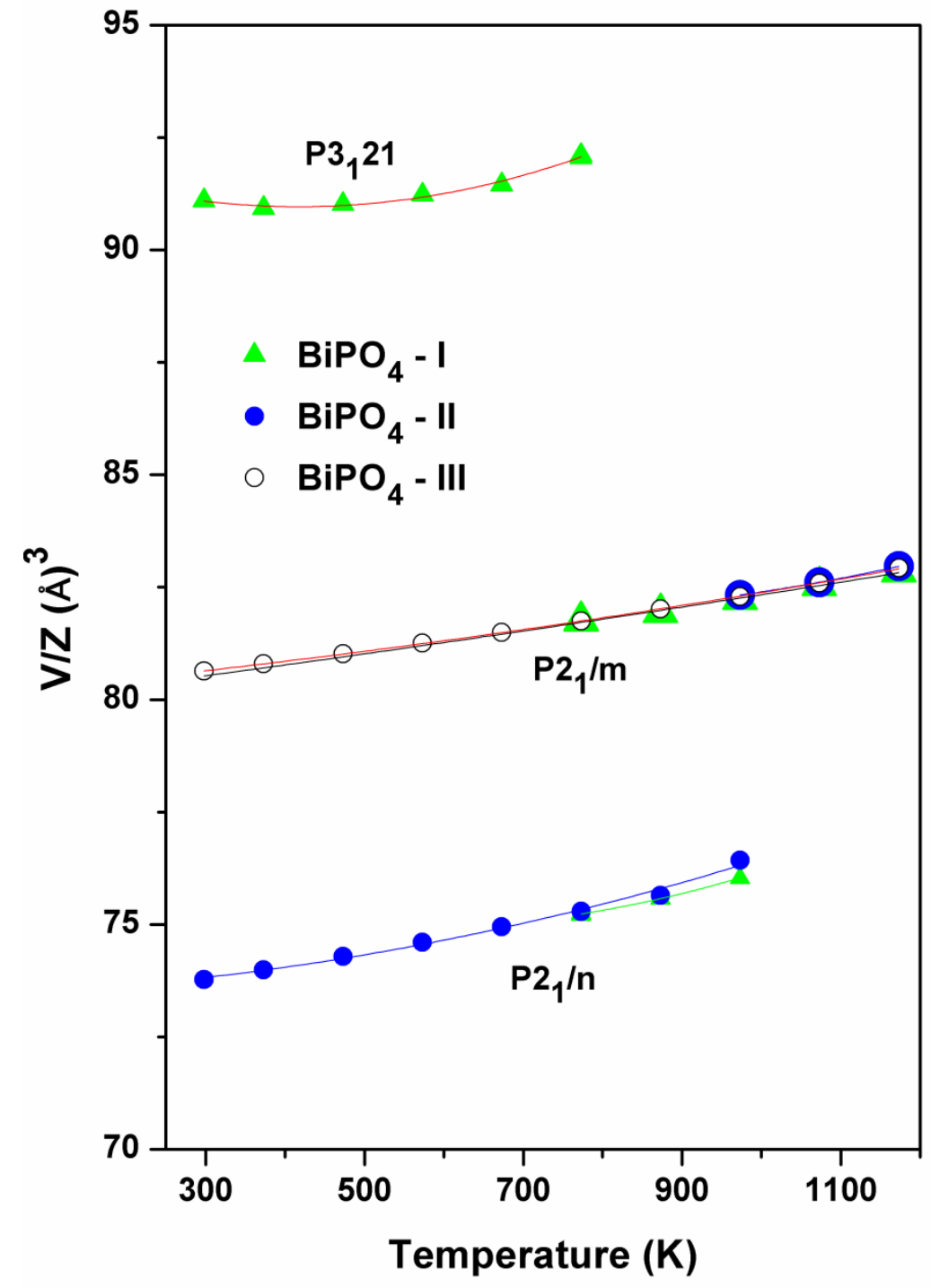

Fig. 7: Variation of molar volume of $\mathrm{BiPO}_{4}$ phases with temperature. 

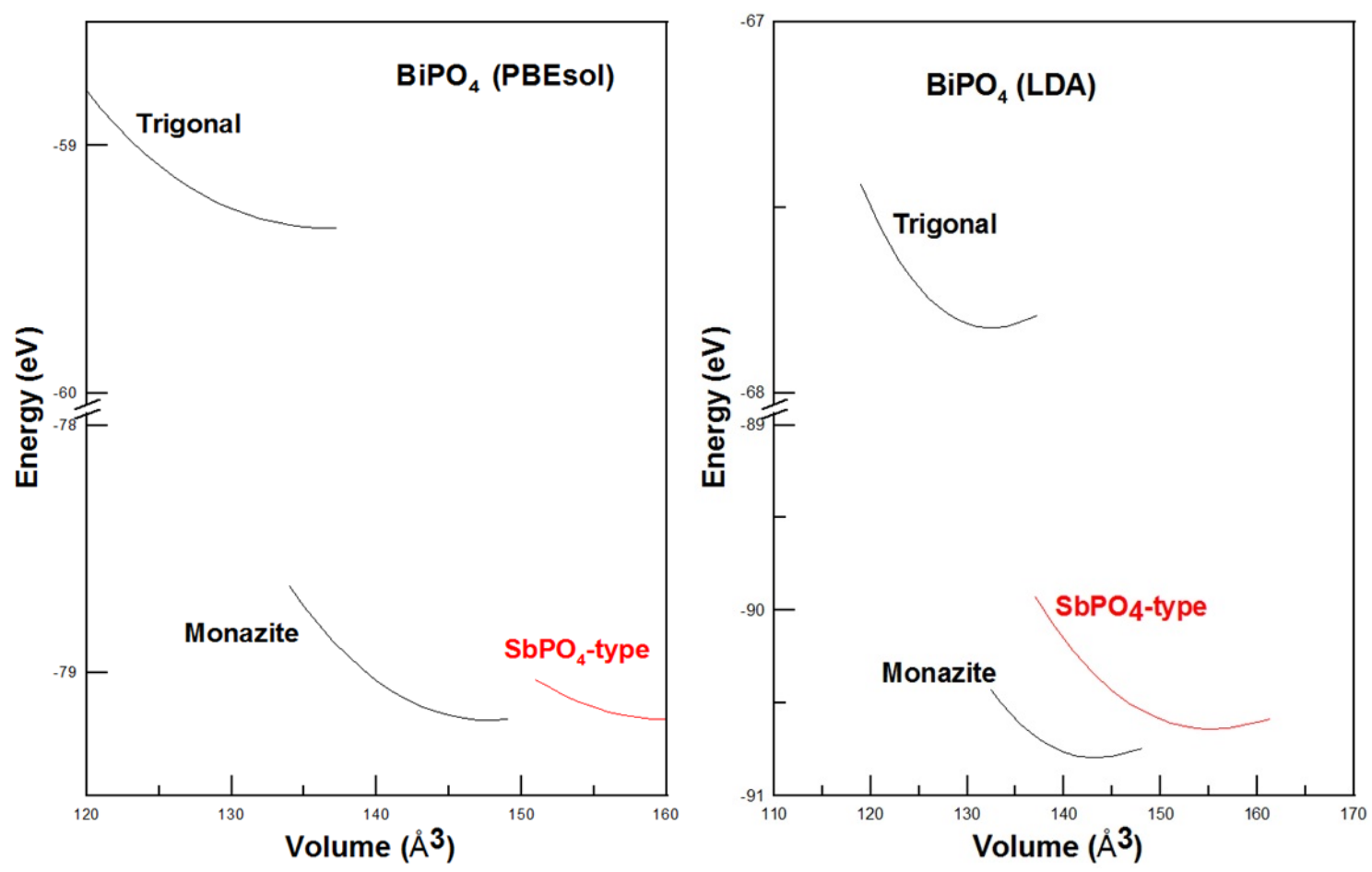

Fig. 8: Total energy as calculated by LDA and PBESol approach. 

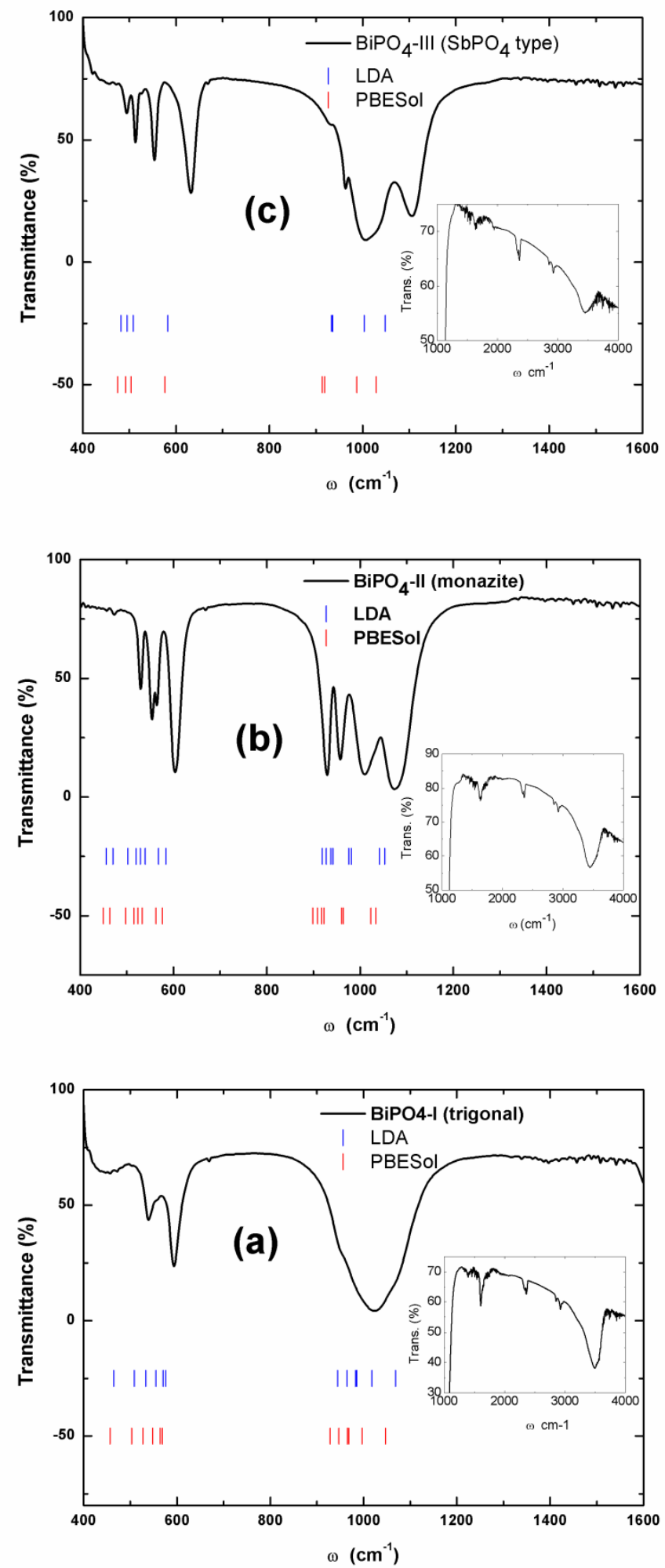

Fig. 9: Typical FTIR spectra of $\mathrm{BiPO}_{4}$ phases. (a). Trigonal (I), (b). Monazite-type (II) and (c). SbPO 4 -type (III). Vertical ticks indicate the calculated frequencies. 

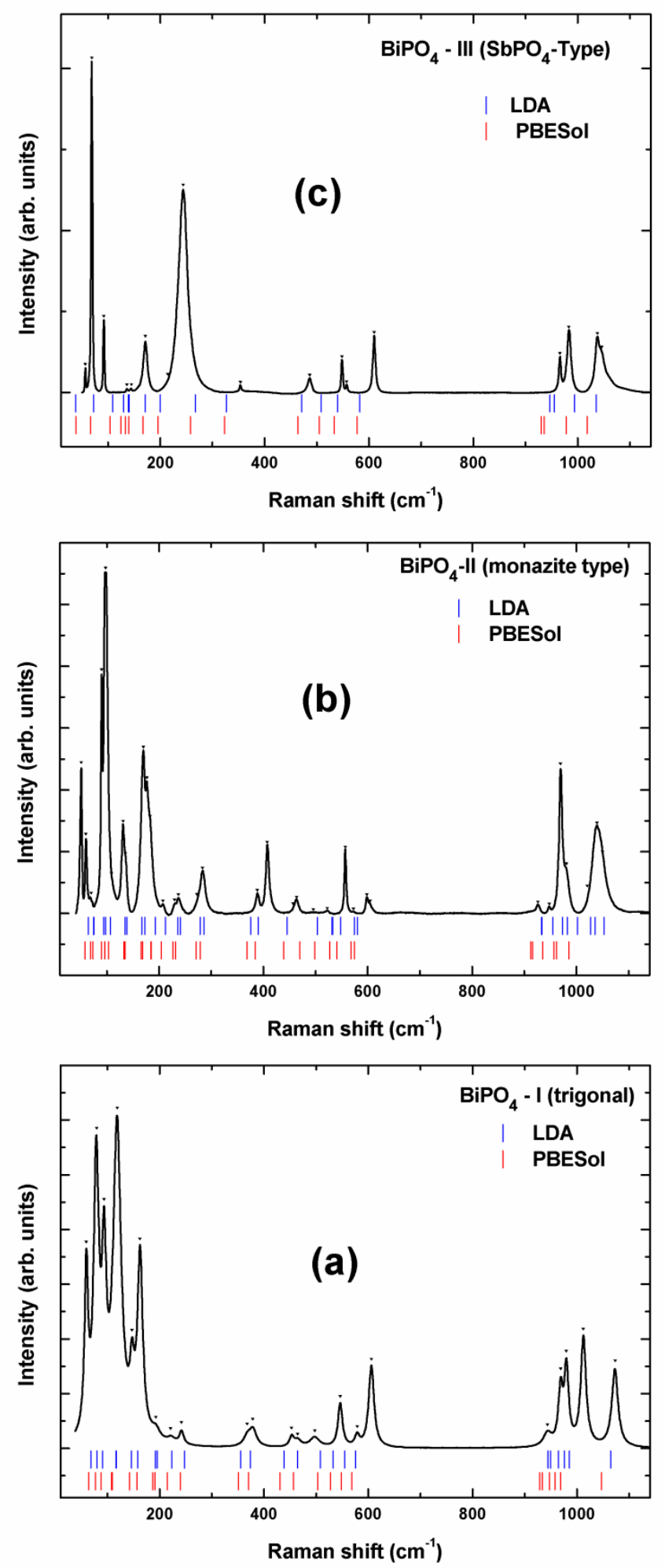

Fig. 10: Typical Raman spectra of $\mathrm{BiPO}_{4}$ phases. (a). Trigonal (I), (b). Monazite-type (II) and (c). SbPO 4 -type (III). Vertical ticks indicate the calculated frequencies. 


\title{
Experimental and theoretical investigations on polymorphism and metastability of $\mathrm{BiPO}_{4}$
}

\author{
S. N. Achary ${ }^{\text {* }}$, D. Errandonea ${ }^{2}$, A. Muñoz ${ }^{3}$, P. Rodríguez-Hernández ${ }^{3}$, F. J. Manjón ${ }^{4}$, \\ P. S. R. Krishna ${ }^{5}$, S. J. Patwe ${ }^{1}$, V. Grover ${ }^{1}$ and A. K. Tyagi ${ }^{1}$ \\ ${ }^{1}$ Chemistry Division, Bhabha Atomic Research Centre, Mumbai 400085, India \\ ${ }^{2}$ MALTA Consolider Team, Departamento de Física Aplicada-ICMUV, Universitad de \\ Valencia, Edificio de Investigación, c/Dr. Moliner 50, Burjassot, 46100 Valencia, Spain \\ ${ }^{3}$ Departamento de Física Fundamental II, Instituto de Materiales y Nanotecnología, MALTA \\ Consolider Team, Universidad de La Laguna, La Laguna 38205, Tenerife, Spain \\ ${ }^{4}$ Instituto de Diseño para la Fabricación y Producción Automatizada, MALTA Consolider \\ Team, Universitat Politècnica de València, 46022 València, Spain \\ ${ }^{5}$ Solid State Physics Division, Bhabha Atomic Research Centre, Mumbai 400085, India
}

\section{Supplementary information}

Table-1. Refined structural parameters of $\mathrm{BiPO}_{4}-\mathrm{I}$ at different temperatures. (from powder XRD data) .
a. Trigonal $\left(\mathrm{P} 3_{1} 21\right)$ phase.
b. Trigonal ( $\left(\mathrm{P}_{1} 21\right)$, Monazite type $\left(\mathrm{P} 2_{1} / \mathrm{n}\right)$ and $\mathrm{SbPO}_{4}$-type $\left(\mathrm{P} 2_{1} / \mathrm{m}\right)$ coexisting phases (at $773 \mathrm{~K}$ ).
c. Monazite type $\left(\mathrm{P} 2_{1} / \mathrm{n}\right)$ and $\mathrm{SbPO}_{4}$-type $\left(\mathrm{P} 2_{1} / \mathrm{m}\right)$ coexisting phases (at $\left.873 \mathrm{~K}\right)$.
d. $\quad \mathrm{SbPO}_{4}$-type $\left(\mathrm{P} 2{ }_{1} / \mathrm{m}\right)$ phase

Table-2. Refined structural parameters of $\mathrm{BiPO}_{4}$-II (Monazite-type) at different temperatures. (from powder XRD data)
a. Monazite type $\left(\mathrm{P}_{1} / \mathrm{n}\right)$ phases.
b. $\mathrm{SbPO}_{4}$-type $\left(\mathrm{P} 2_{1} / \mathrm{m}\right)$ phase.

Table-3. Refined structural parameters of $\mathrm{BiPO}_{4}-\mathrm{III}\left(\mathrm{SbPO}_{4}\right.$ type) at different temperatures. (from powder XRD data) 
d. $\quad \mathrm{SbPO}_{4}$-type $\left(\mathrm{P} 2_{1} / \mathrm{m}\right)$ phase

\begin{tabular}{|c|c|c|c|c|}
\hline \multicolumn{5}{|c|}{ BiPO4-I } \\
\hline Temp & $873 \mathrm{~K}$ & $973 \mathrm{~K}$ & 1073 & 1173 \\
\hline $\mathrm{a}(\AA)$ & $4.8930(5)$ & $4.8964(2)$ & $4.8985(1)$ & $4.8993(2)$ \\
\hline b $(\AA)$ & 7.1088(8) & 7.1154(3) & $7.1238(2)$ & $7.1325(2)$ \\
\hline$c(\AA)$ & $4.7342(5)$ & $4.7421(2)$ & $4.7510(1)$ & $4.7605(2)$ \\
\hline$\beta\left({ }^{\circ}\right)$ & $95.653(9)$ & $95.503(3)$ & $95.341(3)$ & $95.140(3)$ \\
\hline $\mathrm{V}(\AA)^{3}$ & 163.87(3) & $164.46(1)$ & $165.07(1)$ & $165.68(1)$ \\
\hline $\mathrm{Bi} 2 \mathrm{e}$ & $0.1452(9)$ & $0.1505(6)$ & $0.1506(6)$ & $0.1506(7)$ \\
\hline ( x 1/4 z) & 0.25000 & 0.2500 & 0.2500 & 0.2500 \\
\hline & $0.1668(9)$ & $0.1650(6)$ & $0.1659(6)$ & $0.1638(7)$ \\
\hline $\mathrm{P} 2 \mathrm{e}$ & $0.3739(9)$ & $0.3705(9)$ & $0.3686(9)$ & $0.3678(9)$ \\
\hline$(x 3 / 4 z)$ & 0.7500 & 0.7500 & 0.7500 & 0.7500 \\
\hline & $0.3089(10)$ & $0.3106(9)$ & $0.3078(10)$ & $0.3098(10)$ \\
\hline O1 2e & $0.674(1)$ & $0.674(1)$ & $0.671(1)$ & $0.671(1)$ \\
\hline ( x 3/4 z) & 0.750 & 0.7500 & 0.750 & 0.750 \\
\hline & $0.230(2)$ & $0.241(2)$ & $0.234(2)$ & $0.238(2)$ \\
\hline O2 2e & $0.618(2)$ & $0.6114(15)$ & $0.619(2)$ & $0.622(2)$ \\
\hline ( x 1/4 z) & 0.250 & 0.25000 & 0.250 & 0.250 \\
\hline & $0.363(1)$ & $0.3617(10)$ & $0.365(1)$ & $0.364(1)$ \\
\hline O3 4f & $0.203(5)$ & $0.197(3)$ & $0.201(3)$ & $0.204(3)$ \\
\hline$(\mathrm{x} \mathrm{y} \mathrm{z})$ & $0.581(1)$ & $0.591(1)$ & $0.582(2)$ & $0.5824(9)$ \\
\hline & $0.202(6)$ & $0.179(3)$ & $0.194(4)$ & $0.193(4)$ \\
\hline $\mathrm{B}_{\mathrm{ov}}(\AA)^{2}$ & $1.9(2)$ & $1.48(8)$ & $3.63(11)$ & $3.63(12)$ \\
\hline $\mathrm{Rp}, \mathrm{Rwp}, \chi^{2}$ & $15.7,22.0,1.46$ & $18.6,24.8,1.85$ & $18.3,24.6,1.88$ & $18.4,24.8,1.87$ \\
\hline $\mathrm{RB}, \mathrm{RF}$ & $7.73,6.80$ & $8.63,6.41$ & $9.83,8.40$ & $13.4,9.79$ \\
\hline Bi-O1 $(\AA)$ & $2.155(9)$ & $2.183(8)$ & $2.164(8)$ & $2.172(8)$ \\
\hline Bi-O1 & $2.905(9)$ & $2.865(8)$ & $2.904(8)$ & $2.905(8)$ \\
\hline $\mathrm{Bi}-\mathrm{O} 2$ & $2.827(9$ & $2.883(8)$ & $2.852(8)$ & $2.839(8)$ \\
\hline Bi-O2 & $2.408(9)$ & $2.358(7)$ & $2.400(8)$ & $2.418(8)$ \\
\hline Bi-O3 & $2.3731(8)$ & $2.432(7)$ & $2.382(7)$ & $2.388(7)$ \\
\hline $\mathrm{Bi}-\mathrm{O} 3$ & $2.610(22)$ & $2.513(13)$ & $2.602(15)$ & $2.608(15)$ \\
\hline Bi-O3 & $2.610(22)$ & $2.513(12)$ & $2.602(15)$ & $2.608(15)$ \\
\hline Bi-O3 & $2.373(8)$ & $2.432(7)$ & $2.382(7)$ & $2.388(7)$ \\
\hline$<\mathrm{Bi}-\mathrm{O}>_{8}(\AA)$ & 2.532 & 2.522 & 2.536 & 2.541 \\
\hline Distt. & $87.0 \times 10^{-4}$ & $79.7 \times 10^{-4}$ & $87.1 \times 10^{-4}$ & $82.9 \times 10^{-4}$ \\
\hline P-O1 (A) & $1.550(8)$ & $1.552(7)$ & $1.552(7)$ & $1.553(7)$ \\
\hline $\mathrm{P}-\mathrm{O} 2$ & $1.548(7)$ & $1.548(6)$ & $1.550(7)$ & $1.548(7)$ \\
\hline $\mathrm{P}-\mathrm{O} 3$ & $1.522(16)$ & $1.517(11)$ & $1.520(11)$ & $1.517(11)$ \\
\hline $\mathrm{P}-\mathrm{O} 3$ & $1.522(16)$ & $1.517(11)$ & $1.520(11)$ & $1.517(11)$ \\
\hline$<\mathrm{P}-\mathrm{O}>(\AA)$ & 1.536 & 1.534 & 1.536 & 1.534 \\
\hline Distt. & $0.761 \times 10^{-4}$ & $1.13 \times 10^{-4}$ & $1.00 \times 10^{-4}$ & $1.17 \times 10^{-4}$ \\
\hline
\end{tabular}


Table-2. Refined structural parameters of BiPO4-II (Monazite type) at different temperatures. (As observed from powder XRD data)

a. Monazite type

\begin{tabular}{|c|c|c|c|c|c|c|c|c|}
\hline & HT & HT_002 & HT_003 & HT2 & HT2_002 & HT2_003 & HT2_004 & HT2_005 \\
\hline Temp & $298 \mathrm{~K}$ & $373 \mathrm{~K}$ & $473 \mathrm{~K}$ & $573 \mathrm{~K}$ & $673 \mathrm{~K}$ & $773 \mathrm{~K}$ & $873 \mathrm{~K}$ & $973 \mathrm{~K}$ \\
\hline Bi-O1 (A) & $2.568(11)$ & $2.571(11)$ & $2.573(11)$ & $2.555(11)$ & $2.566(11)$ & $2.566(11)$ & $2.610(12)$ & 2.618 \\
\hline Bi-O1 & 2.481(9)) & $2.462(9)$ & $2.458(9)$ & $2.483(10)$ & $2.475(10)$ & $2.484(10)$ & $2.428(11)$ & 2.437 \\
\hline $\mathrm{Bi}-\mathrm{O} 2$ & 3.052(11) & $3.104(11)$ & 3.109(11) & $3.102(12)$ & 3.082(12) & $3.075(12)$ & $3.076(12)$ & 3.088 \\
\hline $\mathrm{Bi}-\mathrm{O} 2$ & 2.396(9) & $2.444(9)$ & $2.428(9)$ & $2.476(9)$ & 2.481(9) & $2.492(9)$ & $2.495(10)$ & 2.504 \\
\hline Bi-O2 & $2.679(10)$ & $2.662(11)$ & $2.663(11)$ & $2.652(11)$ & $2.679(11)$ & $2.706(11)$ & $2.717(12)$ & 2.727 \\
\hline Bi-O3 & 2.314(9) & 2.341(9) & 2.307(9) & $2.330(9)$ & 2.320(9) & $2.350(10)$ & $2.320(10)$ & 2.328 \\
\hline Bi-O3 & $2.507(11)$ & $2.479(11)$ & $2.523(11)$ & $2.540(11)$ & $2.543(11)$ & $2.518(11)$ & $2.566(12)$ & 2.575 \\
\hline Bi-O4 & $2.516(12)$ & $2.574(12)$ & $2.608(12)$ & $2.584(12)$ & $2.665(12)$ & $2.674(12)$ & $2.688(12)$ & 2.699 \\
\hline $\mathrm{Bi}-\mathrm{O} 4$ & $2.445(10)$ & $2.421(10)$ & $2.413(9)$ & $2.422(10)$ & $2.372(9)$ & $2.388(9)$ & $2.390(10)$ & 2.398) \\
\hline$<\mathrm{Bi}-\mathrm{O}>_{9}(\AA)$ & 2.551 & 2.562 & 2.565 & 2.572 & 2.576 & 2.584 & 2.588 & 2.597 \\
\hline Distt. & $62.7 \times 10^{-4}$ & $68.4 \times 10^{-4}$ & $72.5 \times 10^{-4}$ & $65.1 \times 10^{-4}$ & $67.3 \times 10^{-4}$ & $63.4 \times 10^{-4}$ & $68.2 \times 10^{-4}$ & $68.3 \times 10^{-4}$ \\
\hline P-O1 (A) & $1.541(10)$ & $1.536(10)$ & $1.537(10)$ & $1.537(11)$ & $1.537(11)$ & $1.5361(107)$ & $1.539(12)$ & 1.544 \\
\hline P-O2 & $1.556(11)$ & $1.553(11)$ & $1.553(11)$ & $1.551(11)$ & $1.551(11)$ & $1.5520(115)$ & $1.556(12)$ & 1.561 \\
\hline P-O3 & $1.529(8)$ & $1.523(8)$ & $1.528(8)$ & $1.523(8)$ & $1.520(8)$ & $1.5227(84)$ & $1.525(9)$ & 1.530 \\
\hline P-O4 & 1.497(12) & $1.494(12)$ & $1.498(12)$ & $1.493(12)$ & $1.494(12)$ & $1.4964(112)$ & $1.496(12)$ & 1.501 \\
\hline$<\mathrm{P}-\mathrm{O}>_{4}(\AA)$ & 1.531 & 1.527 & 1.529 & 1.526 & 1.525 & 1.527 & 1.529 & 1.534 \\
\hline Distt. & $1.98 \times 10^{-4}$ & $2.00 \times 10^{-4}$ & $1.73 \times 10^{-4}$ & $1.94 \times 10^{-4}$ & $1.93 \times 10^{-4}$ & $1.78 \times 10^{-4}$ & $2.03 \times 10^{-4}$ & $2.06 \times 10^{-4}$ \\
\hline
\end{tabular}




\section{b. $\quad \mathrm{SbPO}_{4}$ - type}

\begin{tabular}{|c|c|c|c|c|}
\hline & & HT2_005 (R) & HT2_006 (R) & HT2_007 (R) e \\
\hline Temp & & $973 \mathrm{~K}$ & $1073 \mathrm{~K}$ & $1173 \mathrm{~K}$ \\
\hline a $(\AA)$ & & 4.8991(1) & $4.9004(2)$ & $4.9022(2)$ \\
\hline b $(\AA)$ & & 7.1173(2) & 7.1257(2) & $7.1344(3)$ \\
\hline с $(\AA)$ & & $4.7441(1)$ & $4.7523(2)$ & 4.7621(3) \\
\hline$\beta\left(^{\circ}\right)$ & & $95.514(2)$ & $95.358(3)$ & $95.171(5)$ \\
\hline $\mathrm{V}(\AA)^{3}$ & & $164.653(8)$ & $165.219(9)$ & $165.876(14)$ \\
\hline $\mathrm{Bi}$ & $2 \mathrm{e}$ & $0.1477(6)$ & $0.1499(6)$ & $0.1463(9)$ \\
\hline & & 0.2500 & 0.2500 & 0.2500 \\
\hline & & $0.1638(6)$ & $0.1650(6)$ & $0.1638(9)$ \\
\hline & & & & 1.9141 \\
\hline $\mathrm{P}$ & $2 \mathrm{e}$ & $0.3680(10)$ & $0.3691(9)$ & $0.3603(9)$ \\
\hline & & 0.7500 & 0.7500 & 0.7500 \\
\hline & & $0.3139(9)$ & $0.3094(10)$ & $0.3117(10)$ \\
\hline & & & & 0.2912 \\
\hline $\mathrm{O} 1$ & $2 \mathrm{e}$ & $0.669(1)$ & $0.671(1)$ & $0.663(1)$ \\
\hline & & 0.750 & 0.750 & 0.750 \\
\hline & & $0.240(2)$ & $0.237(2)$ & $0.237(2)$ \\
\hline & & & & 4.3468 \\
\hline $\mathrm{O} 2$ & $2 \mathrm{e}$ & $0.615(2)$ & $0.618(2)$ & $0.619(2)$ \\
\hline & & 0.250 & 0.250 & 0.250 \\
\hline & & $0.360(1)$ & $0.364(1)$ & $0.361(1)$ \\
\hline & & & & 4.3468 \\
\hline $\mathrm{O} 3$ & $4 \mathrm{f}$ & $0.229(3)$ & $0.208(3)$ & $0.193(5)$ \\
\hline & & $0.579(1)$ & $0.583(1)$ & $0.581(1)$ \\
\hline & & $0.182(4)$ & $0.188(4)$ & $0.205(6)$ \\
\hline & & & & 4.3468 \\
\hline Bov & & $-0.57(8)$ & $1.7(1)$ & \\
\hline Rp, Rwp, $\chi^{2}$ & & $20.3,25.8,2.69$ & $22.3,27.1,2.93$ & $28.2,34.8,4.36$ \\
\hline $\mathrm{RB}, \mathrm{RF}$ & & $20.9,10.3$ & $19.9,11.3$ & $30.8,18.6$ \\
\hline Bi - O1 $(\AA)$ & & $2.193(8)$ & $2.175(8)$ & $2.200(9)$ \\
\hline $\mathrm{Bi}-\mathrm{O} 1$ & & $2.884(8)$ & $2.895(8)$ & 2.922(9) \\
\hline $\mathrm{Bi}-\mathrm{O} 2$ & & $2.853(8)$ & $2.856(8)$ & 2.829(9) \\
\hline $\mathrm{Bi}-\mathrm{O} 2$ & & $2.389(8)$ & $2.396(8)$ & 2.421(9) \\
\hline $\mathrm{Bi}-\mathrm{O} 3$ & & 2.373(6) & $2.388(7)$ & $2.380(8)$ \\
\hline $\mathrm{Bi}-\mathrm{O} 3$ & & $2.646(14)$ & $2.601(14)$ & $2.603(23)$ \\
\hline $\mathrm{Bi}-\mathrm{O} 3$ & & $2.646(14)$ & $2.601(15)$ & $2.603(23)$ \\
\hline $\mathrm{Bi}-\mathrm{O} 3$ & & $2.373(6)$ & $2.388(7)$ & $2.380(8)$ \\
\hline$<\mathrm{Bi}-\mathrm{O}>(\AA)$ & & 2.545 & 2.537 & 2.542 \\
\hline Distt. & & $84.5 \times 10^{-4} \backslash$ & $84.2 \times 10^{-4}$ & $81.0 \times 10^{-4}$ \\
\hline $\mathrm{P}-\mathrm{O} 1(\AA)$ & & $1.547(8)$ & $1.5513(73)$ & $1.5565(77)$ \\
\hline $\mathrm{P}-\mathrm{O} 2$ & & $1.540(6)$ & $1.5474(67)$ & $1.5518(67)$ \\
\hline P - O3 & & $1.504(11)$ & $1.5151(111)$ & $1.5191(162)$ \\
\hline $\mathrm{P}-\mathrm{O} 3$ & & $1.504(11)$ & $1.5151(111)$ & $1.5191(162)$ \\
\hline$<\mathrm{P}-\mathrm{O}>(\AA)$ & & 1.524 & 1.532 & 1.537 \\
\hline Distt. & & $1.70 \times 10^{-4}$ & $1.26 \times 10^{-4}$ & $1.31 \times 10^{-4}$ \\
\hline
\end{tabular}




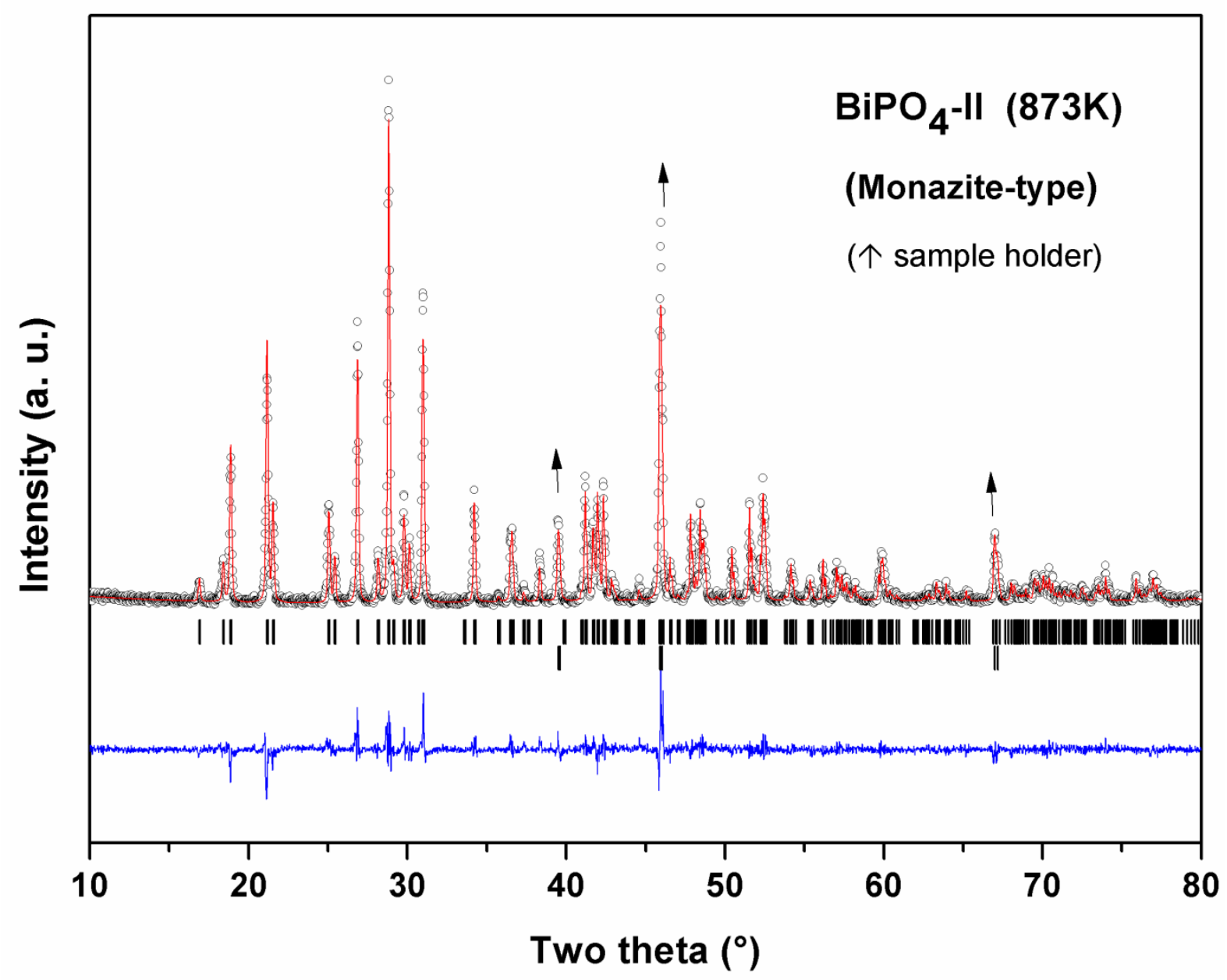

Fig.4. Rietveld refinement plot of the powder XRD data of $\mathrm{BiPO}_{4}-\mathrm{II}$ at $873 \mathrm{~K}$. (only monazite -type phase is observed). Arrows indicate reflections due to sample holder). 


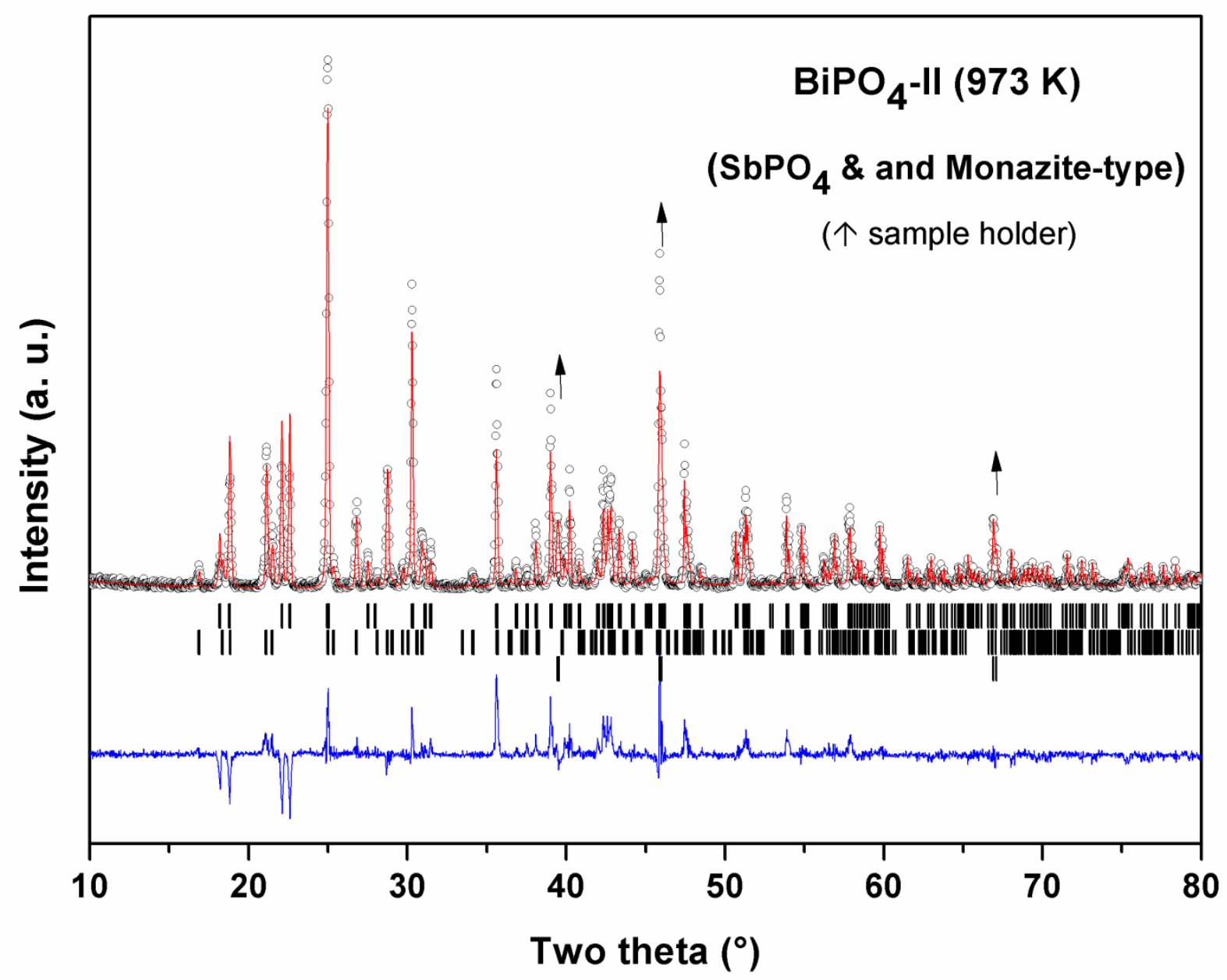

Fig.5. Rietveld refinement plot of the powder XRD data of $\mathrm{BiPO}_{4}-\mathrm{II}$ at $973 \mathrm{~K}$. (coexistence of monazite and $\mathrm{SbPO}_{4}$-type phases are indicated by vertical ticks (from top to bottom). Arrows indicate reflections due to sample holder). 


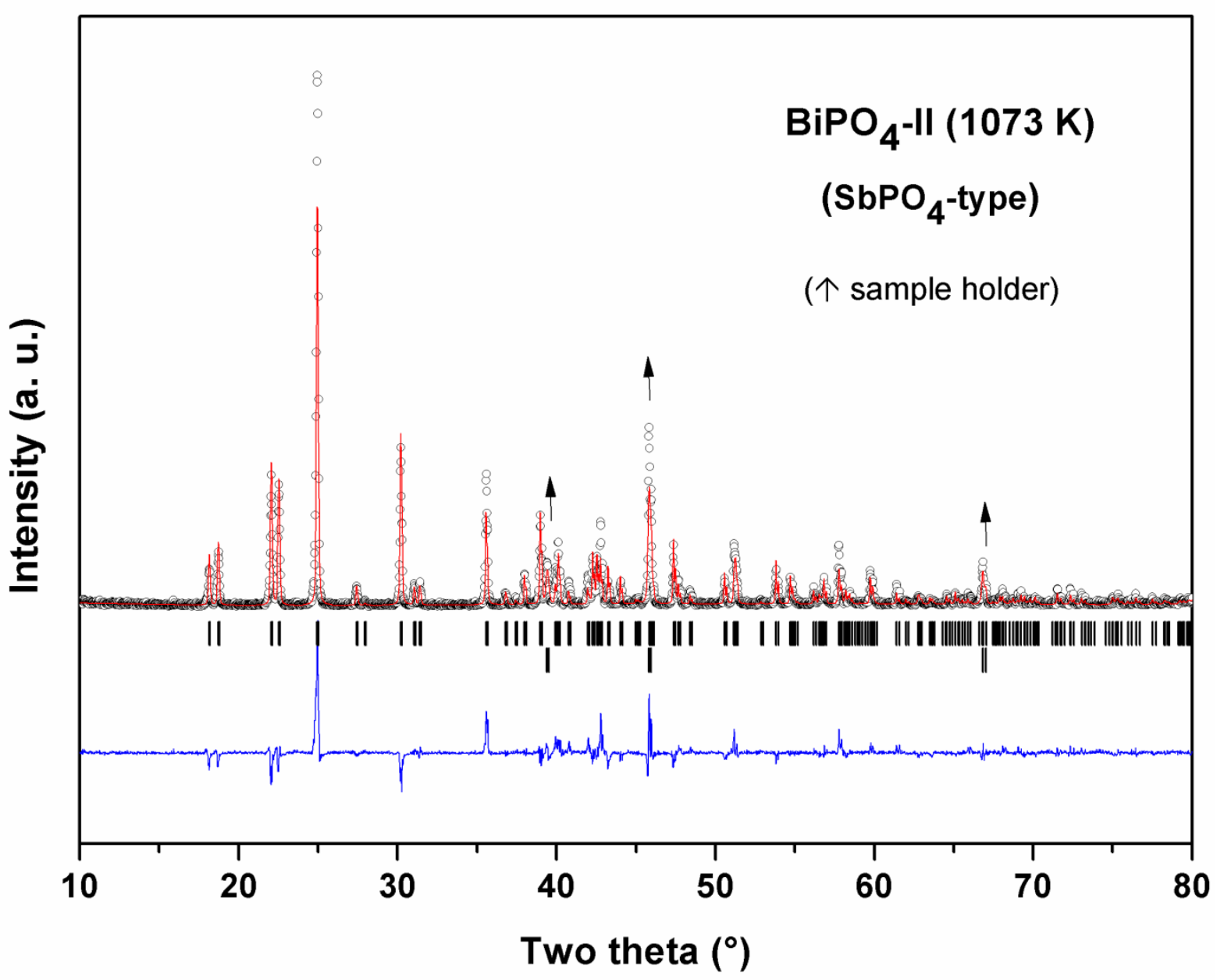

Fig.6. Rietveld refinement plot of the powder XRD data of $\mathrm{BiPO}_{4}$-II at $1073 \mathrm{~K}$. (Only $\mathrm{SbPO}_{4}$ type phase is observed). Arrows indicate reflections due to sample holder) 
Table-3. Refined structural parameters of BiPO4-III ( $\mathrm{SbPO}_{4}$ type) at different temperatures. (As observed from powder XRD data)

\begin{tabular}{|c|c|c|c|c|c|c|c|c|c|c|}
\hline & HT & 002 & 003 & 004 & 005 & 006 & 007 & 008 & 009 & 010 \\
\hline Temp & 298 & 373 & 473 & 573 & 673 & 773 & 873 & 973 & 1073 & 1173 \\
\hline a $(\AA)$ & $4.8804(1)$ & $4.8828(1)$ & $4.8857(1)$ & $4.8884(1)$ & $4.8912(1)$ & $4.8936(1)$ & $4.8959(1)$ & $4.8980(1)$ & $4.8997(1)$ & $4.9015(1)$ \\
\hline $\mathrm{b}(\AA)$ & 7.0684(2) & $7.0729(2)$ & $7.0797(2)$ & 7.0867(2) & $7.0935(2)$ & $7.1011(2)$ & 7.10897(18) & 7.1169(2) & $7.1252(2)$ & $7.1346(2)$ \\
\hline c $(\AA)$ & $4.7033(1)$ & $4.7067(1)$ & $4.7116(1)$ & $4.7170(1)$ & $4.7228(1)$ & $4.7290(1)$ & $4.73601(12)$ & $4.7434(1)$ & $4.7520(1)$ & $4.7618(1)$ \\
\hline$\beta\left(^{\circ}\right)$ & $96.285(3)$ & $96.219(3)$ & $96.126(2)$ & $96.022(2)$ & $95.915(2)$ & $95.792(2)$ & $95.662(2)$ & $95.510(2)$ & $95.342(2)$ & $95.146(2)$ \\
\hline $\mathrm{V}(\AA)^{3}$ & $161.272(8)$ & 161.591(8) & $162.039(7)$ & $162.508(7)$ & $162.985(7)$ & $163.496(7)$ & $164.032(7)$ & $164.583(7)$ & $165.177(7)$ & $165.849(7)$ \\
\hline \multirow{4}{*}{$\begin{array}{c}\text { BI } \\
\text { x y z Biso } \AA^{2}\end{array}$} & $0.1433(4)$ & $0.1437(4)$ & $0.1444(4)$ & $0.1462(4)$ & $0.1458(4)$ & $0.1477(4)$ & $0.1484(5)$ & $0.1491(5)$ & $0.1495(5)$ & $0.1496(6)$ \\
\hline & 0.2500 & 0.2500 & 0.2500 & 0.2500 & 0.2500 & 0.2500 & 0.2500 & 0.2500 & 0.2500 & 0.2500 \\
\hline & $0.1681(4)$ & $0.1673(4)$ & $0.1664(4)$ & $0.1671(4)$ & $0.1662(4)$ & $0.1658(4)$ & $0.1653(4)$ & $0.1652(5)$ & $0.1645(5)$ & $0.1648(6)$ \\
\hline & $0.33(5)$ & $0.21(5)$ & $0.28(5)$ & $0.34(5)$ & $0.57(6)$ & $0.72(6)$ & $0.93(6)$ & $1.25(6)$ & $1.60(7)$ & $1.91(8)$ \\
\hline \multirow[t]{4}{*}{$\mathrm{P}$} & $0.3751(9)$ & $0.3741(9)$ & $0.3724(9)$ & $0.3697(9)$ & $0.3717(9)$ & $0.3699(9)$ & $0.3696(9)$ & $0.3691(9)$ & $0.3689(9)$ & $0.3683(9)$ \\
\hline & 0.7500 & 0.7500 & 0.7500 & 0.7500 & 0.7500 & 0.7500 & 0.7500 & 0.7500 & 0.7500 & 0.7500 \\
\hline & $0.3092(9)$ & $0.3067(9)$ & $0.3065(9)$ & $0.3088(9)$ & $0.3090(9)$ & $0.3094(9)$ & $0.3094(9)$ & $0.3081(9)$ & $0.3082(9)$ & $0.3086(10)$ \\
\hline & 0.32 & 0.32 & 0.32 & 0.32 & 0.32 & 0.32 & 0.320 & 0.32 & $0.2(3)$ & $0.3(4)$ \\
\hline \multirow[t]{4}{*}{ O1 } & $0.676(2)$ & $0.673(2)$ & $0.674(2)$ & $0.672(2)$ & $0.672(2)$ & $0.671(2)$ & $0.671(2)$ & $0.672(2)$ & $0.670(2)$ & $0.670(2)$ \\
\hline & 0.750 & 0.7500 & 0.750 & 0.750 & 0.7500 & 0.750 & 0.750 & 0.750 & 0.750 & 0.750 \\
\hline & $0.234(4)$ & $0.225(4)$ & $0.232(4)$ & $0.238(4)$ & $0.231(4)$ & $0.235(5)$ & $0.236(5)$ & $0.238(5)$ & $0.234(5)$ & $0.235(6)$ \\
\hline & $1.0(3)$ & $0.7(3)$ & $0.8(3)$ & $1.0(3)$ & $1.0(3)$ & $1.6(4)$ & $2.2(4)$ & $2.0(4)$ & $3.1(4)$ & $4.3(5)$ \\
\hline \multirow[t]{4}{*}{$\mathrm{O} 2$} & $0.603(4)$ & $0.601(4)$ & $0.608(4)$ & $0.611(4)$ & $0.611(4)$ & $0.609(5)$ & $0.611(5)$ & $0.617(5)$ & $0.619(5)$ & $0.622(7)$ \\
\hline & 0.250 & 0.2500 & 0.2500 & 0.2500 & 0.2500 & 0.250 & 0.250 & 0.250 & 0.250 & 0.250 \\
\hline & $0.361(1)$ & $0.364(1)$ & $0.365(1)$ & $0.363(1)$ & $0.363(1)$ & 0.363(1 & $0.363(1)$ & $0.365(1)$ & $0.366(1)$ & $0.365(1)$ \\
\hline & $1.0(3)$ & $0.7(3)$ & $0.8(3)$ & $1.0(3)$ & $1.0(3)$ & $1.6(4)$ & $2.2(4)$ & $2.0(4)$ & $3.1(4)$ & $4.3(5)$ \\
\hline \multirow[t]{4}{*}{ O3 } & $0.220(3)$ & $0.220(3)$ & $0.218(3)$ & $0.217(3)$ & $0.218(3)$ & $0.212(3)$ & $0.211(3)$ & $0.212(3)$ & $0.204(3)$ & $0.202(4)$ \\
\hline & $0.578(1)$ & $0.577(1)$ & $0.577(1)$ & $0.577(1)$ & $0.577(1)$ & $0.580(1)$ & $0.578(1)$ & $0.578(1)$ & $0.581(2)$ & $0.581(2)$ \\
\hline & $0.183(3)$ & $0.184(3)$ & $0.188(3)$ & $0.188(3)$ & $0.191(3)$ & $0.186(3)$ & $0.193(3)$ & $0.194(3)$ & $0.195(4)$ & $0.199(4)$ \\
\hline & $1.0(3)$ & $0.7(3)$ & $0.8(3)$ & $1.0(3)$ & $1.0(3)$ & $1.6(4)$ & $2.2(4)$ & $2.0(4)$ & $3.1(4)$ & $4.3(5)$ \\
\hline $\begin{array}{c}\text { Rp, Rwp } \\
\chi^{2}\end{array}$ & $\begin{array}{l}13.5,19.0, \\
1.60\end{array}$ & $\begin{array}{l}\text { 13.4, 18.6, } \\
1.54\end{array}$ & $\begin{array}{l}\text { 13.2, 18.7, } \\
1.56\end{array}$ & $\begin{array}{l}\text { 13.0,18.3, } \\
1.51\end{array}$ & $\begin{array}{l}\text { 13.6, 18.7, } \\
1.58\end{array}$ & $\begin{array}{l}\text { 13.4, 18.8, } \\
1.60\end{array}$ & $\begin{array}{l}13.5,19.0, \\
1.63\end{array}$ & $\begin{array}{l}\text { 13.6, 18.8, } \\
1.61\end{array}$ & $\begin{array}{l}\text { 14.4, 19.6, } \\
1.74\end{array}$ & $\begin{array}{l}15.7,21.2, \\
1.90\end{array}$ \\
\hline $\mathrm{RB}, \mathrm{RF}$ & $5.97,3.41$ & $5.62,3.35$ & $5.98,3.53$ & $5.41,3.52$ & $5.48,3.87$ & $5.65,3.90$ & $5.43,4.32$ & $5.98,4.90$ & $6.52,5.30$ & $7.17,6.06$ \\
\hline
\end{tabular}


Table-3. Refined structural parameters of $\mathrm{BiPO}_{4}$-III ( $\mathrm{SbPO}_{4}$ type) at different temperatures. (As observed from powder XRD data)

\begin{tabular}{|c|c|c|c|c|c|c|c|c|c|c|}
\hline & HT & 002 & 003 & 004 & 005 & 006 & 007 & 008 & 009 & 010 \\
\hline $\begin{array}{ll}\mathrm{Bi}-\mathrm{O} 1 & (\AA) \\
\mathrm{Bi}-\mathrm{I} 1 & \\
\mathrm{Bi}-\mathrm{O} 2 & \\
\mathrm{Bi}-\mathrm{O} 2 & \\
\mathrm{Bi}-\mathrm{O} 3 & \\
\mathrm{Bi}-\mathrm{O} 3 & \\
\mathrm{Bi}-\mathrm{O} 3 & \\
\mathrm{Bi}-\mathrm{O} 3 & \end{array}$ & \begin{tabular}{|l|}
$2.172(18)$ \\
$2.853(18)$ \\
$2.881(18)$ \\
$2.326(18)$ \\
$2.351(9)$ \\
$2.589(13)$ \\
$2.589(13)$ \\
$2.351(9)$ \\
\end{tabular} & $\begin{array}{l}2.138(18) \\
2.903(18) \\
2.899(19) \\
2.322(18) \\
2.343(9) \\
2.598(13) \\
2.598(13) \\
2.343(9)\end{array}$ & $\begin{array}{l}2.161(18) \\
2.879(18) \\
2.874(19) \\
2.356(18) \\
2.343(9) \\
2.606(13) \\
2.606(13) \\
2.343(9)\end{array}$ & $\begin{array}{l}2.191(18) \\
2.853(18) \\
2.864(19) \\
2.361(18) \\
2.341(9) \\
2.616(13) \\
2.616(13) \\
2.341(9)\end{array}$ & $\begin{array}{l}2.160(18) \\
2.895(18) \\
2.864(19) \\
2.367(18) \\
2.345(9) \\
2.628(13) \\
2.628(13) \\
2.345(9)\end{array}$ & $\begin{array}{l}2.172(22) \\
2.883(23) \\
2.883(23) \\
2.353(22) \\
2.364(10) \\
2.595(13) \\
2.595(13) \\
2.364(10)\end{array}$ & $\begin{array}{l}2.173(22) \\
2.885(23) \\
2.878(23) \\
2.364(23) \\
2.355(10) \\
2.623(13) \\
2.623(13) \\
2.355(10)\end{array}$ & $\begin{array}{l}2.180(22) \\
2.882(23) \\
2.857(23) \\
2.394(23) \\
2.358(10) \\
2.636(13) \\
2.636(13) \\
2.358(10)\end{array}$ & \begin{tabular}{|l|}
$2.164(22)$ \\
$2.912(23)$ \\
$2.852(23)$ \\
$2.407(23)$ \\
$2.378(12)$ \\
$2.610(15)$ \\
$2.610(15)$ \\
$2.378(12))$
\end{tabular} & \begin{tabular}{|l|}
$2.170(27)$ \\
$2.915(27)$ \\
$2.837(32)$ \\
$2.424(32)$ \\
$2.382(14)$ \\
$2.623(18)$ \\
$2.623(18)$ \\
$2.382(15)$
\end{tabular} \\
\hline$<\mathrm{P}-\mathrm{O}>(\AA)$ & 2.514 & 2.518 & 2.521 & 2.523 & 2.529 & 2.526 & 2.532 & 2.538 & \begin{tabular}{|l}
2.539 \\
\end{tabular} & \begin{tabular}{|l}
2.544 \\
\end{tabular} \\
\hline $\begin{array}{ll}\text { Distt. } & \\
\text { P-O1 } & (\AA) \\
\text { P-O2 } & \\
\text { P-O3 } & \\
\text { P-O3 } & \end{array}$ & \begin{tabular}{|l|}
$92.3 \times 10^{-4}$ \\
$1.549(11)$ \\
$1.543(7)$ \\
$1.516(11)$ \\
$1.516(11)$ \\
\end{tabular} & $\begin{array}{l}108.4 \times 10^{-4} \\
1.548(12) \\
1.544(6) \\
1.517(11) \\
1.517(11)\end{array}$ & $\begin{array}{l}95.8 \times 10^{-4} \\
1.548(11) \\
1.542(7) \\
1.514(11) \\
1.514(11)\end{array}$ & $\begin{array}{l}87.5 \times 10^{-4} \\
1.548(11) \\
1.543(7) \\
1.518(11) \\
1.518(11)\end{array}$ & $\begin{array}{l}97.0 \times 10^{-4} \\
1.548(11) \\
1.544(7) \\
1.517(11) \\
1.517(11)\end{array}$ & $\begin{array}{l}92.4 \times 10^{-4} \\
1.549(12) \\
1.544(7) \\
1.519(12) \\
1.519(12)\end{array}$ & $\begin{array}{l}93.8 \times 10^{-4} \\
1.551(12) \\
1.545(7) \\
1.521(12) \\
1.521(12)\end{array}$ & $\begin{array}{l}88.0 \times 10^{-4} \\
1.550(12) \\
1.547(7) \\
1.517(12) \\
1.517(12)\end{array}$ & $\begin{array}{l}88.7 \times 10^{-4} \\
1.548(13) \\
1.546(7) \\
1.519(13) \\
1.519(13)\end{array}$ & $\begin{array}{l}85.3 \times 10^{-4} \\
1.550(14) \\
1.549(7) \\
1.519(16) \\
1.519(16)\end{array}$ \\
\hline
\end{tabular}




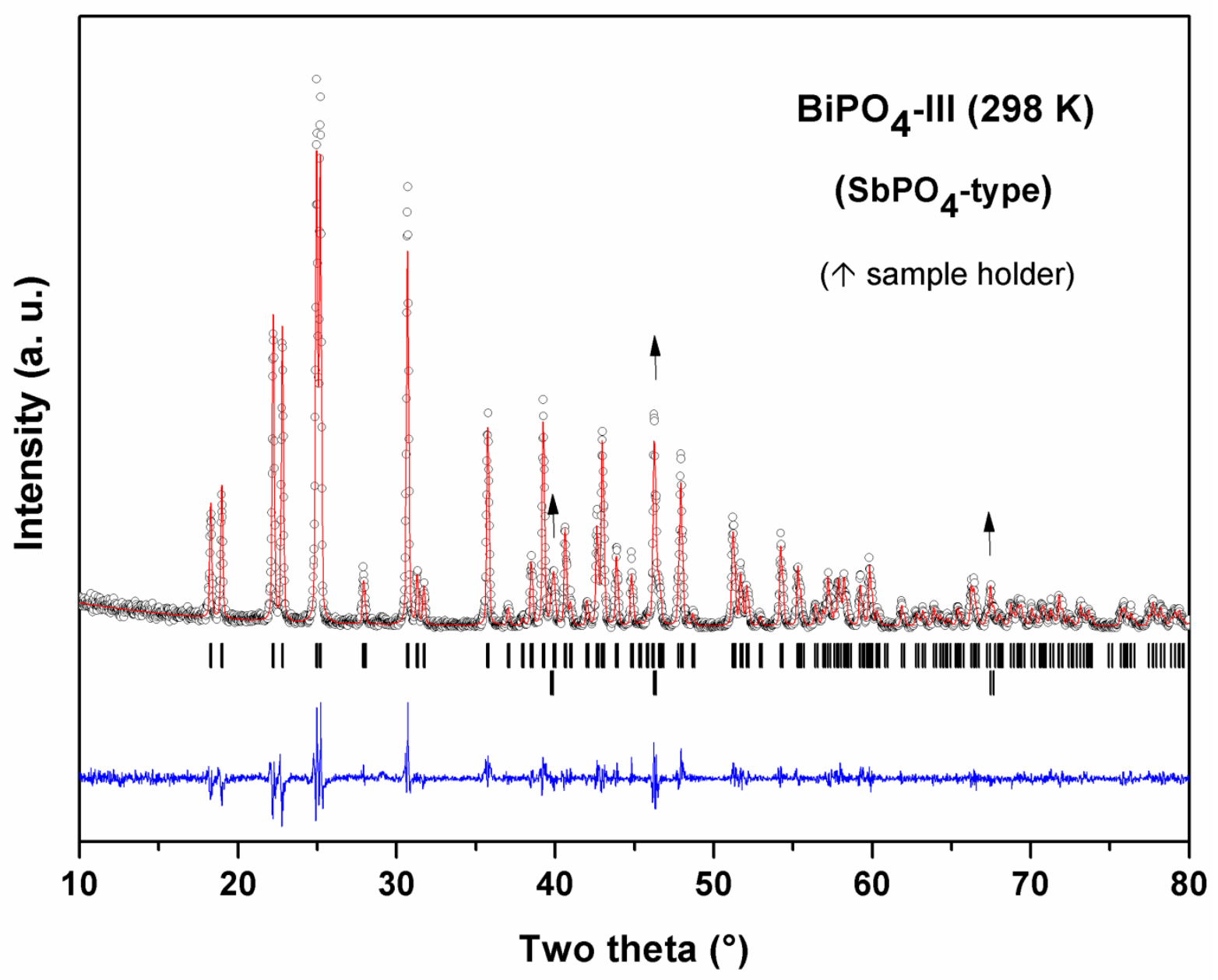

Fig.7. Rietveld refinement plot of the powder XRD data of $\mathrm{BiPO}_{4}-\mathrm{III}$ at $298 \mathrm{~K}$. (Only $\mathrm{SbPO}_{4^{-}}$ type phases is observed). Arrows indicate reflections due to sample holder) 


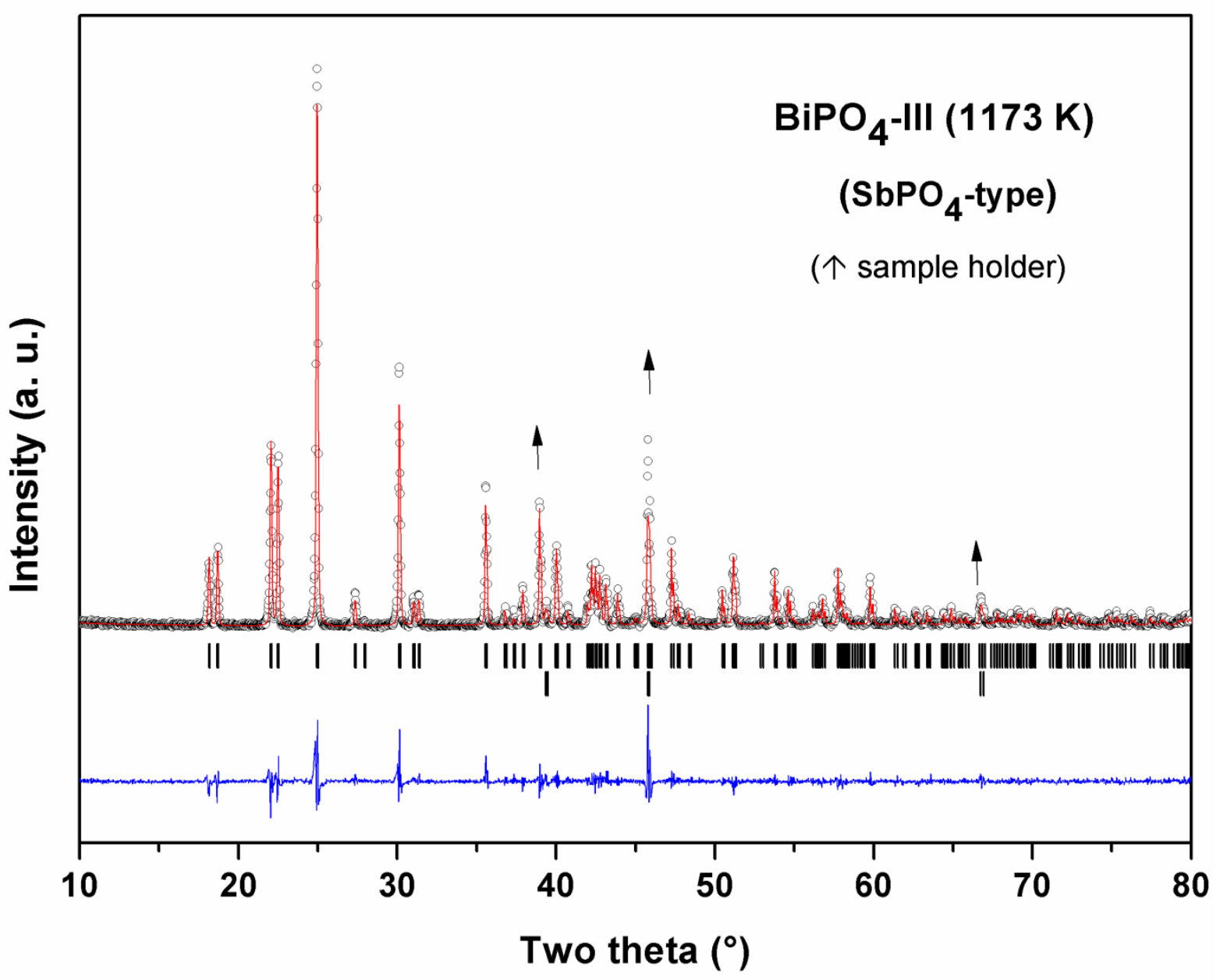

Fig.8. Rietveld refinement plot of the powder XRD data of $\mathrm{BiPO}_{4}$-III at $1173 \mathrm{~K}$. (Only $\mathrm{SbPO}_{4}$-type phase is observed). Arrows indicate reflections due to sample holder) 


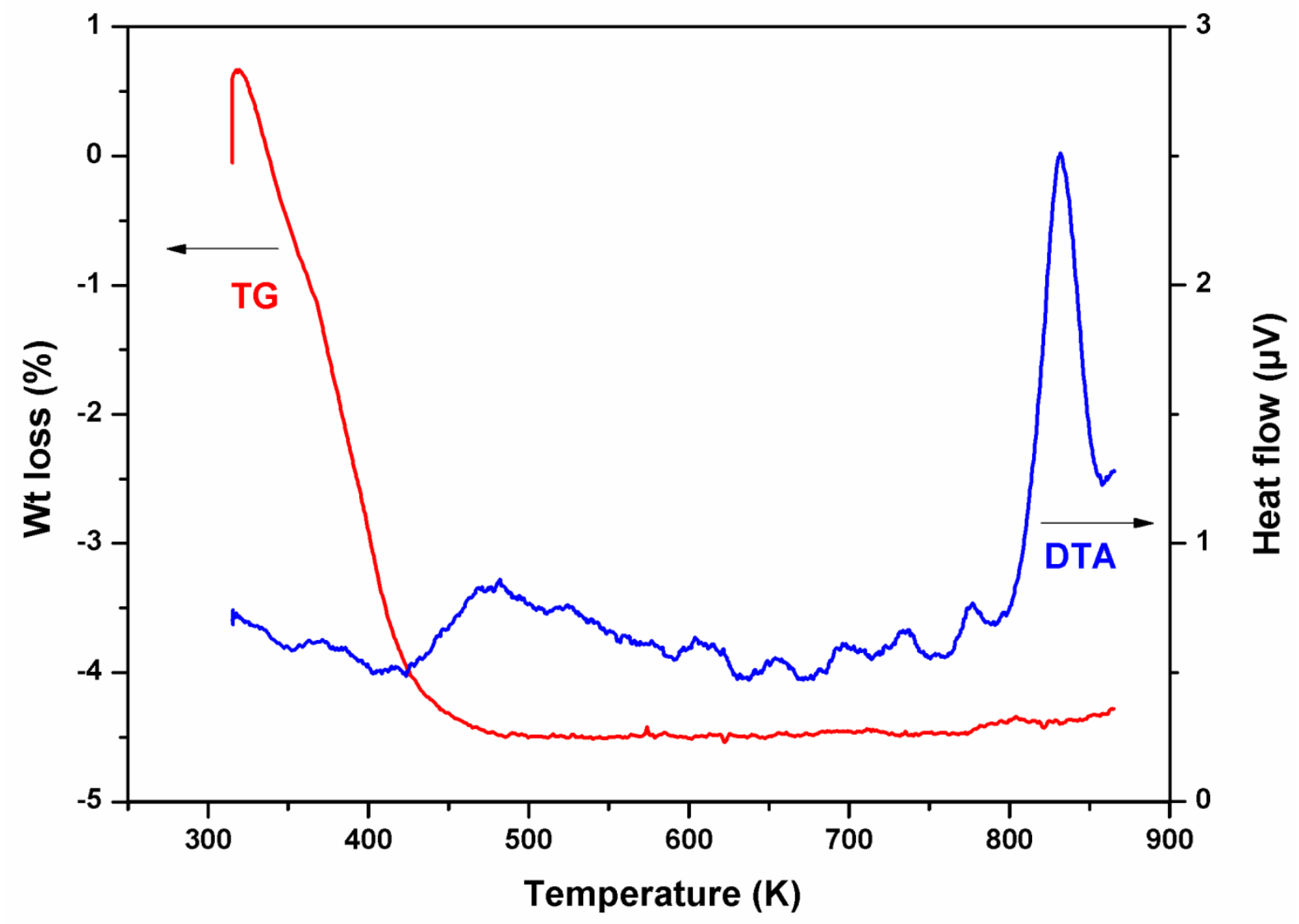

Fig.9. Typical TG-DTA traces of as prepared sample of hydrated trigonal $\mathrm{BiPO}_{4}$ sample. (Sample was heated from 323 to $873 \mathrm{~K}$ at a rate of $10 \mathrm{~K} / \mathrm{min}$ ). 


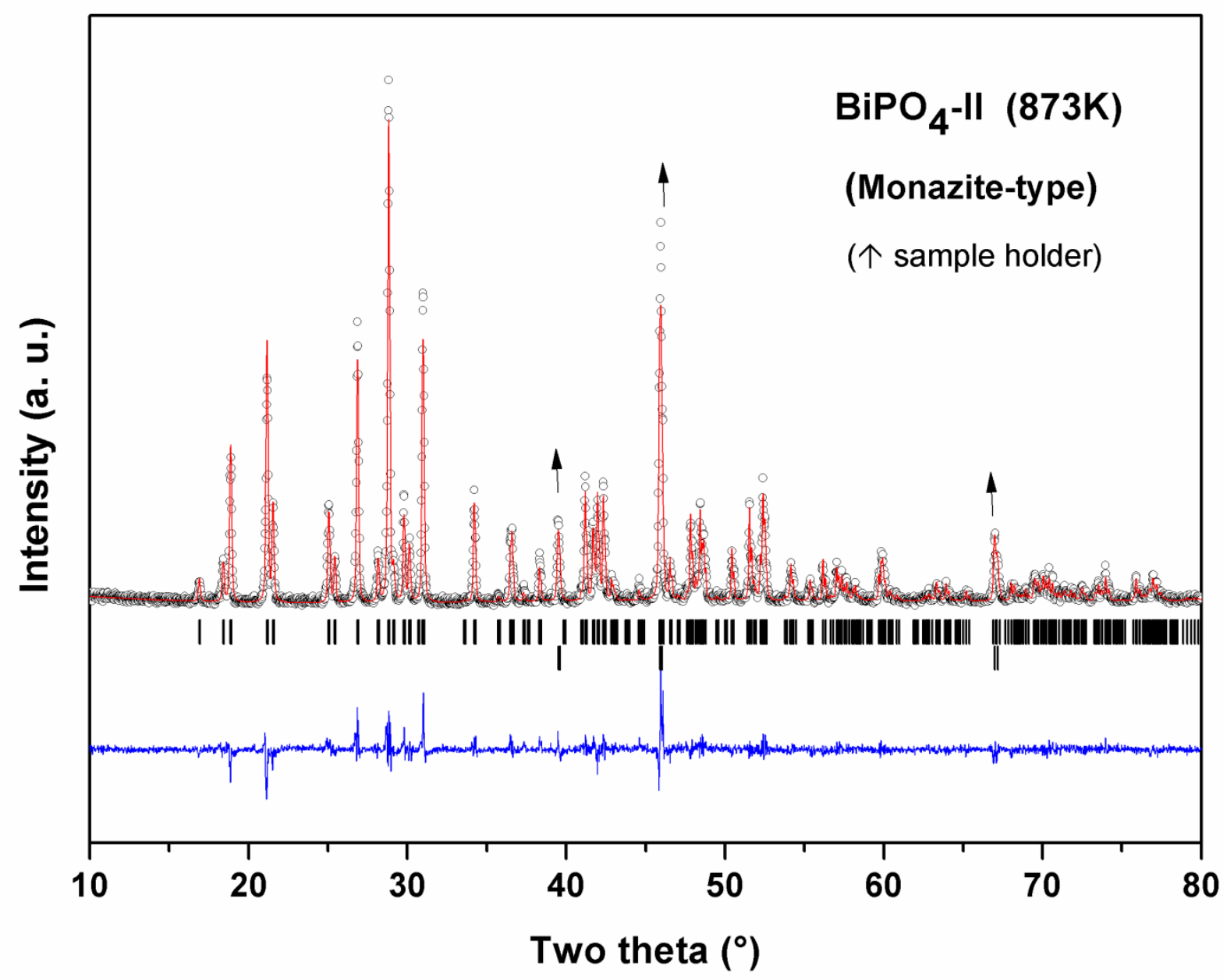

Fig.4. Rietveld refinement plot of the powder XRD data of $\mathrm{BiPO}_{4}-\mathrm{II}$ at $873 \mathrm{~K}$. (only monazite -type phase is observed). Arrows indicate reflections due to sample holder). 


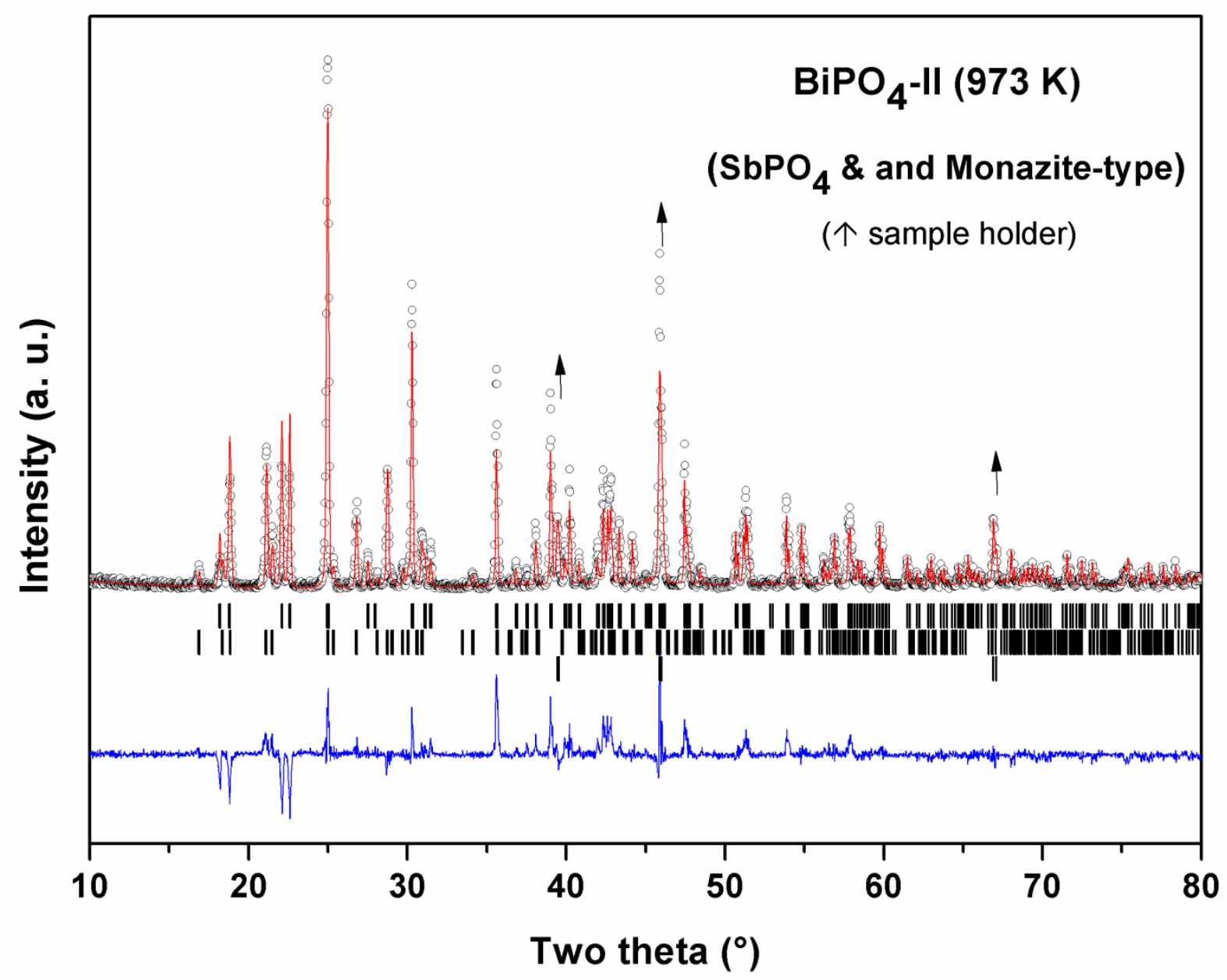

Fig.5. Rietveld refinement plot of the powder XRD data of $\mathrm{BiPO}_{4}-\mathrm{II}$ at $973 \mathrm{~K}$. (coexistence of monazite and $\mathrm{SbPO}_{4}$-type phases are indicated by vertical ticks (from top to bottom). Arrows indicate reflections due to sample holder). 


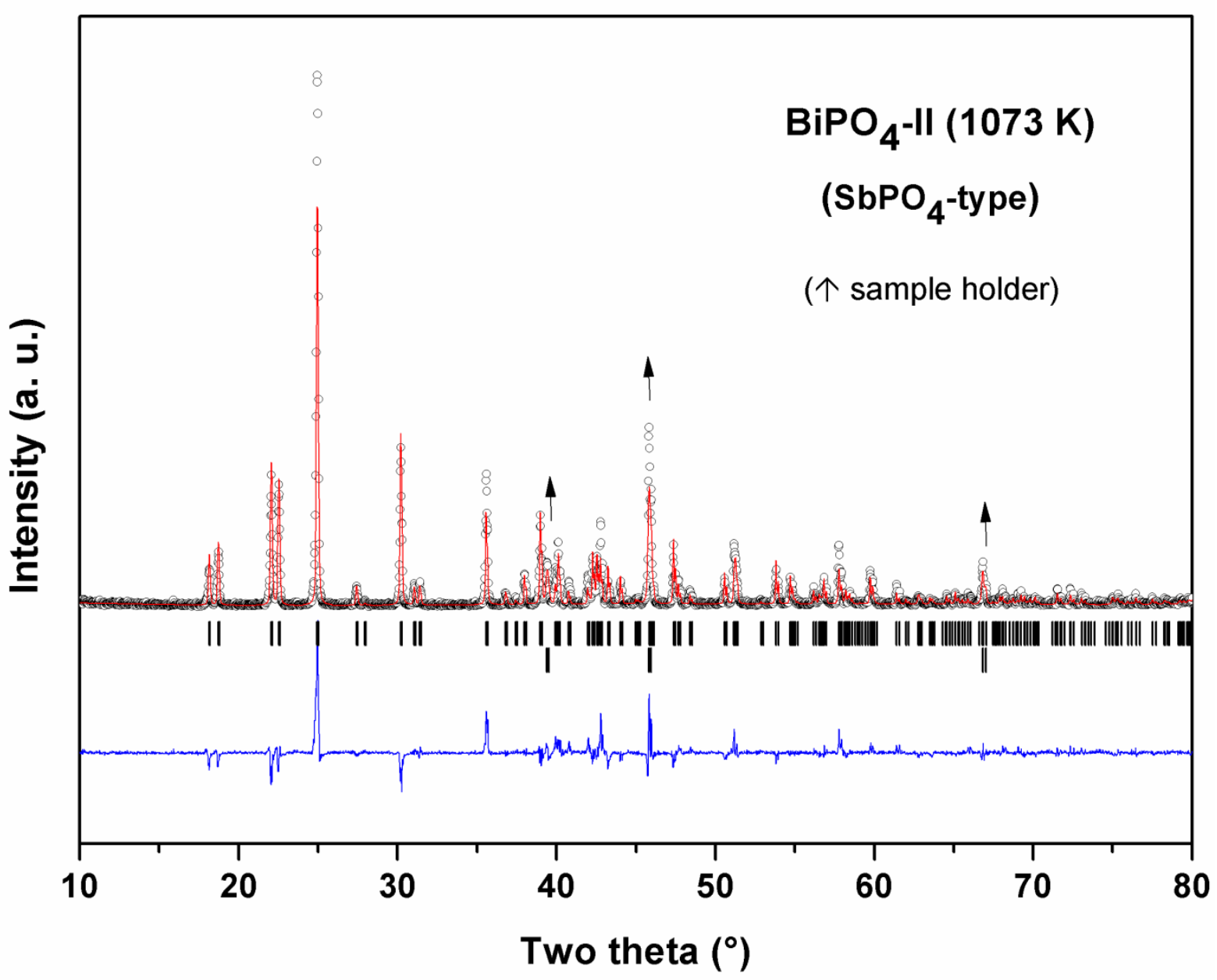

Fig.6. Rietveld refinement plot of the powder XRD data of $\mathrm{BiPO}_{4}$-II at $1073 \mathrm{~K}$. (Only $\mathrm{SbPO}_{4}$ type phase is observed). Arrows indicate reflections due to sample holder) 
Table-3. Refined structural parameters of BiPO4-III ( $\mathrm{SbPO}_{4}$ type) at different temperatures. (As observed from powder XRD data)

\begin{tabular}{|c|c|c|c|c|c|c|c|c|c|c|}
\hline & HT & 002 & 003 & 004 & 005 & 006 & 007 & 008 & 009 & 010 \\
\hline Temp & 298 & 373 & 473 & 573 & 673 & 773 & 873 & 973 & 1073 & 1173 \\
\hline a $(\AA)$ & $4.8804(1)$ & $4.8828(1)$ & $4.8857(1)$ & $4.8884(1)$ & $4.8912(1)$ & $4.8936(1)$ & $4.8959(1)$ & $4.8980(1)$ & $4.8997(1)$ & $4.9015(1)$ \\
\hline $\mathrm{b}(\AA)$ & 7.0684(2) & $7.0729(2)$ & $7.0797(2)$ & 7.0867(2) & $7.0935(2)$ & $7.1011(2)$ & 7.10897(18) & 7.1169(2) & $7.1252(2)$ & $7.1346(2)$ \\
\hline c $(\AA)$ & $4.7033(1)$ & $4.7067(1)$ & $4.7116(1)$ & $4.7170(1)$ & $4.7228(1)$ & $4.7290(1)$ & $4.73601(12)$ & $4.7434(1)$ & $4.7520(1)$ & $4.7618(1)$ \\
\hline$\beta\left(^{\circ}\right)$ & $96.285(3)$ & $96.219(3)$ & $96.126(2)$ & $96.022(2)$ & $95.915(2)$ & $95.792(2)$ & $95.662(2)$ & $95.510(2)$ & $95.342(2)$ & $95.146(2)$ \\
\hline $\mathrm{V}(\AA)^{3}$ & $161.272(8)$ & 161.591(8) & $162.039(7)$ & $162.508(7)$ & $162.985(7)$ & $163.496(7)$ & $164.032(7)$ & $164.583(7)$ & $165.177(7)$ & $165.849(7)$ \\
\hline \multirow{4}{*}{$\begin{array}{c}\text { BI } \\
\text { x y z Biso } \AA^{2}\end{array}$} & $0.1433(4)$ & $0.1437(4)$ & $0.1444(4)$ & $0.1462(4)$ & $0.1458(4)$ & $0.1477(4)$ & $0.1484(5)$ & $0.1491(5)$ & $0.1495(5)$ & $0.1496(6)$ \\
\hline & 0.2500 & 0.2500 & 0.2500 & 0.2500 & 0.2500 & 0.2500 & 0.2500 & 0.2500 & 0.2500 & 0.2500 \\
\hline & $0.1681(4)$ & $0.1673(4)$ & $0.1664(4)$ & $0.1671(4)$ & $0.1662(4)$ & $0.1658(4)$ & $0.1653(4)$ & $0.1652(5)$ & $0.1645(5)$ & $0.1648(6)$ \\
\hline & $0.33(5)$ & $0.21(5)$ & $0.28(5)$ & $0.34(5)$ & $0.57(6)$ & $0.72(6)$ & $0.93(6)$ & $1.25(6)$ & $1.60(7)$ & $1.91(8)$ \\
\hline \multirow[t]{4}{*}{$\mathrm{P}$} & $0.3751(9)$ & $0.3741(9)$ & $0.3724(9)$ & $0.3697(9)$ & $0.3717(9)$ & $0.3699(9)$ & $0.3696(9)$ & $0.3691(9)$ & $0.3689(9)$ & $0.3683(9)$ \\
\hline & 0.7500 & 0.7500 & 0.7500 & 0.7500 & 0.7500 & 0.7500 & 0.7500 & 0.7500 & 0.7500 & 0.7500 \\
\hline & $0.3092(9)$ & $0.3067(9)$ & $0.3065(9)$ & $0.3088(9)$ & $0.3090(9)$ & $0.3094(9)$ & $0.3094(9)$ & $0.3081(9)$ & $0.3082(9)$ & $0.3086(10)$ \\
\hline & 0.32 & 0.32 & 0.32 & 0.32 & 0.32 & 0.32 & 0.320 & 0.32 & $0.2(3)$ & $0.3(4)$ \\
\hline \multirow[t]{4}{*}{ O1 } & $0.676(2)$ & $0.673(2)$ & $0.674(2)$ & $0.672(2)$ & $0.672(2)$ & $0.671(2)$ & $0.671(2)$ & $0.672(2)$ & $0.670(2)$ & $0.670(2)$ \\
\hline & 0.750 & 0.7500 & 0.750 & 0.750 & 0.7500 & 0.750 & 0.750 & 0.750 & 0.750 & 0.750 \\
\hline & $0.234(4)$ & $0.225(4)$ & $0.232(4)$ & $0.238(4)$ & $0.231(4)$ & $0.235(5)$ & $0.236(5)$ & $0.238(5)$ & $0.234(5)$ & $0.235(6)$ \\
\hline & $1.0(3)$ & $0.7(3)$ & $0.8(3)$ & $1.0(3)$ & $1.0(3)$ & $1.6(4)$ & $2.2(4)$ & $2.0(4)$ & $3.1(4)$ & $4.3(5)$ \\
\hline \multirow[t]{4}{*}{$\mathrm{O} 2$} & $0.603(4)$ & $0.601(4)$ & $0.608(4)$ & $0.611(4)$ & $0.611(4)$ & $0.609(5)$ & $0.611(5)$ & $0.617(5)$ & $0.619(5)$ & $0.622(7)$ \\
\hline & 0.250 & 0.2500 & 0.2500 & 0.2500 & 0.2500 & 0.250 & 0.250 & 0.250 & 0.250 & 0.250 \\
\hline & $0.361(1)$ & $0.364(1)$ & $0.365(1)$ & $0.363(1)$ & $0.363(1)$ & 0.363(1 & $0.363(1)$ & $0.365(1)$ & $0.366(1)$ & $0.365(1)$ \\
\hline & $1.0(3)$ & $0.7(3)$ & $0.8(3)$ & $1.0(3)$ & $1.0(3)$ & $1.6(4)$ & $2.2(4)$ & $2.0(4)$ & $3.1(4)$ & $4.3(5)$ \\
\hline \multirow[t]{4}{*}{ O3 } & $0.220(3)$ & $0.220(3)$ & $0.218(3)$ & $0.217(3)$ & $0.218(3)$ & $0.212(3)$ & $0.211(3)$ & $0.212(3)$ & $0.204(3)$ & $0.202(4)$ \\
\hline & $0.578(1)$ & $0.577(1)$ & $0.577(1)$ & $0.577(1)$ & $0.577(1)$ & $0.580(1)$ & $0.578(1)$ & $0.578(1)$ & $0.581(2)$ & $0.581(2)$ \\
\hline & $0.183(3)$ & $0.184(3)$ & $0.188(3)$ & $0.188(3)$ & $0.191(3)$ & $0.186(3)$ & $0.193(3)$ & $0.194(3)$ & $0.195(4)$ & $0.199(4)$ \\
\hline & $1.0(3)$ & $0.7(3)$ & $0.8(3)$ & $1.0(3)$ & $1.0(3)$ & $1.6(4)$ & $2.2(4)$ & $2.0(4)$ & $3.1(4)$ & $4.3(5)$ \\
\hline $\begin{array}{c}\text { Rp, Rwp } \\
\chi^{2}\end{array}$ & $\begin{array}{l}13.5,19.0, \\
1.60\end{array}$ & $\begin{array}{l}\text { 13.4, 18.6, } \\
1.54\end{array}$ & $\begin{array}{l}\text { 13.2, 18.7, } \\
1.56\end{array}$ & $\begin{array}{l}\text { 13.0,18.3, } \\
1.51\end{array}$ & $\begin{array}{l}\text { 13.6, 18.7, } \\
1.58\end{array}$ & $\begin{array}{l}\text { 13.4, 18.8, } \\
1.60\end{array}$ & $\begin{array}{l}13.5,19.0, \\
1.63\end{array}$ & $\begin{array}{l}\text { 13.6, 18.8, } \\
1.61\end{array}$ & $\begin{array}{l}\text { 14.4, 19.6, } \\
1.74\end{array}$ & $\begin{array}{l}15.7,21.2, \\
1.90\end{array}$ \\
\hline $\mathrm{RB}, \mathrm{RF}$ & $5.97,3.41$ & $5.62,3.35$ & $5.98,3.53$ & $5.41,3.52$ & $5.48,3.87$ & $5.65,3.90$ & $5.43,4.32$ & $5.98,4.90$ & $6.52,5.30$ & $7.17,6.06$ \\
\hline
\end{tabular}


Table-3. Refined structural parameters of $\mathrm{BiPO}_{4}$-III ( $\mathrm{SbPO}_{4}$ type) at different temperatures. (As observed from powder XRD data)

\begin{tabular}{|c|c|c|c|c|c|c|c|c|c|c|}
\hline & HT & 002 & 003 & 004 & 005 & 006 & 007 & 008 & 009 & 010 \\
\hline $\begin{array}{ll}\mathrm{Bi}-\mathrm{O} 1 & (\AA) \\
\mathrm{Bi}-\mathrm{I} 1 & \\
\mathrm{Bi}-\mathrm{O} 2 & \\
\mathrm{Bi}-\mathrm{O} 2 & \\
\mathrm{Bi}-\mathrm{O} 3 & \\
\mathrm{Bi}-\mathrm{O} 3 & \\
\mathrm{Bi}-\mathrm{O} 3 & \\
\mathrm{Bi}-\mathrm{O} 3 & \end{array}$ & \begin{tabular}{|l|}
$2.172(18)$ \\
$2.853(18)$ \\
$2.881(18)$ \\
$2.326(18)$ \\
$2.351(9)$ \\
$2.589(13)$ \\
$2.589(13)$ \\
$2.351(9)$ \\
\end{tabular} & $\begin{array}{l}2.138(18) \\
2.903(18) \\
2.899(19) \\
2.322(18) \\
2.343(9) \\
2.598(13) \\
2.598(13) \\
2.343(9)\end{array}$ & $\begin{array}{l}2.161(18) \\
2.879(18) \\
2.874(19) \\
2.356(18) \\
2.343(9) \\
2.606(13) \\
2.606(13) \\
2.343(9)\end{array}$ & $\begin{array}{l}2.191(18) \\
2.853(18) \\
2.864(19) \\
2.361(18) \\
2.341(9) \\
2.616(13) \\
2.616(13) \\
2.341(9)\end{array}$ & $\begin{array}{l}2.160(18) \\
2.895(18) \\
2.864(19) \\
2.367(18) \\
2.345(9) \\
2.628(13) \\
2.628(13) \\
2.345(9)\end{array}$ & $\begin{array}{l}2.172(22) \\
2.883(23) \\
2.883(23) \\
2.353(22) \\
2.364(10) \\
2.595(13) \\
2.595(13) \\
2.364(10)\end{array}$ & $\begin{array}{l}2.173(22) \\
2.885(23) \\
2.878(23) \\
2.364(23) \\
2.355(10) \\
2.623(13) \\
2.623(13) \\
2.355(10)\end{array}$ & $\begin{array}{l}2.180(22) \\
2.882(23) \\
2.857(23) \\
2.394(23) \\
2.358(10) \\
2.636(13) \\
2.636(13) \\
2.358(10)\end{array}$ & \begin{tabular}{|l|}
$2.164(22)$ \\
$2.912(23)$ \\
$2.852(23)$ \\
$2.407(23)$ \\
$2.378(12)$ \\
$2.610(15)$ \\
$2.610(15)$ \\
$2.378(12))$
\end{tabular} & \begin{tabular}{|l|}
$2.170(27)$ \\
$2.915(27)$ \\
$2.837(32)$ \\
$2.424(32)$ \\
$2.382(14)$ \\
$2.623(18)$ \\
$2.623(18)$ \\
$2.382(15)$
\end{tabular} \\
\hline$<\mathrm{P}-\mathrm{O}>(\AA)$ & 2.514 & 2.518 & 2.521 & 2.523 & 2.529 & 2.526 & 2.532 & 2.538 & \begin{tabular}{|l}
2.539 \\
\end{tabular} & \begin{tabular}{|l}
2.544 \\
\end{tabular} \\
\hline $\begin{array}{ll}\text { Distt. } & \\
\text { P-O1 } & (\AA) \\
\text { P-O2 } & \\
\text { P-O3 } & \\
\text { P-O3 } & \end{array}$ & \begin{tabular}{|l|}
$92.3 \times 10^{-4}$ \\
$1.549(11)$ \\
$1.543(7)$ \\
$1.516(11)$ \\
$1.516(11)$ \\
\end{tabular} & $\begin{array}{l}108.4 \times 10^{-4} \\
1.548(12) \\
1.544(6) \\
1.517(11) \\
1.517(11)\end{array}$ & $\begin{array}{l}95.8 \times 10^{-4} \\
1.548(11) \\
1.542(7) \\
1.514(11) \\
1.514(11)\end{array}$ & $\begin{array}{l}87.5 \times 10^{-4} \\
1.548(11) \\
1.543(7) \\
1.518(11) \\
1.518(11)\end{array}$ & $\begin{array}{l}97.0 \times 10^{-4} \\
1.548(11) \\
1.544(7) \\
1.517(11) \\
1.517(11)\end{array}$ & $\begin{array}{l}92.4 \times 10^{-4} \\
1.549(12) \\
1.544(7) \\
1.519(12) \\
1.519(12)\end{array}$ & $\begin{array}{l}93.8 \times 10^{-4} \\
1.551(12) \\
1.545(7) \\
1.521(12) \\
1.521(12)\end{array}$ & $\begin{array}{l}88.0 \times 10^{-4} \\
1.550(12) \\
1.547(7) \\
1.517(12) \\
1.517(12)\end{array}$ & $\begin{array}{l}88.7 \times 10^{-4} \\
1.548(13) \\
1.546(7) \\
1.519(13) \\
1.519(13)\end{array}$ & $\begin{array}{l}85.3 \times 10^{-4} \\
1.550(14) \\
1.549(7) \\
1.519(16) \\
1.519(16)\end{array}$ \\
\hline
\end{tabular}




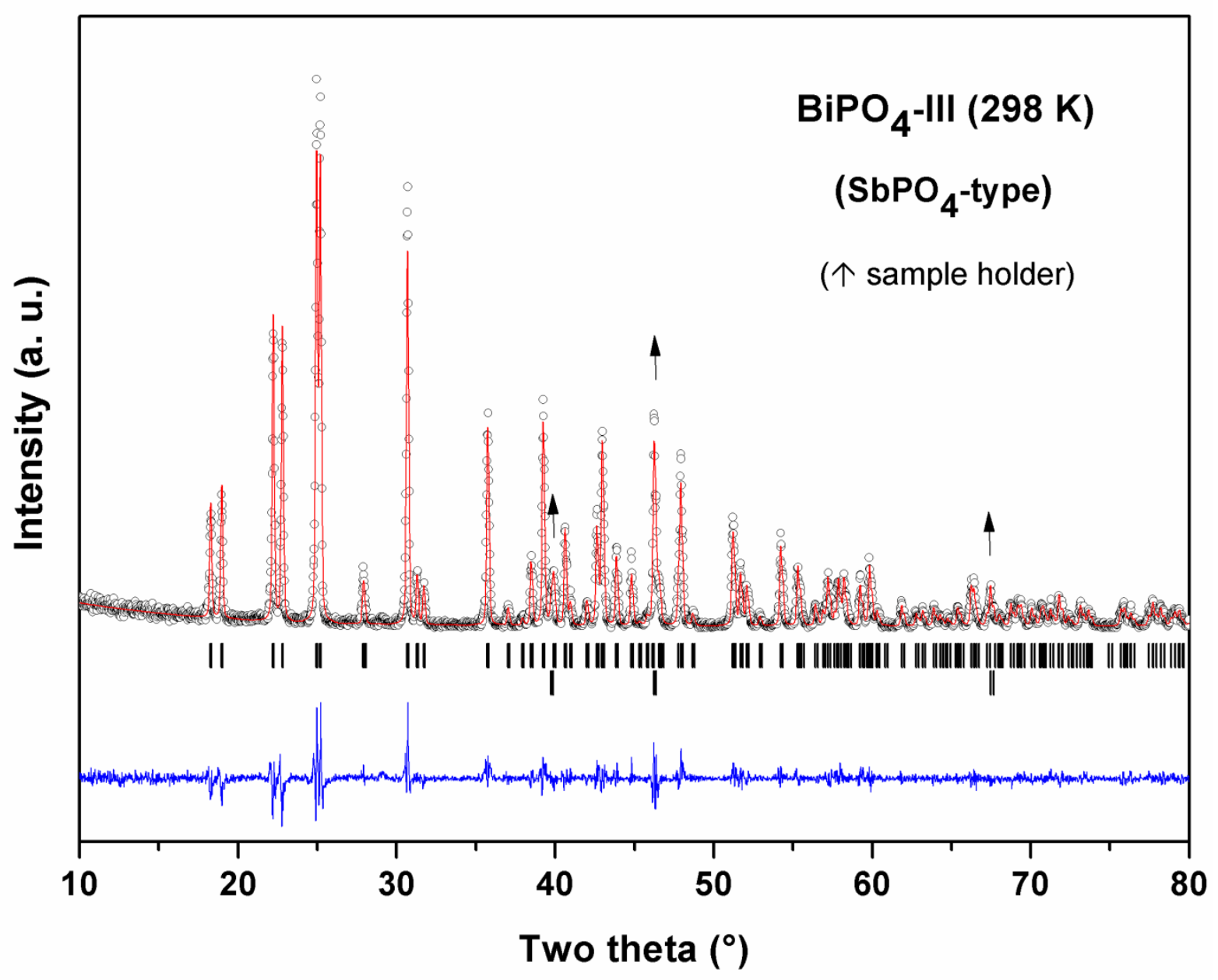

Fig.7. Rietveld refinement plot of the powder XRD data of $\mathrm{BiPO}_{4}-\mathrm{III}$ at $298 \mathrm{~K}$. (Only $\mathrm{SbPO}_{4^{-}}$ type phases is observed). Arrows indicate reflections due to sample holder) 


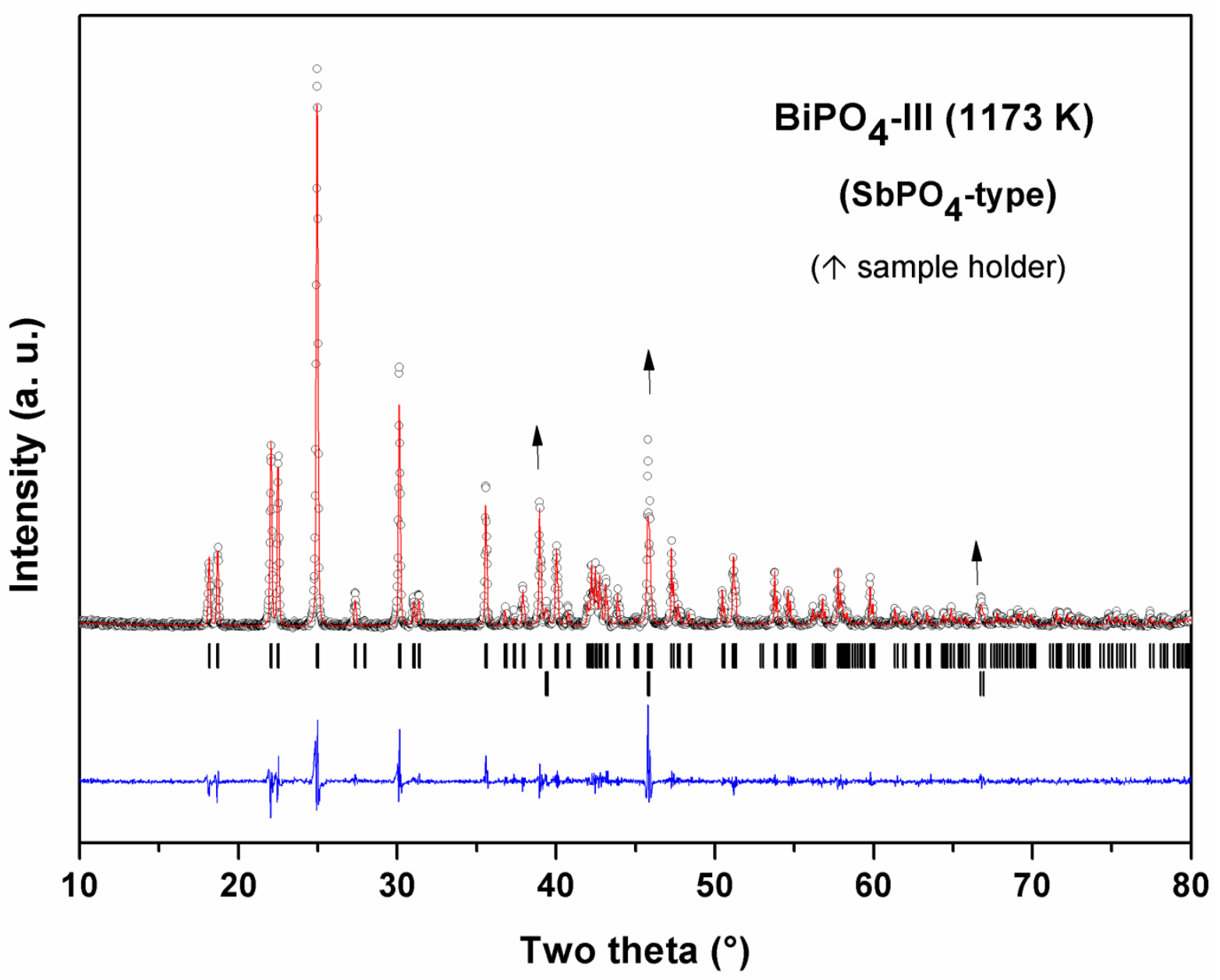

Fig.8. Rietveld refinement plot of the powder XRD data of $\mathrm{BiPO}_{4}$-III at $1173 \mathrm{~K}$. (Only $\mathrm{SbPO}_{4}$-type phase is observed). Arrows indicate reflections due to sample holder) 


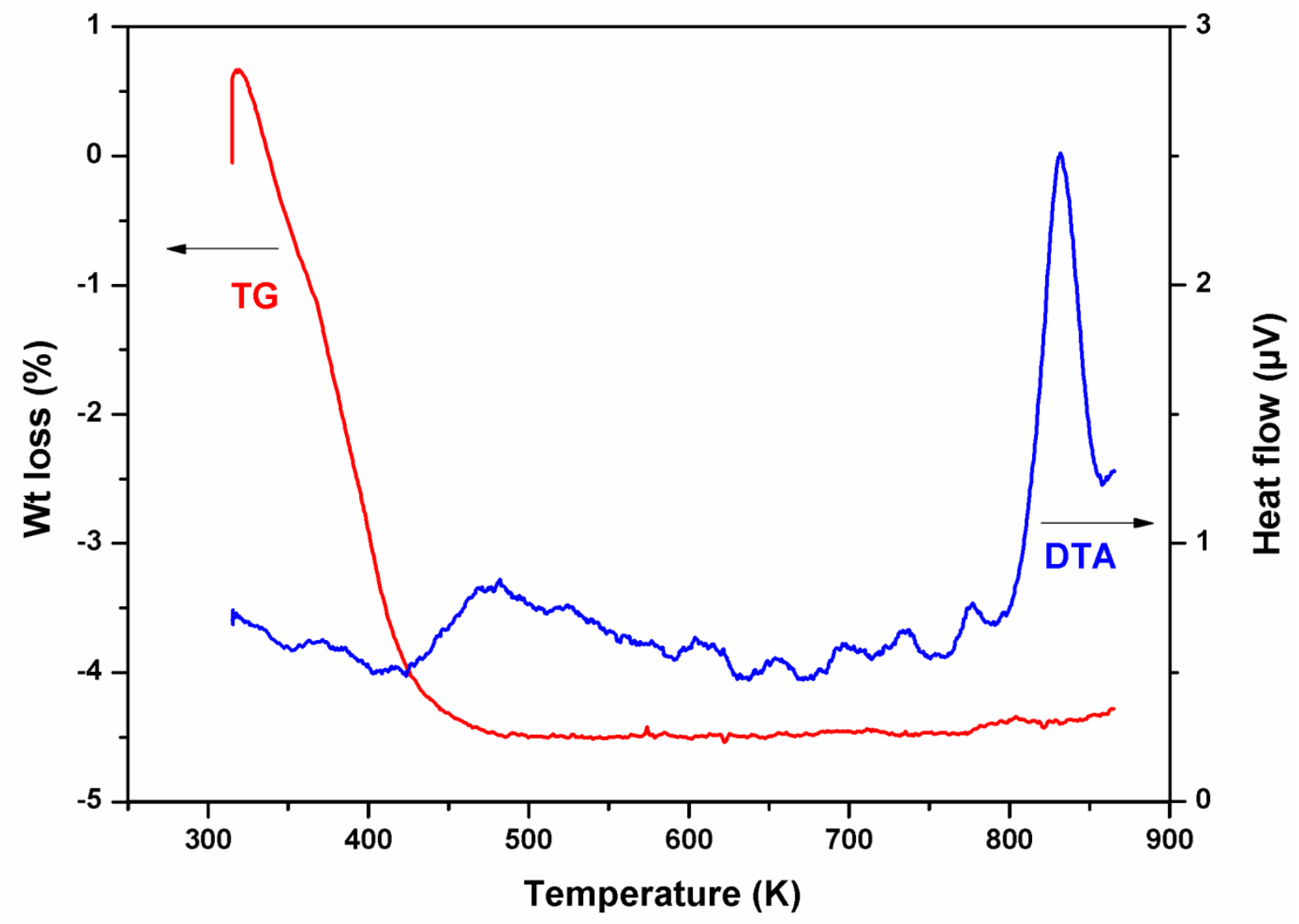

Fig.9. Typical TG-DTA traces of as prepared sample of hydrated trigonal $\mathrm{BiPO}_{4}$ sample. (Sample was heated from 323 to $873 \mathrm{~K}$ at a rate of $10 \mathrm{~K} / \mathrm{min}$ ). 